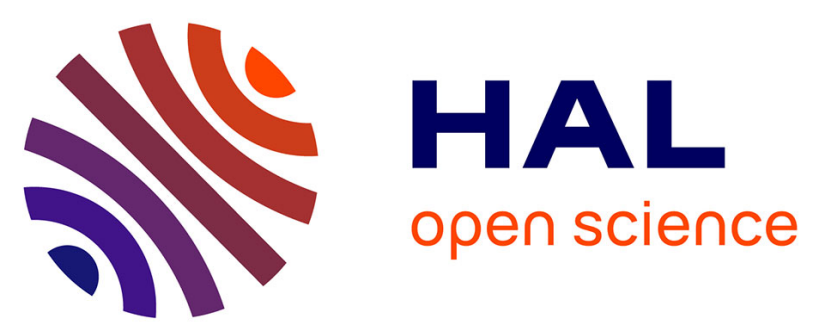

\title{
Nickel complexes of 1,2,4-triazole derived amido-functionalized N-heterocyclic carbene ligands: Synthesis, theoretical studies and catalytic application
} Anuj Kumar, Linus Paulin Bheeter, Manoj Kumar Gangwar, Jean-Baptiste Sortais, Christophe Darcel, Prasenjit Ghosh

\section{To cite this version:}

Anuj Kumar, Linus Paulin Bheeter, Manoj Kumar Gangwar, Jean-Baptiste Sortais, Christophe Darcel, et al.. Nickel complexes of 1,2,4-triazole derived amido-functionalized N-heterocyclic carbene ligands: Synthesis, theoretical studies and catalytic application. Journal of Organometallic Chemistry, 2015, 786, pp.63-70. 10.1016/j.jorganchem.2015.03.007 . hal-01224508

HAL Id: hal-01224508

https://hal-univ-rennes1.archives-ouvertes.fr/hal-01224508

Submitted on 28 Jan 2016

HAL is a multi-disciplinary open access archive for the deposit and dissemination of scientific research documents, whether they are published or not. The documents may come from teaching and research institutions in France or abroad, or from public or private research centers.
L'archive ouverte pluridisciplinaire HAL, est destinée au dépôt et à la diffusion de documents scientifiques de niveau recherche, publiés ou non, émanant des établissements d'enseignement et de recherche français ou étrangers, des laboratoires publics ou privés. 
Nickel Complexes of 1,2,4-Triazole Derived Amido-functionalized Nheterocyclic Carbene Ligands: Synthesis, Theoretical Studies and Catalytic Application

Anuj Kumar, ${ }^{\dagger}$ Linus Paulin Bheeter, ${ }^{\star}$ Manoj Kumar Gangwar, ${ }^{\dagger}$ Jean-Baptiste Sortais, ${ }^{\ddagger}$ Christophe Darcel*,+் and Prasenjit Ghosh*, ${ }^{*}$

${ }^{\dagger}$ Department of Chemistry

Indian Institute of Technology Bombay, Powai, Mumbai 400076.

†Université de Rennes 1,

UMR CNRS-UR1 6226 Institut des Sciences chimiques de Rennes, Team "Organometallics: Materials and Catalysis" - Centre for Catalysis and Green Chemistry

Campus de Beaulieu, 35042 - Rennes, France

Email:pghosh@chem.iitb.ac.in, christophe.darcel@univ-rennes1.fr, Fax: +91222572 3480, +33223236939 
Keywords: nickel; $N$-heterocyclic carbene; borylation; aryl bromide; DFT studies 
Abstract: A series of nickel complexes $(\mathbf{1}-\mathbf{3}) \mathbf{b}$ of 1,2,4-triazole derived amidofunctionalized $\mathrm{N}$-heterocyclic carbene ligands were synthesized and structurally characterized. In particular, the [1-(R)-4- $N$-(furan-2-ylmethyl)acetamido-1,2,4triazol-5-ylidene ${ }_{2} \mathrm{Ni}[\mathrm{R}=\mathrm{Et}(\mathbf{1 b}), i-\operatorname{Pr}(\mathbf{2 b})$ and $\mathrm{Bn}(\mathbf{3 b})]$ complexes were obtained by the direct reaction of the corresponding triazolium chloride salts $(\mathbf{1}-\mathbf{3}) \mathbf{a}$ by the treatment with $\mathrm{NiCl}_{2} \cdot 6 \mathrm{H}_{2} \mathrm{O}$ in presence of $\mathrm{K}_{2} \mathrm{CO}_{3}$ as a base. The density functional theory studies performed on these complexes reveal highly polar character of the NHC-Ni $\sigma$-bonding interaction with corresponding molecular orbital having a maximum contribution (59-69\%) from the NHC ligand fragments while that of a minimum contribution (4\%) from the central nickel atom. The (1-3)b complexes were found to be moderately active for the catalytic borylation reactions of bromoaryl derivatives by bis(pinacolato)diboron reagent $\left(\mathrm{B}_{2} \mathrm{pin}_{2}\right)$ in the presence of $\mathrm{Cs}_{2} \mathrm{CO}_{3}$ as a base at $70{ }^{\circ} \mathrm{C}$. 


\section{Introduction}

Recent increase in interest in nickel catalysis has led to the discovery of many interesting catalytic transformations and has thrown open exciting possibilities for this metal [1]. In comparison to its immediate congener, palladium, [2] that has seen phenomenal success in catalysis in recent times, nickel has surprisingly maintained a low profile all throughout. As a matter of fact, nickel, as a metal, is quite attractive for catalysis mainly for its high nucleophilicity that arise out of its small size as a $1^{\text {st }}$ row transition metal, and for its several accessible oxidation states, [3] that make it flexible towards various catalytic cycles [4]. In addition to these favorable catalytic attributes, nickel is inexpensive and thus, as a whole, is ideally suited for catalysis.

One of our interests [5] lies on exploring the catalytic potential of nickel, and towards this goal, we intended to use the now popular $N$-heterocyclic carbene ligands that are widely recognized for their success in homogeneous catalysis [6]. In this context, we have recently observed the superiority of the nickel $N$-heterocyclic carbene complexes over their palladium analogs in the hydroamination reactions of the activated olefins [7]. We have also designed nickel $N$-heterocyclic carbene complexes for the bifunctional catalysis of the Michael addition reaction under base-free conditions by employing suitably tailored chelating amido-functionalized $\mathrm{N}$-heterocyclic carbene ligands of the imidazole scaffold [8,9]. Meanwhile, some of us were also interested in the reactivity of half-sandwich $N$-heterocyclic carbene (NHC) nickel complexes in efficient and chemoselective catalytic hydrosilylation of aldehydes, ketones and imines under mild conditions [10]. 
Extending even further, we sought to explore the utility of the nickel complexes of the related amido-functionalized $N$-heterocyclic carbene ligands of the 1,2,4-triazole scaffold in various other catalytic transformations like in the catalytic borylation reactions of bromoaryl derivatives by bis(pinacolato)diboron. The borylation reaction [11] is important for providing convenient access to the high value intermediates of the day-to-day organic synthesis, mainly for the Suzuki-Miyaura Cross-Coupling reaction. In this context it is noteworthy that the nickel catalysts, despite being among the pioneering ones for the $\mathrm{C}-\mathrm{C}$ cross coupling reaction, as observed from the early report of the Corriu-Kumada coupling with Grignard reagents [12], they remained less popular till date than their palladium counterparts in various $\mathrm{C}-\mathrm{C}$ cross coupling reactions including the Suzuki-Miyaura reaction [13 ]. From this perspective, quite importantly, the recent reports of the nickel catalysis for the borylation reactions of the organohalide derivatives providing convenient access to the borylated synthons [14], would ignite interest on the metal. In addition to the nickel, our other interest in the 1,2,4-triazole based $N$-heterocyclic carbene ligands arises from the fact that these ligands are less explored as compared to their imidazole analogues $[15,16]$.

Hence, in this contribution, we report a series of nickel complexes namely [1-(R)-4$N$-(furan-2-ylmethyl)acetamido-1,2,4-triazol-5-ylidene $]_{2} \mathrm{Ni}[\mathrm{R}=\mathrm{Et}(\mathbf{1 b}), i$-Pr (2b) and $\mathrm{Bn}(\mathbf{3 b})]$ of the 1,2,4-triazole derived $N$-heterocyclic carbene ligands that show low to moderate activity in the catalytic borylation reactions of bromoaryl derivatives by bis(pinacolato)diboron. 


\section{Results and Discussions}

A series of amido-functionalized $N$-heterocyclic carbene precursors namely, 1-(R)-4$N$-(furan-2-ylmethyl)acetamido-1,2,4-triazolium chloride [R = Et (1a), $i$-Pr (2a), Bn (3a)] were synthesized from the respective 1-(R)-1,2,4-triazoles by the direct reaction of 2-chloro- $N$-(furan-2-ylmethyl)acetamide in 52-66 \% yield (Scheme 1). The formation of the 1,2,4-triazolium chloride salts (1-3)a was very much evident from the appearances of the characteristic $(\mathrm{N} \underline{C H} \mathrm{~N})$ resonances at a highly downfield region at 10.8-10.2 ppm in the ${ }^{1} \mathrm{H}$ NMR spectrum and at $146.1-145.8 \mathrm{ppm}$ in the ${ }^{13} \mathrm{C}\left\{{ }^{1} \mathrm{H}\right\}$ NMR spectrum and were further confirmed by High Resolution Mass Spectrometry (HRMS) studies that showed the $[\mathrm{NHC}+\mathrm{H}]^{+}$peaks at $\mathrm{m} / \mathrm{z} 235.1206$ (1a), $\mathrm{m} / \mathrm{z}$ 249.1356 (2a) and $m / z 297.1350$ (3a) against the corresponding calculated values of $m / z 235.1195$ (1a), $m / z 249.1352(\mathbf{2 a})$ and $m / z 297.1352$ (3a).

The nickel complexes $(\mathbf{1}-\mathbf{3}) \mathbf{b}$ were then synthesized from the respective triazolium chloride salts (1-3)a by the treatment with $\mathrm{NiCl}_{2} \cdot 6 \mathrm{H}_{2} \mathrm{O}$ in $\mathrm{CH}_{3} \mathrm{CN}$ in presence of $\mathrm{K}_{2} \mathrm{CO}_{3}$ (4.4 equiv.) as a base (Scheme 1 and Figure 1). The diamagnetic nature of the (1-3)b complexes indicated a square planar geometry at the metal center in these complexes. The ${ }^{1} \mathrm{H}$ NMR spectrum showed the absence of the $\mathrm{NC} \underline{H} \mathrm{~N}$ and amido-N $\underline{H}$ resonances, consistent with the formation of a carbene center on the triazolium ring along with the chelation of the anionic amido-functionalized side arm to the metal center in these complexes. Quite interestingly, the two methylene moieties of the (1-2)b complexes were diastereotopic in nature and exhibited four sets of resonances for the $\mathbf{1 b}$ and the $\mathbf{2} \mathbf{b}$ complexes while displaying a ${ }^{2} J_{\mathrm{HH}}$ coupling constant of $15 \mathrm{~Hz}$. In contrast, all of the three methylene groups of the $\mathbf{3 b}$ complex were also 
diastereotopic in nature displaying six sets of resonances while exhibiting a ${ }^{2} J_{\mathrm{HH}}$ coupling constant of $15 \mathrm{~Hz}$ in the ${ }^{1} \mathrm{H}$ NMR spectrum. Lastly, the characteristic $\mathrm{Ni}-\mathrm{C}_{\text {carbene }}$ resonance appeared at $171.6-171.0 \mathrm{ppm}$ region in ${ }^{13} \mathrm{C}\left\{{ }^{1} \mathrm{H}\right\} \mathrm{NMR}$ spectrum of the $(\mathbf{1}-\mathbf{3}) \mathbf{b}$ complexes.

The single crystals of (1-3)b were duly obtained from an acetonitrile solution for the X-ray single crystal diffraction analysis. The molecular structure of the (1-3)b complexes determined by the X-ray diffraction studies showed the metal center in a square-planar geometry (Figure 2-4 and Table 1). The nickel in the (1-3)b complexes is linked to two chelated $N$-heterocyclic carbene ligands, which were arranged in a cis-disposition to each other and were bound to the metal center through the carbene- $\mathrm{C}$ and an amido- $\mathrm{N}$ atoms. The $\mathrm{Ni}-\mathrm{C}_{\text {carbene }}$ bond distances in the $\mathbf{1 b}$ [1.860(3) $\AA$ and 1.859(3) $\AA], 2 \mathbf{b}[1.853(2) \AA$ and 1.853(2) $\AA]$ and 3b [1.871(4) $\AA$ and 1.871(4) $\AA$ ] complexes compare well with the average $d\left(\mathrm{Ni}-\mathrm{C}_{\text {carbene }}\right)$ distance $[1.864$ $\AA]$ observed for the related structurally characterized nickel(II) complexes of chelated amido-functionalized $N$-heterocyclic carbene ligands that are present in the Cambridge Structural Database [17]. Similarly, the Ni- $\mathrm{N}_{\text {amido }}$ bond distances in $\mathbf{1 b}$

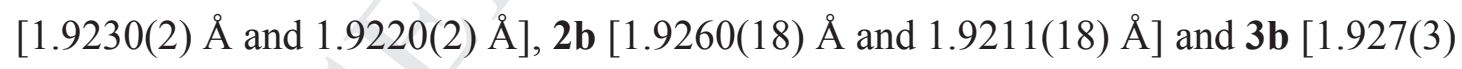
$\AA$ and $1.931(3) \AA]$ are also comparable to the average $d\left(\mathrm{Ni}-\mathrm{N}_{\text {amido }}\right)\left[\begin{array}{ll}1.932 \AA\end{array}\right]$ observed for the related structurally characterized complexes in the Cambridge Structural Database [17].

Further, insights into the electronic structures of these (1-3)b complexes were obtained with the aid of the density functional theory (DFT) study. Specifically, the geometry optimized structures were computed for the (1-3)b complexes at the 
B3LYP/LANL2DZ, 6-31G(d) level of theory by using the atomic coordinates obtained from the X-ray diffraction studies (Supporting Information Figure S34-S36 and Table S7-S9). The computed structures are in good agreement with the experimental ones (Supporting Information Table S10-S12). Subsequently, the single-point calculations along with the post-wave function analysis using the natural bond order (NBO) method were performed on the geometry optimized structures at the same level of theory for a detailed understanding of the electronic properties of these complexes.

Corroborating the view of a strong electron-donation from the anionic amidofunctionalized $N$-heterocyclic carbene (NHC) ligands to the metal center in the (1-3)b complexes, the natural and the Mulliken charge analyses indeed showed significant increase in the electron density at the nickel center in the $(\mathbf{1}-\mathbf{3}) \mathbf{b}$ complexes relative to that of the free $\mathrm{Ni}^{2+}$ ion (Supporting Information Table S1-S3). Along the same line, a scrutiny of the electronic configuration of the metal center in the (1-3)b complexes further revealed that the electron donation from the carbene lone pair occurred onto the $3 d$ and $4 s$ orbitals of the nickel center (Supporting Information Table S4).

Of particular interest are the $\mathrm{Ni}-\mathrm{C}_{\text {carbene }}$ bonding interaction in the $(\mathbf{1}-\mathbf{3}) \mathbf{b}$ complexes. The NBO analysis showed that the $\mathrm{Ni}-\mathrm{C}_{\text {carbene }}$ bonds in the $(\mathbf{1}-\mathbf{3}) \mathbf{b}$ complexes comprised of an interaction between a $s p^{2}$ hybridized $\mathrm{C}_{\text {carbene }}$ orbital with a $d s p^{2}$ hybridized Ni orbital, and this is in agreement with the square-planar geometry of the central metal atom (Supporting Information Table S5). Additional understanding of the $\mathrm{Ni}-\mathrm{C}_{\text {carbene }}$ interaction were obtained by the Charge Decomposition Analysis (CDA) that estimated the NHC ligand to the nickel $\sigma$-donation, as denoted by $d$, and 
the nickel to the NHC ligand $\pi$-back-donation, as denoted by $b$, occurring in these (1-3)b complexes. As expected of the anionic amido-functionalized $N$-heterocyclic carbene ligands in the $(\mathbf{1}-\mathbf{3}) \mathbf{b}$ complexes to be strongly $\sigma$-donating, very high $d / b$ ratio of $10.95(\mathbf{1 b}), 11.30(\mathbf{2 b})$, and $11.25(\mathbf{3 b})$ were observed in these complexes (Supporting Information Table S6). It is worth noting that the forward $\sigma$-donation $(d)$ and the backward $\pi$ - donation $(b)$ values of the $(\mathbf{1}-\mathbf{3}) \mathbf{b}$ complexes represent the interactions between the metal center and the two amido functionalized NHC-ligand fragments, and which individually is composed of two interactions namely, a $\mathrm{C}_{\text {carbene }}{ }^{-}$ $\mathrm{Ni}$ interaction and a $\mathrm{N}_{\text {amido }}-\mathrm{Ni}$ interaction.

The molecular orbital (MO) correlation diagrams were constructed for the $(\mathbf{1}-\mathbf{3}) \mathbf{b}$ complexes from the interaction of the individual fragment molecular orbitals (FMOs) of the free NHC ligand fragments with that of the free $\mathrm{Ni}^{2+}$ ion. The molecular orbital (MO) representing the $\mathrm{NHC}-\mathrm{Ni} \sigma$-interactions in the $(\mathbf{1}-\mathbf{3}) \mathbf{b}$ complexes i.e. the HOMO-36 (1b), HOMO-43 (2b) and the HOMO-42 (3b) is formed from the interaction between the carbene lone pairs of the two NHC ligand fragments with the $4 s$ atomic orbital of the $\mathrm{Ni}^{2+}$ ion (Figure 5 and Supporting Information Figure $\mathrm{S} 37$ and S38). The NHC-Ni $\sigma$-bonding molecular orbital in the (1-3)b complexes are primarily ligand based exhibiting significant contribution [69\% (1b), $69 \%$ (2b) and $59 \%(\mathbf{3 b})$ ] from the two NHC ligand fragments and marginal contribution $[4 \%(\mathbf{1 b})$, $4 \%(\mathbf{2 b})$ and $4 \%(3 \mathbf{b})]$ from the central nickel atom and this point towards a substantial ionic character of the $\mathrm{NHC}-\mathrm{Ni} \sigma$-interaction. Additionally, these NHC-Ni $\sigma$-bonding molecular orbitals namely, the HOMO-36 (1b), HOMO-43 (2b) and the HOMO-42 (3b) were all found to be deeply buried thereby suggesting highly stable nature of these $\mathrm{Ni}-\mathrm{NHC} \sigma$-interactions and further indicating that these 
$\mathrm{Ni}-\mathrm{NHC} \sigma$-bonds in the $(\mathbf{1}-\mathbf{3}) \mathbf{b}$ complexes are less susceptible to the attacks by incoming nucleophiles and electrophiles.

The recent interest on nickel as catalysts in borylation of halogeno derivatives leading to the corresponding borylated compounds useful in Suzuki-Miyaura cross-coupling reactions prompted us to examine $(\mathbf{1}-\mathbf{3}) \mathbf{b}$ as potential catalysts for the coupling of bis(pinacolato)diboron with aryl bromides. In particular, in the presence of the 3 equiv. of $\mathrm{Cs}_{2} \mathrm{CO}_{3}$, the nickel complexes $(\mathbf{1}-\mathbf{3}) \mathbf{b}$ catalyzed the reaction between the bis(pinacolato)diboron reagent $\left(\mathrm{B}_{2} \mathrm{pin}_{2}\right)(1.5$ equiv.) with the 1 equiv. of arylbromide substrates exhibiting moderate conversions (61-90\%) and yielding the corresponding borylated compounds in $13 \%, 10 \%$ and $8 \%$, respectively along with the dehalogenated arenes (Table 2, entries 1-3). It must be noticed that except the formation of the borylated derivatives, and the unreacted starting reagents, only dehalogenated arenes were obtained as side products. The formation of the reduced arene derivatives can be explained by the oxidative addition of the haloarene leading to an $\mathrm{Ni}(\mathrm{Ar})(\mathrm{X})$ intermediate which was then hydrolyzed.

Subsequently, the most active catalyst, $\mathbf{1 b}$, was used in the borylation reactions of various other arylbromide substrates. (Table 2, Entries 4-9) The ortho- and the metatolylbromide displayed $88 \%$ and $91 \%$ conversions respectively along with low amount of borylated tolyl derivatives of $12 \%$ and $14 \%$ respectively (Table 2, Entries 4 and 5). However, with the $p$-anisylbromide and p-tert-butylphenylbromide substrates, the better yields (20\% and 18\%, respectively) were observed at $73 \%$ and $71 \%$ conversions (Table 2, Entries 6 and 7). Notably, the electron-donating substituted aryl bromide substrates seem to be more reactive than the phenylbromide 
substrate ( $83 \%$ conv., $10 \%$ yield, entry 8$)$. For the 2-naphtylbromide substrate, the formation of the $20 \%$ of the corresponding borylated arene was observed (Table 2, entry 9).

\section{Conclusion}

In summary, a series of nickel complexes (1-3)b of the chelating anionic amidofunctionalized N-heterocyclic carbene ligands have been synthesized and structurally characterized. The density functional theory studies performed on the (1-3)b complexes point toward highly stable $\mathrm{NHC}-\mathrm{Ni}$ interactions that are predominantly $\sigma$ bonding in nature. The (1-3)b complexes were found to be moderately active for the catalytic borylation reactions of bromoaryl derivatives by the bis(pinacolato)diboron reagent $\left(\mathrm{B}_{2} \mathrm{pin}_{2}\right)$ in the presence of $\mathrm{Cs}_{2} \mathrm{CO}_{3}$ as a stoichiometric base at $70{ }^{\circ} \mathrm{C}$, mainly due to a competitive dehalogenation reaction. 


\section{Experimental Section}

General Procedures. All manipulations were carried out using a combination of a glovebox and standard Schlenk techniques. $\mathrm{NiCl}_{2} \bullet 6 \mathrm{H}_{2} \mathrm{O}$ was purchased from SD-fine Chemicals (India) and used without any further purification. 1-(ethyl)-1,2,4-triazole [18], 1-(i-propyl)-1,2,4-triazole [19], 1-(benzyl)-1,2,4-triazole [20] and 2-chloro-N(furan-2-ylmethyl)acetamide[21] were synthesized according to modified literature procedures. ${ }^{1} \mathrm{H}$ and ${ }^{13} \mathrm{C}\left\{{ }^{1} \mathrm{H}\right\}$ NMR spectra were recorded on a Bruker $400 \mathrm{MHz}$ NMR spectrometer. ${ }^{1} \mathrm{H}$ NMR peaks are labeled as singlet (s), doublet (d), triplet (t), quartet (q), quartet of doublets (qd), doublet of doublets (dd) septet (sept) and broad (br). Infrared spectra were recorded on a Perkin Elmer Spectrum One FT-IR spectrometer. Mass spectrometry measurements were done on a Micromass Q-Tof spectrometer and Bruker maxis impact spectrometer. Elemental Analysis was carried out on Thermo Finnigan FLASH EA 1112 SERIES (CHNS) Elemental Analyzer. Xray diffraction data for compounds $\mathbf{1 b}, \mathbf{2} \mathbf{b}$ and $\mathbf{3 b}$ were collected on an Oxford Diffraction XCALIBUR-S diffractometer and Rigaku $\mathrm{Hg} 724+$ diffractometer. Crystal data collection and refinement parameters are summarized in Table 1. The structures were solved using direct method and standard difference map techniques, and refined by full-matrix least-squares procedures on $F^{2}$. CCDC-855783 (for $\mathbf{1 b}$ ), CCDC-844351 (for 2b) and CCDC-1003751 (for 3b) contain the supplementary crystallographic data for this paper. These data can be obtained free of charge from the Cambridge Crystallographic Data center via www.ccdc.cam.ac.uk/data_request/cif. 
Synthesis of 1-(ethyl)-4-N-(furan-2-ylmethyl)acetamido-1,2,4-triazolium chloride (1a)

A mixture of ethyl triazole (1.25 g, $12.9 \mathrm{mmol})$ and 2-chloro-N-(furan-2ylmethyl)acetamide $(2.21 \mathrm{~g}, 12.8 \mathrm{mmol})$ was refluxed in toluene $(c a .40 \mathrm{~mL})$ for 16 hours during which a black viscous precipitate was formed. After decanting off the solvent, the precipitate was washed with hot petroleum ether $(c a .3 \times 10 \mathrm{~mL})$ and then dissolved in minimum amount of dichloromethane $(c a .4 \mathrm{~mL})$. The product was precipitated out by addition of diethyl ether $(c a .15 \mathrm{~mL})$. The product was isolated by decantation and was vacuum dried to give the product (1a) as a hygroscopic black solid (2.29 g, $66 \%) .{ }^{1} \mathrm{H}$ NMR $\left(\mathrm{CDCl}_{3}, 400 \mathrm{MHz}, 25^{\circ} \mathrm{C}\right): \delta 10.8(\mathrm{~s}, 1 \mathrm{H}, \mathrm{N}-\mathrm{C}(5) \underline{H}-\mathrm{N})$, 9.40 (br, $1 \mathrm{H}, \mathrm{N} \underline{H C O}), 9.39$ (s, $1 \mathrm{H}, \mathrm{N}-\mathrm{C}(3) \underline{H}-\mathrm{N}), 7.28$ (br, $\left.1 \mathrm{H}, \mathrm{C}_{4} \underline{H}_{3} \mathrm{O}\right), 6.26$ (br, $1 \mathrm{H}$, $\left.\mathrm{C}_{4} \underline{H}_{3} \mathrm{O}\right), 6.23\left(\mathrm{br}, 1 \mathrm{H}, \mathrm{C}_{4} \underline{H}_{3} \mathrm{O}\right), 5.54\left(\mathrm{~s}, 2 \mathrm{H}, \mathrm{C}_{2}\right), 4.45$ (q, $\left.2 \mathrm{H},{ }^{3} J_{\mathrm{HH}}=7 \mathrm{~Hz}, \mathrm{C}_{2} \mathrm{CH}_{3}\right)$, $4.37\left(\mathrm{~d}, 2 \mathrm{H},{ }^{3} \mathrm{~J}_{\mathrm{HH}}=5 \mathrm{~Hz}, \mathrm{C} \underline{H}_{2} \mathrm{NH}\right), 1.57\left(\mathrm{t}, 3 \mathrm{H},{ }^{3} J_{\mathrm{HH}}=7 \mathrm{~Hz}, \mathrm{CH}_{2} \mathrm{C}_{3}\right) . \quad{ }^{13} \mathrm{C}\left\{{ }^{1} \mathrm{H}\right\}$ NMR (DMSO- $\left.d_{6}, 100 \mathrm{MHz}, 25{ }^{\circ} \mathrm{C}\right): \delta 164.5(\underline{C}=\mathrm{O}), 151.4\left(\underline{C}_{4} \mathrm{H}_{3} \mathrm{O}\right), 145.8(\mathrm{~N}-\underline{C}(5)-$ $\mathrm{N}), 143.2(\mathrm{~N}-\underline{C}(3)-\mathrm{N}), 142.7\left(\underline{C}_{4} \mathrm{H}_{3} \mathrm{O}\right), 110.9\left(\underline{C}_{4} \mathrm{H}_{3} \mathrm{O}\right), 107.9\left(\underline{C}_{4} \mathrm{H}_{3} \mathrm{O}\right), 49.0\left(\underline{C}_{2}\right)$, $47.5\left(\underline{C}_{2} \mathrm{CH}_{3}\right), 36.1\left(\underline{C H}_{2}\right), 13.9\left(\mathrm{CH}_{2} \underline{C} \mathrm{H}_{3}\right) . \quad$ IR data $(\mathrm{KBr}$ pellet $) \mathrm{cm}^{-1}: 1686(\mathrm{~s})$ $\left(v_{\mathrm{c}=\mathrm{o}}\right)$. HRMS (ES): $m / z$ 235.1206 $[\mathrm{NHC}+\mathrm{H}]^{+}$, calcd. 235.1195. Anal. Calcd. for $\mathrm{C}_{11} \mathrm{H}_{15} \mathrm{~N}_{4} \mathrm{O}_{2} \mathrm{Cl} \bullet \mathrm{H}_{2} \mathrm{O}: \mathrm{C}, 45.76 ; \mathrm{H}, 5.93 ; \mathrm{N}, 19.40$. Found: $\mathrm{C}, 46.58 ; \mathrm{H}, 6.13 ; \mathrm{N}, 19.42$ $\%$.

Synthesis of [1-(ethyl)-4-N-(furan-2-ylmethyl)acetamido-1,2,4-triazol-5ylidene $]_{2} \mathrm{Ni}(1 \mathrm{~b})$

A mixture of 1-ethyl-4-N-(furan-2-ylmethyl)acetamido-1,2,4-triazolium chloride (1a) (0.640 g, $2.37 \mathrm{mmol}), \mathrm{K}_{2} \mathrm{CO}_{3}(1.64 \mathrm{~g}, 11.8 \mathrm{mmol})$ and $\mathrm{NiCl}_{2} \bullet 6 \mathrm{H}_{2} \mathrm{O}(0.280 \mathrm{~g}, 1.18$ mmol) was refluxed in acetonitrile $(c a .40 \mathrm{~mL})$ for 24 hours. The reaction mixture 
was filtered and filtrate was reduced in vaccum up to $10 \mathrm{~mL}$. The filtrate was kept at room temperature for overnight, during which yellow crystals were formed. Finally, the crystals were separated by filtration and vacuum dried to give the product (1b) as a yellow crystalline solid $(0.135 \mathrm{~g}, 22 \%) .{ }^{1} \mathrm{H} \mathrm{NMR}\left(\mathrm{CDCl}_{3}, 400 \mathrm{MHz}, 25{ }^{\circ} \mathrm{C}\right): \delta 8.02$ $(\mathrm{s}, 2 \mathrm{H}, \mathrm{N}-\mathrm{C}(3) \underline{H}-\mathrm{N}), 7.31\left(\mathrm{dd}, 2 \mathrm{H},{ }^{3} J_{\mathrm{HH}}=2 \mathrm{~Hz},{ }^{4} J_{\mathrm{HH}}=1 \mathrm{~Hz}, \mathrm{C}_{4} \underline{H}_{3} \mathrm{O}\right), 6.34(\mathrm{dd}, 2 \mathrm{H}$, $\left.{ }^{3} J_{\mathrm{HH}}=3 \mathrm{~Hz},{ }^{3} J_{\mathrm{HH}}=3 \mathrm{~Hz}, \mathrm{C}_{4} \underline{H}_{3} \mathrm{O}\right), 6.12\left(\mathrm{~d}, 2 \mathrm{H},{ }^{3} J_{\mathrm{HH}}=3 \mathrm{~Hz}, \mathrm{C}_{4} \underline{H}_{3} \mathrm{O}\right), 5.13(\mathrm{~d}, 2 \mathrm{H}$, $\left.{ }^{2} J_{\mathrm{HH}}=15 \mathrm{~Hz}, \underline{\mathrm{H}}_{2}\right), 4.84\left(\mathrm{~d}, 2 \mathrm{H},{ }^{2} J_{\mathrm{HH}}=15 \mathrm{~Hz}, \underline{\mathrm{C}}_{2}\right), 4.50\left(\mathrm{~d}, 2 \mathrm{H},{ }^{2} J_{\mathrm{HH}}=15 \mathrm{~Hz}\right.$, $\left.\mathrm{C} \underline{H}_{2}\right), 3.61\left(\mathrm{~d}, 2 \mathrm{H},{ }^{2} J_{\mathrm{HH}}=15 \mathrm{~Hz}, \underline{\mathrm{C}}_{2}\right), 3.54\left(\mathrm{qd}, 2 \mathrm{H},{ }^{3} \mathrm{~J}_{\mathrm{HH}}=7 \mathrm{~Hz}, \underline{\mathrm{C}}_{2} \mathrm{CH}_{3}\right), 3.16(\mathrm{qd}$, $\left.2 \mathrm{H},{ }^{3} \mathrm{~J}_{\mathrm{HH}}=7 \mathrm{~Hz}, \underline{\mathrm{C}}_{2} \mathrm{CH}_{3}\right), 1.23\left(\mathrm{t}, 6 \mathrm{H},{ }^{3} \mathrm{~J}_{\mathrm{HH}}=7 \mathrm{~Hz}, \mathrm{CH}_{2} \underline{\mathrm{C}}_{3}\right) .{ }^{13} \mathrm{C}\left\{{ }^{1} \mathrm{H}\right\} \mathrm{NMR}$ $\left(\mathrm{CDCl}_{3}, 100 \mathrm{MHz}, 25^{\circ} \mathrm{C}\right): \delta 171.0(\mathrm{Ni}-\mathrm{N} \underline{C} \mathrm{~N}), 168.3(\underline{C}=\mathrm{O}), 156.6\left(\underline{C}_{4} \mathrm{H}_{3} \mathrm{O}\right), 142.4$ $(\mathrm{N}-\underline{C}(3)-\mathrm{N}), 141.1 \quad\left(\underline{C}_{4} \mathrm{H}_{3} \mathrm{O}\right), 110.9\left(\underline{C}_{4} \mathrm{H}_{3} \mathrm{O}\right), 106.3\left(\underline{C}_{4} \mathrm{H}_{3} \mathrm{O}\right), 54.3\left(\underline{C}_{2}\right), 46.9$ $\left(\underline{\mathrm{C}} \mathrm{H}_{2} \mathrm{CH}_{3}\right), 41.8\left(\underline{\mathrm{C}} \mathrm{H}_{2}\right), 15.1\left(\mathrm{CH}_{2} \underline{C} \mathrm{H}_{3}\right)$. IR data $(\mathrm{KBr}$ pellet $) \mathrm{cm}^{-1}: 1590(\mathrm{~s})\left(v_{\mathrm{c}=\mathrm{o}}\right)$. HRMS (ES): $m / z$ 525.1506 $[\mathrm{M}+\mathrm{H}]^{+}$, calcd. 525.1503. Anal. Calcd. for $\mathrm{C}_{22} \mathrm{H}_{26} \mathrm{NiN}_{8} \mathrm{O}_{4} \bullet \mathrm{H}_{2} \mathrm{O}: \mathrm{C}, 48.64 ; \mathrm{H}, 5.20 ; \mathrm{N}, 20.63$. Found: $\mathrm{C}, 48.42 ; \mathrm{H}, 5.45 ; \mathrm{N}, 20.30$ $\%$.

\section{Synthesis of 1-(i-propyl)-4-N-(furan-2-ylmethyl)acetamido-1,2,4-triazolium chloride (2a)}

A mixture of $i$-propyl triazole (1.96 g, $17.7 \mathrm{mmol})$ and 2-chloro-N-(furan-2ylmethyl)acetamide $(3.06 \mathrm{~g}, 17.6 \mathrm{mmol})$ was refluxed in toluene $(\mathrm{ca} .30 \mathrm{~mL})$ for 14 hours during which a black viscous precipitate was formed. After decanting off the solvent, the precipitate was washed with hot petroleum ether $(3 \times 10 \mathrm{~mL})$ and then dissolved in minimum amount of dichloromethane $(c a .3 \mathrm{~mL})$. The product was precipitated out by addition of diethyl ether $(c a .15 \mathrm{~mL})$. The product was isolated by decantation and was vacuum dried to give the product (2a) as a hygroscopic black 
solid (2.65 g, $53 \%) .{ }^{1} \mathrm{H}$ NMR (DMSO- $\left.d_{6}, 400 \mathrm{MHz}, 25{ }^{\circ} \mathrm{C}\right): \delta 10.2(\mathrm{~s}, 1 \mathrm{H}, \mathrm{N}-\mathrm{C}(5) \underline{H}-$ N), 9.14 (s, $1 \mathrm{H}, \mathrm{N}-\mathrm{C}(3) \underline{H}-\mathrm{N}), 9.09$ (br, $1 \mathrm{H}, \mathrm{N} \underline{H C O}), 7.58$ (br, $\left.1 \mathrm{H}, \mathrm{C}_{4} \underline{H}_{3} \mathrm{O}\right), 6.40$ (dd, $\left.1 \mathrm{H},{ }^{3} J_{\mathrm{HH}}=3 \mathrm{~Hz},{ }^{3} J_{\mathrm{HH}}=3 \mathrm{~Hz}, \mathrm{C}_{4} \underline{H}_{3} \mathrm{O}\right), 6.31\left(\mathrm{~d}, 1 \mathrm{H},{ }^{3} J_{\mathrm{HH}}=3 \mathrm{~Hz}, \mathrm{C}_{4} \underline{H}_{3} \mathrm{O}\right), 5.11(\mathrm{~s}, 2 \mathrm{H}$, $\left.\mathrm{C}_{2}\right), 4.86$ (sept, $\left.1 \mathrm{H},{ }^{3} J_{\mathrm{HH}}=7 \mathrm{~Hz}, \underline{\mathrm{C}} \underline{H}\left(\mathrm{CH}_{3}\right)_{2}\right), 4.34\left(\mathrm{~d}, 2 \mathrm{H},{ }^{3} J_{\mathrm{HH}}=5 \mathrm{~Hz}, \underline{\mathrm{C}}_{2} \mathrm{NH}\right)$, $1.50\left(\mathrm{~d}, 6 \mathrm{H},{ }^{3} J_{\mathrm{HH}}=7 \mathrm{~Hz}, \mathrm{CH}\left(\mathrm{C}_{3}\right)_{2}\right) .{ }^{13} \mathrm{C}\left\{{ }^{1} \mathrm{H}\right\} \mathrm{NMR}\left(\mathrm{DMSO}-d_{6}, 100 \mathrm{MHz}, 25^{\circ} \mathrm{C}\right): \delta$ $164.4(\underline{C}=\mathrm{O}), 151.4\left(\underline{C}_{4} \mathrm{H}_{3} \mathrm{O}\right), 145.8(\mathrm{~N}-\underline{C}(5)-\mathrm{N}), 142.7(\mathrm{~N}-\underline{C}(3)-\mathrm{N}), 142.4\left(\underline{C}_{4} \mathrm{H}_{3} \mathrm{O}\right)$, $110.8\left(\underline{C}_{4} \mathrm{H}_{3} \mathrm{O}\right), 107.9\left(\underline{C}_{4} \mathrm{H}_{3} \mathrm{O}\right), 55.5\left(\underline{\mathrm{CH}}\left(\mathrm{CH}_{3}\right)_{2}\right), 49.0\left(\underline{\mathrm{C}} \mathrm{H}_{2}\right), 36.1\left(\underline{\mathrm{C}} \mathrm{H}_{2}\right), 21.4$

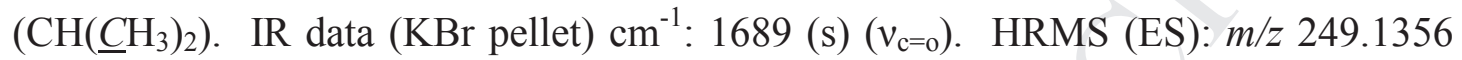
$[\mathrm{NHC}+\mathrm{H}]^{+}$, calcd. 249.1352. Anal. Calcd. for $\mathrm{C}_{12} \mathrm{H}_{17} \mathrm{~N}_{4} \mathrm{O}_{2} \mathrm{Cl} \bullet 0.5 \mathrm{H}_{2} \mathrm{O}: \mathrm{C}, 49.07 ; \mathrm{H}$, 6.18; N, 19.07. Found: C, 48.63; H, 6.74; N, $17.97 \%$.

\section{Synthesis of [1-(i-propyl)-4-N-(furan-2-ylmethyl)acetamido-1,2,4-triazol-5-} ylidene $]_{2} \mathrm{Ni}(2 \mathrm{~b})$

A mixture of 1-(i-propyl)-4-N-(furan-2-ylmethyl)acetamido-1,2,4-triazolium chloride (2a) $(2.33 \mathrm{~g}, 8.20 \mathrm{mmol}), \mathrm{K}_{2} \mathrm{CO}_{3}(5.65 \mathrm{~g}, 40.9 \mathrm{mmol})$ and $\mathrm{NiCl}_{2} \bullet 6 \mathrm{H}_{2} \mathrm{O}(0.974 \mathrm{~g}, 4.09$ mmol) was refluxed in acetonitrile $(c a .70 \mathrm{~mL})$ for 24 hours. The reaction mixture was filtered and filtrate was reduced in vacuum up to $15 \mathrm{~mL}$. The filtrate was kept at room temperature for overnight, during which brown crystals were formed. Finally the crystals were separated by filtration and vacuum dried to give the product $(\mathbf{2 b})$ as a yellow crystalline solid (0.684 g, $30 \%) .{ }^{1} \mathrm{H}$ NMR $\left(\mathrm{CDCl}_{3}, 400 \mathrm{MHz}, 25^{\circ} \mathrm{C}\right): \delta 8.07$ (s, $2 \mathrm{H}, \mathrm{N}-\mathrm{C}(3) \underline{H}-\mathrm{N}), 7.29\left(\mathrm{br}, 2 \mathrm{H}, \mathrm{C}_{4} \underline{H}_{3} \mathrm{O}\right), 6.31\left(\mathrm{dd}, 2 \mathrm{H},{ }^{3} J_{\mathrm{HH}}=3 \mathrm{~Hz},{ }^{3} J_{\mathrm{HH}}=3 \mathrm{~Hz}\right.$, $\left.\mathrm{C}_{4} \underline{H}_{3} \mathrm{O}\right), 6.13\left(\mathrm{~d}, 2 \mathrm{H},{ }^{3} J_{\mathrm{HH}}=3 \mathrm{~Hz}, \mathrm{C}_{4} \underline{H}_{3} \mathrm{O}\right), 5.24\left(\mathrm{~d}, 2 \mathrm{H},{ }^{2} J_{\mathrm{HH}}=15 \mathrm{~Hz}, \underline{\mathrm{C}}_{2}\right), 4.83(\mathrm{~d}$, $\left.2 \mathrm{H},{ }^{2} J_{\mathrm{HH}}=15 \mathrm{~Hz}, \underline{\mathrm{C}}_{2}\right), 4.57\left(\mathrm{~d}, 2 \mathrm{H},{ }^{2} J_{\mathrm{HH}}=15 \mathrm{~Hz}, \underline{\mathrm{C}}_{2}\right), 3.59\left(\mathrm{~d}, 2 \mathrm{H},{ }^{2} J_{\mathrm{HH}}=15 \mathrm{~Hz}\right.$,

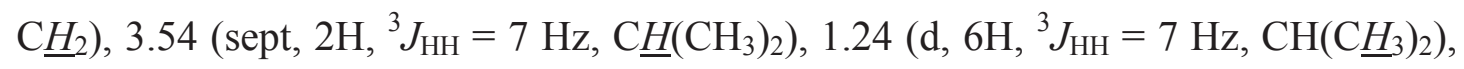
$1.14\left(\mathrm{~d}, 6 \mathrm{H},{ }^{3} \mathrm{~J}_{\mathrm{HH}}=7 \mathrm{~Hz}, \mathrm{CH}\left(\mathrm{C}_{3}\right)_{2}\right) .{ }^{13} \mathrm{C}\left\{{ }^{1} \mathrm{H}\right\} \mathrm{NMR}\left(\mathrm{CDCl}_{3}, 100 \mathrm{MHz}, 25^{\circ} \mathrm{C}\right): \delta$ 
$171.2(\mathrm{Ni}-\mathrm{N} \underline{C} \mathrm{~N}), 168.5(\underline{C}=\mathrm{O}), 156.2\left(\underline{C}_{4} \mathrm{H}_{3} \mathrm{O}\right), 142.3(\mathrm{~N}-\underline{C}(3)-\mathrm{N}), 141.1\left(\underline{C}_{4} \mathrm{H}_{3} \mathrm{O}\right)$, $110.7\left(\underline{C}_{4} \mathrm{H}_{3} \mathrm{O}\right), 106.7\left(\underline{C}_{4} \mathrm{H}_{3} \mathrm{O}\right), 54.3\left(\underline{\mathrm{CH}}\left(\mathrm{CH}_{3}\right)_{2}\right), 54.2\left(\underline{\mathrm{C}} \mathrm{H}_{2}\right), 41.8\left(\underline{\mathrm{C}} \mathrm{H}_{2}\right), 24.3$ $\left(\mathrm{CH}\left(\underline{\mathrm{CH}}_{3}\right)_{2}\right), 20.3\left(\mathrm{CH}\left(\underline{\mathrm{CH}}_{3}\right)_{2}\right)$. IR data $(\mathrm{KBr}$ pellet$) \mathrm{cm}^{-1}: 1596(\mathrm{~s})\left(\mathrm{v}_{\mathrm{c}=\mathrm{o}}\right)$. HRMS (ES): $m / z$ 553.1817 $[\mathrm{M}+\mathrm{H}]^{+}$, calcd. 553.1816. Anal. Calcd. for $\mathrm{C}_{24} \mathrm{H}_{30} \mathrm{NiN}_{8} \mathrm{O}_{4}: \mathrm{C}$, 52.10; H, 5.47; N, 20.25. Found: C, 51.44; H, 6.04; N, $20.28 \%$.

\section{Synthesis of 1-(benzyl)-4-N-(furan-2-ylmethyl)acetamido-1,2,4-triazolium chloride (3a)}

A mixture of benzyl triazole (2.05 g, $12.9 \mathrm{mmol})$ and 2-chloro-N-(furan-2ylmethyl)acetamide $(2.21 \mathrm{~g}, 12.8 \mathrm{mmol})$ was refluxed in toluene $(\mathrm{ca} .40 \mathrm{~mL})$ for 32 hours during which a black precipitate was formed. After decanting off the solvent, the precipitate was washed with hot petroleum ether $(c a .3 \times 10 \mathrm{~mL})$ and then dissolved in minimum amount of dichloromethane $(c a .4 \mathrm{~mL})$. The product was precipitated out by addition of diethyl ether $(2 \times 15 \mathrm{~mL})$. The product was finally isolated by decantation and was vacuum dried to give the product (3a) as a black solid $(2.20 \mathrm{~g}, 52 \%) .{ }^{1} \mathrm{H}$ NMR (DMSO- $\left.d_{6}, 400 \mathrm{MHz}, 25{ }^{\circ} \mathrm{C}\right): \delta 10.3(\mathrm{~s}, 1 \mathrm{H}, \mathrm{N}-\mathrm{C}(5) \underline{H}-\mathrm{N})$, 9.20 (br, $1 \mathrm{H}, \mathrm{N}-\mathrm{C}(3) \underline{H}-\mathrm{N}), 9.16$ (s, $1 \mathrm{H}, \mathrm{N} \underline{H C O}), 7.60$ (br, 1H, $\left.\mathrm{C}_{4} \underline{H}_{3} \mathrm{O}\right), 7.43$ (br, 5H, $\left.\mathrm{C}_{6} \underline{H}_{5}\right), 6.41\left(\mathrm{dd}, 1 \mathrm{H},{ }^{3} J_{\mathrm{HH}}=3 \mathrm{~Hz},{ }^{3} J_{\mathrm{HH}}=3 \mathrm{~Hz}, \mathrm{C}_{4} \underline{H}_{3} \mathrm{O}\right), 6.32\left(\mathrm{~d}, 1 \mathrm{H},{ }^{3} J_{\mathrm{HH}}=3 \mathrm{~Hz}\right.$, $\left.\mathrm{C}_{4} \underline{H}_{3} \mathrm{O}\right), 5.70\left(\mathrm{~s}, 2 \mathrm{H}, \underline{\mathrm{C}}_{2}\right), 5.17\left(\mathrm{~s}, 2 \mathrm{H}, \underline{\mathrm{C}}_{2}\right), 4.34\left(\mathrm{~d}, 2 \mathrm{H},{ }^{3} J_{\mathrm{HH}}=6 \mathrm{~Hz}, \underline{\mathrm{C}}_{2} \mathrm{NH}\right)$. ${ }^{13} \mathrm{C}\left\{{ }^{1} \mathrm{H}\right\}$ NMR (DMSO- $\left.d_{6}, 100 \mathrm{MHz}, 25{ }^{\circ} \mathrm{C}\right): \delta 164.3(\underline{C}=\mathrm{O}), 151.3\left(\underline{C}_{4} \mathrm{H}_{3} \mathrm{O}\right), 146.1$ $(\mathrm{N}-\underline{C}(5)-\mathrm{N}), 143.8\left(\underline{C}_{6} \mathrm{H}_{5}\right), 142.4(\mathrm{~N}-\underline{C}(3)-\mathrm{N}), 142.4\left(\underline{C}_{4} \mathrm{H}_{3} \mathrm{O}\right), 133.4\left(\underline{C}_{6} \mathrm{H}_{5}\right), 128.9$ $\left(\underline{C}_{6} \mathrm{H}_{5}\right) 128.8\left(\underline{C}_{6} \mathrm{H}_{5}\right), 110.5\left(\underline{C}_{4} \mathrm{H}_{3} \mathrm{O}\right), 107.5\left(\underline{C}_{4} \mathrm{H}_{3} \mathrm{O}\right), 54.7\left(\underline{C H}_{2}\right), 49.1\left(\underline{C H}_{2}\right), 35.9$ $\left(\underline{C}_{2}\right) . \quad$ IR data $\left(\mathrm{KBr}\right.$ pellet) $\mathrm{cm}^{-1}: 1669(\mathrm{~s})\left(v_{\mathrm{c}=\mathrm{o}}\right) . \quad \mathrm{HRMS}(\mathrm{ES}): \mathrm{m} / z 297.1350$ $[\mathrm{NHC}+\mathrm{H}]^{+}$, calcd. 297.1352. Anal. Calcd. for $\mathrm{C}_{16} \mathrm{H}_{17} \mathrm{~N}_{4} \mathrm{O}_{2} \mathrm{Cl} \bullet 0.5 \mathrm{H}_{2} \mathrm{O}: \mathrm{C}, 56.22 ; \mathrm{H}$, 5.31; N, 16.39. Found: C, 55.93; H, 5.83; N, $15.37 \%$. 


\section{Synthesis of [1-(benzyl)-4-N-(furan-2-ylmethyl)acetamido-1,2,4-triazol-5-} ylidene $]_{2} \mathrm{Ni}(3 \mathrm{~b})$

A mixture of 1-benzyl-4-N-(furan-2-ylmethyl)acetamido-1,2,4-triazolium chloride (3a) (1.20g, $3.60 \mathrm{mmol}), \mathrm{K}_{2} \mathrm{CO}_{3}(2.48 \mathrm{~g}, 18.0 \mathrm{mmol})$ and $\mathrm{NiCl}_{2} \cdot 6 \mathrm{H}_{2} \mathrm{O}(0.428 \mathrm{~g}, 1.80$ mmol) was refluxed in acetonitrile $(c a .50 \mathrm{~mL})$ for 24 hours. The reaction mixture was filtered and the filtrate reduced in vacuum up to $10 \mathrm{~mL}$. The filtrate was kept at $0^{\circ} \mathrm{C}$ for 3 hours, during which yellow crystals were formed. Finally the crystals were quickly separated by filtration and vacuum dried to give the product (3b) as a yellow solid $(0.163 \mathrm{~g}, 14 \%) .{ }^{1} \mathrm{H}$ NMR $\left(\mathrm{CDCl}_{3}, 400 \mathrm{MHz}, 25^{\circ} \mathrm{C}\right): \delta 8.12(\mathrm{~s}, 2 \mathrm{H}, \mathrm{N}-\mathrm{C}(3) \underline{H}-$ N), $7.31\left(\mathrm{~m}, 6 \mathrm{H}, \mathrm{C}_{6} \underline{H}_{5}\right), 7.19\left(\mathrm{br}, 2 \mathrm{H}, \mathrm{C}_{4} \underline{H}_{3} \mathrm{O}\right), 6.91\left(\mathrm{~d}, 4 \mathrm{H},{ }^{3} \mathrm{~J}_{\mathrm{HH}}=7 \mathrm{~Hz}, \mathrm{C}_{6} \underline{H}_{5}\right), 6.29$ (br, $\left.2 \mathrm{H}, \mathrm{C}_{4} \underline{H}_{3} \mathrm{O}\right), 6.05\left(\mathrm{~d}, 2 \mathrm{H},{ }^{3} J_{\mathrm{HH}}=2 \mathrm{~Hz}, \mathrm{C}_{4} \underline{H}_{3} \mathrm{O}\right), 4.97\left(\mathrm{~d}, 2 \mathrm{H},{ }^{2} J_{\mathrm{HH}}=15 \mathrm{~Hz}, \underline{\mathrm{C}}_{2}\right)$, $4.79\left(\mathrm{~d}, 2 \mathrm{H},{ }^{2} J_{\mathrm{HH}}=15 \mathrm{~Hz}, \underline{\mathrm{C}}_{2}\right), 4.50\left(\mathrm{~d}, 2 \mathrm{H},{ }^{2} J_{\mathrm{HH}}=15 \mathrm{~Hz}, \underline{\mathrm{C}}_{2}\right), 4.42\left(\mathrm{~d}, 2 \mathrm{H},{ }^{2} J_{\mathrm{HH}}=\right.$ $\left.15 \mathrm{~Hz}, \underline{\mathrm{C}}_{2}\right), 4.39\left(\mathrm{~d}, 2 \mathrm{H},{ }^{2} J_{\mathrm{HH}}=15 \mathrm{~Hz}, \underline{\mathrm{C}}_{2}\right), 3.62\left(\mathrm{~d}, 2 \mathrm{H},{ }^{2} J_{\mathrm{HH}}=15 \mathrm{~Hz}, \underline{\mathrm{C}}_{2}\right)$. ${ }^{13} \mathrm{C}\left\{{ }^{1} \mathrm{H}\right\}$ NMR $\left(\mathrm{CDCl}_{3}, 100 \mathrm{MHz}, 25^{\circ} \mathrm{C}\right): \delta 171.6(\mathrm{Ni}-\mathrm{N} \underline{C} \mathrm{~N}), 168.4(\underline{C}=\mathrm{O}), 156.0$ $\left(\underline{C}_{4} \mathrm{H}_{3} \mathrm{O}\right), 142.7(\mathrm{~N}-\underline{C}(3)-\mathrm{N}), 141.2\left(\underline{C}_{4} \mathrm{H}_{3} \mathrm{O}\right), 133.6\left(\underline{C}_{6} \mathrm{H}_{5}\right), 129.3\left(\underline{C}_{6} \mathrm{H}_{5}\right), 129.0$ $\left(\underline{C}_{6} \mathrm{H}_{5}\right), 127.6\left(\underline{C}_{6} \mathrm{H}_{5}\right), 110.7\left(\underline{C}_{4} \mathrm{H}_{3} \mathrm{O}\right), 106.3\left(\underline{C}_{4} \mathrm{H}_{3} \mathrm{O}\right), 55.4\left(\underline{C H}_{2}\right), 54.0\left(\underline{C H}_{2}\right), 42.3$ $\left(\underline{C}_{2}\right) . \quad$ IR data $(\mathrm{KBr}$ pellet $) \mathrm{cm}^{-1}: 1595(\mathrm{~s})\left(v_{\mathrm{c}=\mathrm{o}}\right)$. HRMS (ES): $\mathrm{m} / z 649.1813$ $[\mathrm{M}+\mathrm{H}]^{+}$, calcd. 649.1816. Anal. Calcd. for $\mathrm{C}_{32} \mathrm{H}_{30} \mathrm{NiN}_{8} \mathrm{O}_{4} \bullet \mathrm{H}_{2} \mathrm{O}: \mathrm{C}, 57.59 ; \mathrm{H}, 4.83$; N, 16.79. Found: C, 57.71; H, 5.05; N, $16.74 \%$.

\section{Computational Methods}

Density functional theory (DFT) calculations were performed on the metal complexes (1-3)b using GAUSSIAN 09 [22] suite of quantum chemical programs. The Becke three parameter exchange functional in conjunction with Lee-Yang-Parr correlation 
functional (B3LYP) has been employed in the study [23]. The polarized basis set 6$31 \mathrm{G}(\mathrm{d})$ [24] was used to describe oxygen, carbon, nitrogen and hydrogen atoms. The Stuttgart-Dresden effective core potential (ECP) along with valence basis sets (LANL2DZ) was used for the nickel [25]. Natural bond orbital (NBO) analysis [26] was performed using NBO 3.1 program implemented in the GAUSSIAN 09 package. Frequency calculations were performed for all the optimized structures to characterize the stationary points as minima.

The metal-ligand donor-acceptor interaction were inspected by using the Charge Decomposition Analysis (CDA) [27] which is a valuable tool for analyzing the interactions between molecular fragments on a quantitative basis with an emphasis on the electron donation [28]. The orbital interaction between donor NHC and acceptor fragment $\mathrm{Ni}^{2+}$ ion in $(\mathbf{1}-\mathbf{3}) \mathbf{b}$ can be divided into three parts;

(i) $\sigma$-donation from two mono-anionic NHC ligand fragments to a nickel(II) ion $\left\{2[\mathrm{NHC}]^{-} \rightarrow \mathrm{Ni}^{2+}\right\}$ and designated by $(d)$,

(ii) $\pi$ - back donation from a nickel(II) in to two mono-anionic NHC ligand fragments $\left\{2[\mathrm{NHC}]^{-} \leftarrow \mathrm{Ni}^{2+}\right\}$ and designated by $(b)$ and

(iii) A repulsive interaction $(r)$ between the occupied FMOs of these two fragments.

The CDA calculations were performed using the AOMix $[29,30]$ program with the B3LYP/ LANL2DZ, 6-31G(d) wave function. Molecular orbital (MO) compositions and the overlap populations were calculated using the AOMix program. Analysis of the MO compositions in terms of occupied and unoccupied fragment orbitals (OFOs 
and UFOs, respectively), construction of orbital interaction diagrams, the charge decomposition analysis (CDA) were performed using the AOMix-CDA [31].

\section{Catalysis studies}

General procedure: In a Schlenk tube, under argon atmosphere, was introduced the arylbromide (0.25 mmol, 1 equiv.), $\mathrm{Cs}_{2} \mathrm{CO}_{3}$ (261 mg, $0.80 \mathrm{mmol}, 3$ equiv.), bis(pinacolato)diboron (102 $\mathrm{mg}, 0.40 \mathrm{mmol}, 1.5$ equiv.), the nickel complex $\mathbf{1 b}$ (7 $\mathrm{mg}, 1.3 \mathrm{mmol}, 5 \mathrm{~mol} \%$ ) and $1 \mathrm{~mL}$ of dry THF. The reaction mixture was then stirred at $70{ }^{\circ} \mathrm{C}$ for $20 \mathrm{~h}$. The THF was carefully evaporated. The conversion of the arylbromide and the yield of the borylated arene were determinated by ${ }^{1} \mathrm{H}-\mathrm{NMR}$ using 1,3,5-trimethoxybenzene ( $22 \mathrm{mg}, 0.13 \mathrm{mmol}, 0.5$ equiv.) as an internal standard.

\section{Acknowledgements}

We thank Indo-French Centre for the Promotion of Advanced Research - IFCPAR (Project No: 4605-1), New Delhi, for the financial support of this research. PG is grateful to the Sophisticated Analytical Instrument Facility at IIT Bombay for the characterization data and to the National Single Crystal X-ray Diffraction Facility, IIT Bombay, and the Single Crystal X-ray Diffraction Facility at the Chemistry Department, IIT Bombay, for the crystallographic data. The computational facilities at the Computer Center, IIT Bombay, and at the Chemistry Department, IIT Bombay, are gratefully acknowledged. AK thanks CSIR, New Delhi, for research fellowship.

CD and JBS thank the French "Ministère de l'Enseignement Supérieur et de la Recherche" and the CNRS for financial support. LPB thanks IFCPAR for a $\mathrm{PhD}$ grant. 
Electronic supplementary information (ESI) available: The characterization data of the (1-3)b complexes (Supporting Information Figure S1-S33), the B3LYP coordinates of the optimized geometries for $(\mathbf{1}-\mathbf{3}) \mathbf{b}$, NBO tables and CDA table along with orbital interaction diagrams of $\mathbf{2} \mathbf{b}$ and $\mathbf{3 b}$. This material is available free of charge via the internet at http:www.Xxxxxxxxx 


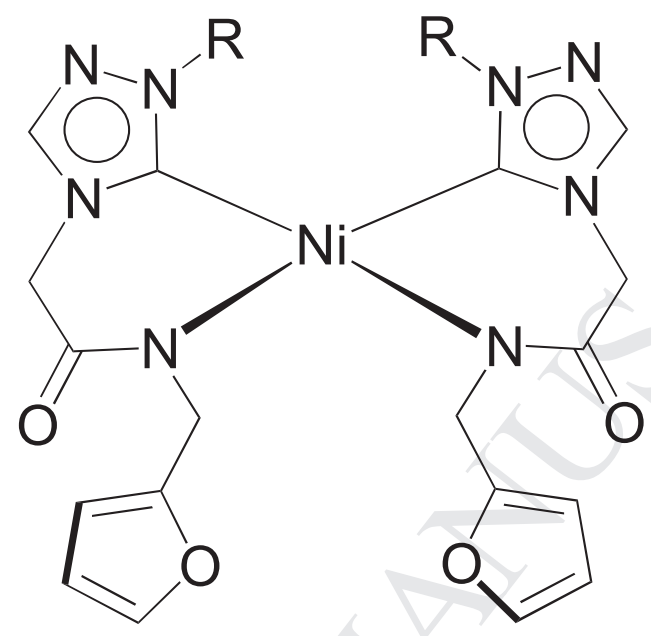

$\mathrm{R}=\mathrm{Et}(\mathbf{1} \mathbf{b}), i-\operatorname{Pr}(\mathbf{2 b}), \quad \mathrm{Bn}(\mathbf{3 b})$

Figure 1. 

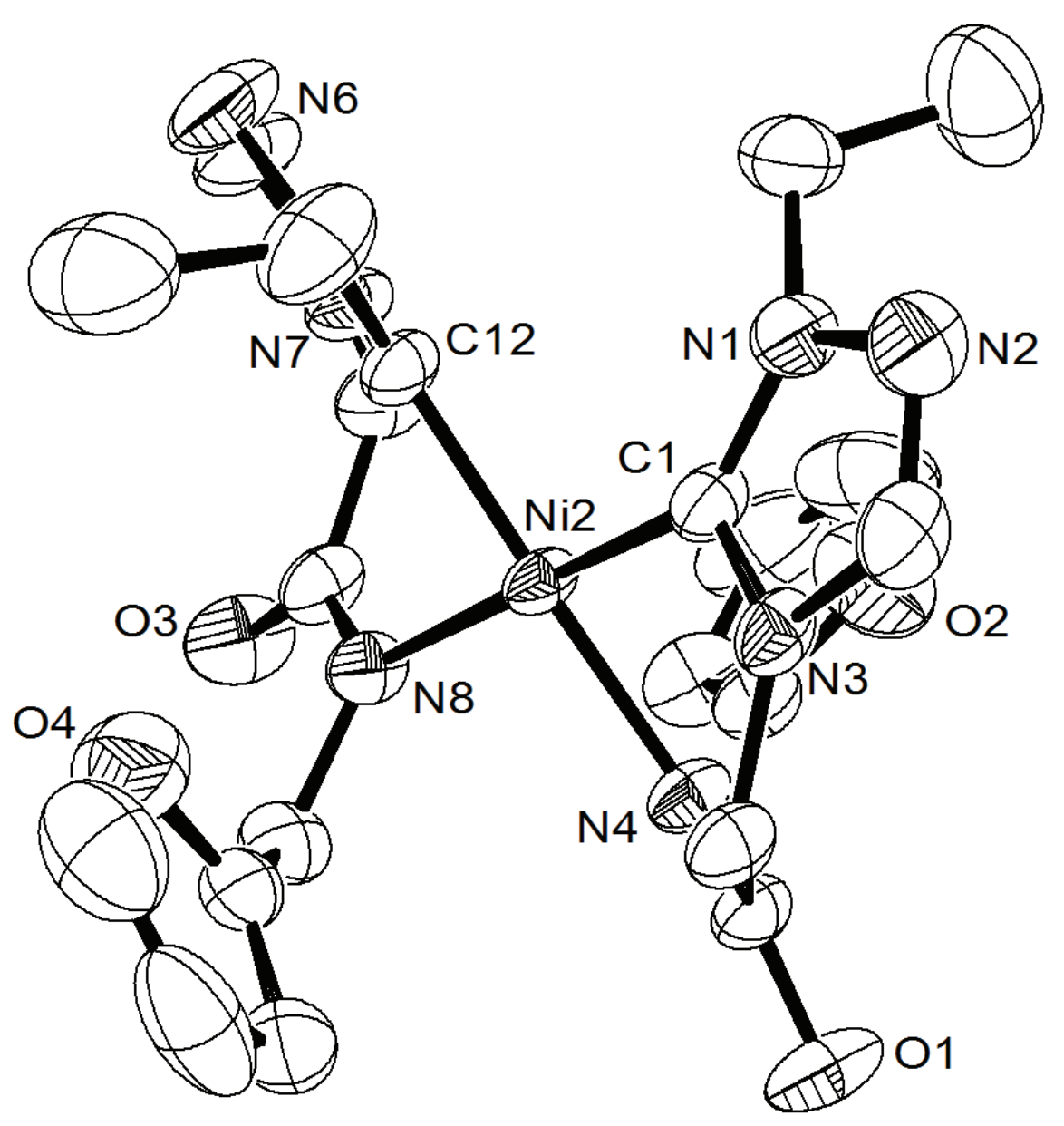

Figure 2. ORTEP view of $\mathbf{1 b}$ with thermal ellipsoids are shown at the $50 \%$ probability level. Selected bond lengths $(\AA)$ and angles $\left({ }^{\circ}\right)$ : Ni(2)-C(1) 1.860(3), $\mathrm{Ni}(2)-\mathrm{C}(34) \quad 1.859(3), \quad \mathrm{Ni}(2)-\mathrm{N}(4) \quad 1.9230(2), \quad \mathrm{Ni}(2)-\mathrm{N}(8) \quad 1.9220(2)$, $\mathrm{C}(1)-\mathrm{Ni}(2)-\mathrm{N}(4) \quad 87.54(10), \quad \mathrm{C}(12)-\mathrm{Ni}(2)-\mathrm{N}(8) \quad 85.82(10), \quad \mathrm{C}(1)-\mathrm{Ni}(2)-\mathrm{C}(12)$ 94.64(11), N(4)-Ni(2)-N(8) 92.43(9). 


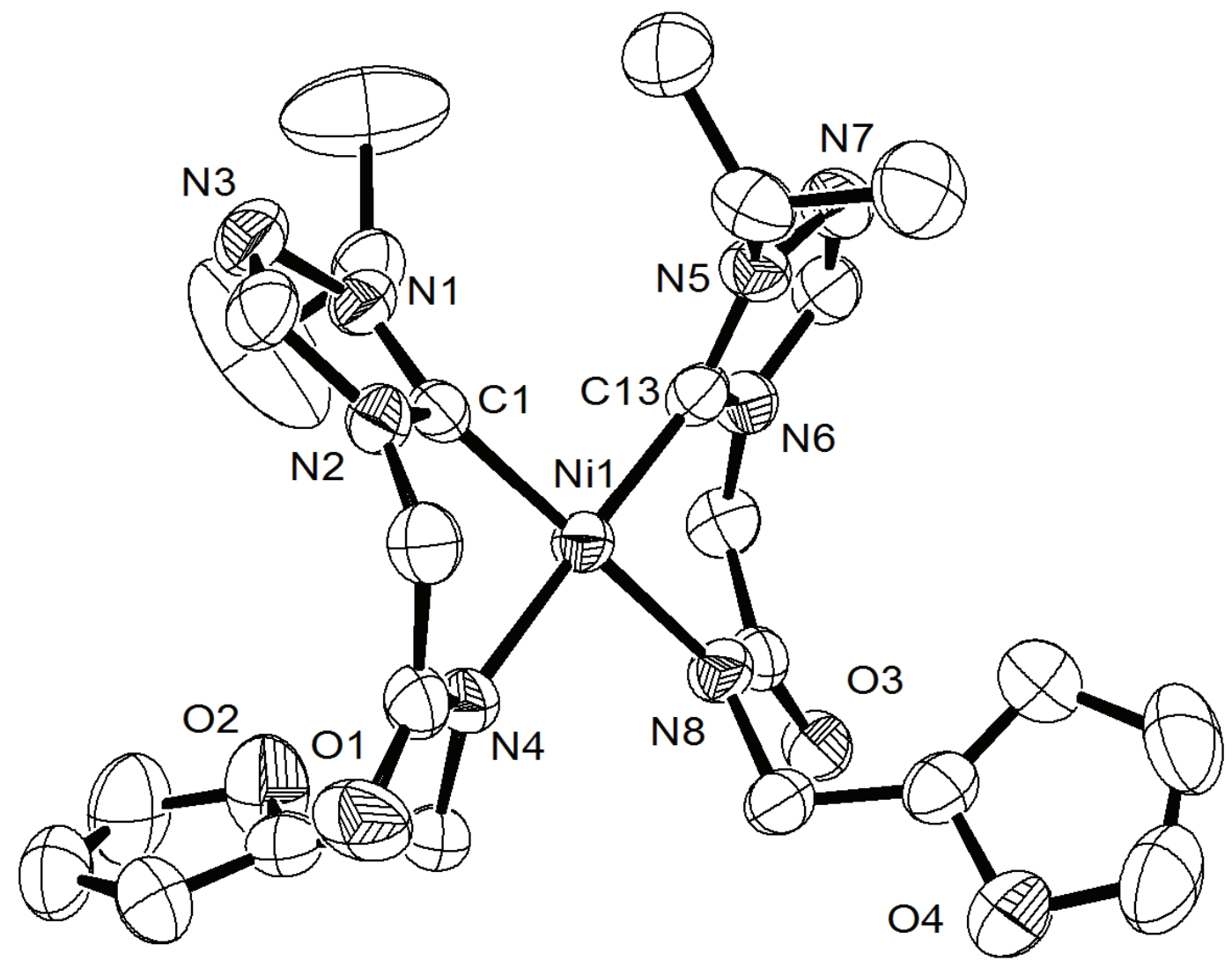

Figure 3. ORTEP view of $\mathbf{2 b}$ with thermal ellipsoids are shown at the $50 \%$ probability level. Selected bond lengths $(\AA)$ and angles $\left(^{\circ}\right)$ : Ni(1)-C(1) 1.853(2), $\mathrm{Ni}(1)-\mathrm{C}(13) \quad 1.853(2), \quad \mathrm{Ni}(1)-\mathrm{N}(4) \quad 1.9260(18), \quad \mathrm{Ni}(1)-\mathrm{N}(8) \quad 1.9211(18)$, C(1)-Ni(1)-N(4) 86.67(8), C(13)-Ni(1)-N(8) 86.21(9), C(1)-Ni(1)-C(13) 92.83(8), $\mathrm{N}(4)-\mathrm{Ni}(1)-\mathrm{N}(8)$ 94.61(7). 


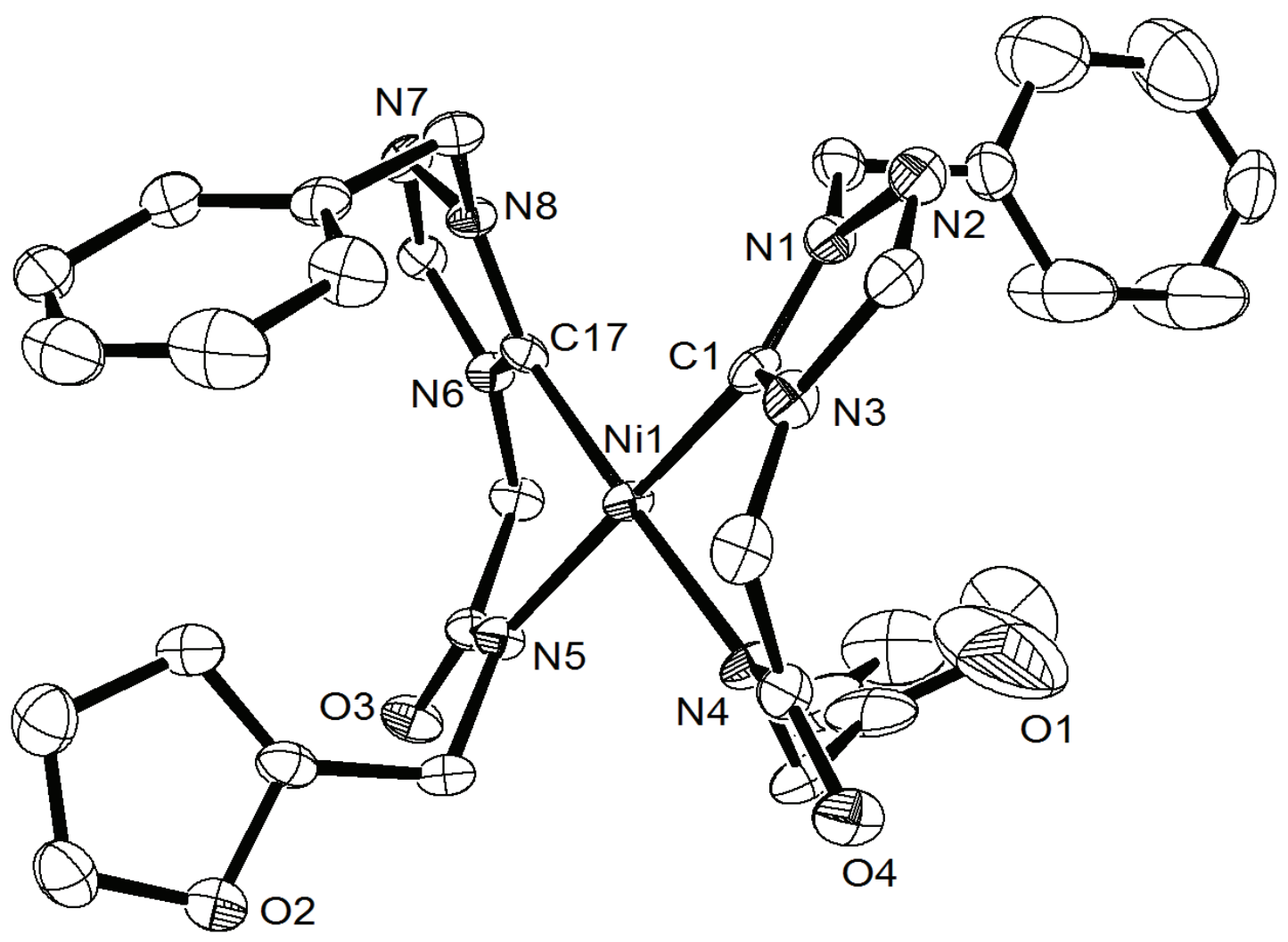

Figure 4. ORTEP view of $\mathbf{3 b}$ with thermal ellipsoids are shown at the $50 \%$ probability level. Selected bond lengths $(\AA)$ and angles $\left({ }^{\circ}\right)$ : Ni(1)-C(1) 1.871(4), $\mathrm{Ni}(1)-\mathrm{C}(17)$ 1.871(4), Ni(1)-N(4) 1.927(3), Ni(1)-N(5) 1.931(3), C(1)-Ni(1)-N(4) 86.25(14), $\quad \mathrm{C}(17)-\mathrm{Ni}(1)-\mathrm{N}(5) \quad 88.09(14), \quad \mathrm{C}(1)-\mathrm{Ni}(1)-\mathrm{C}(17) \quad 94.40(15)$, $\mathrm{N}(4)-\mathrm{Ni}(1)-\mathrm{N}(5) 91.57(13)$. 


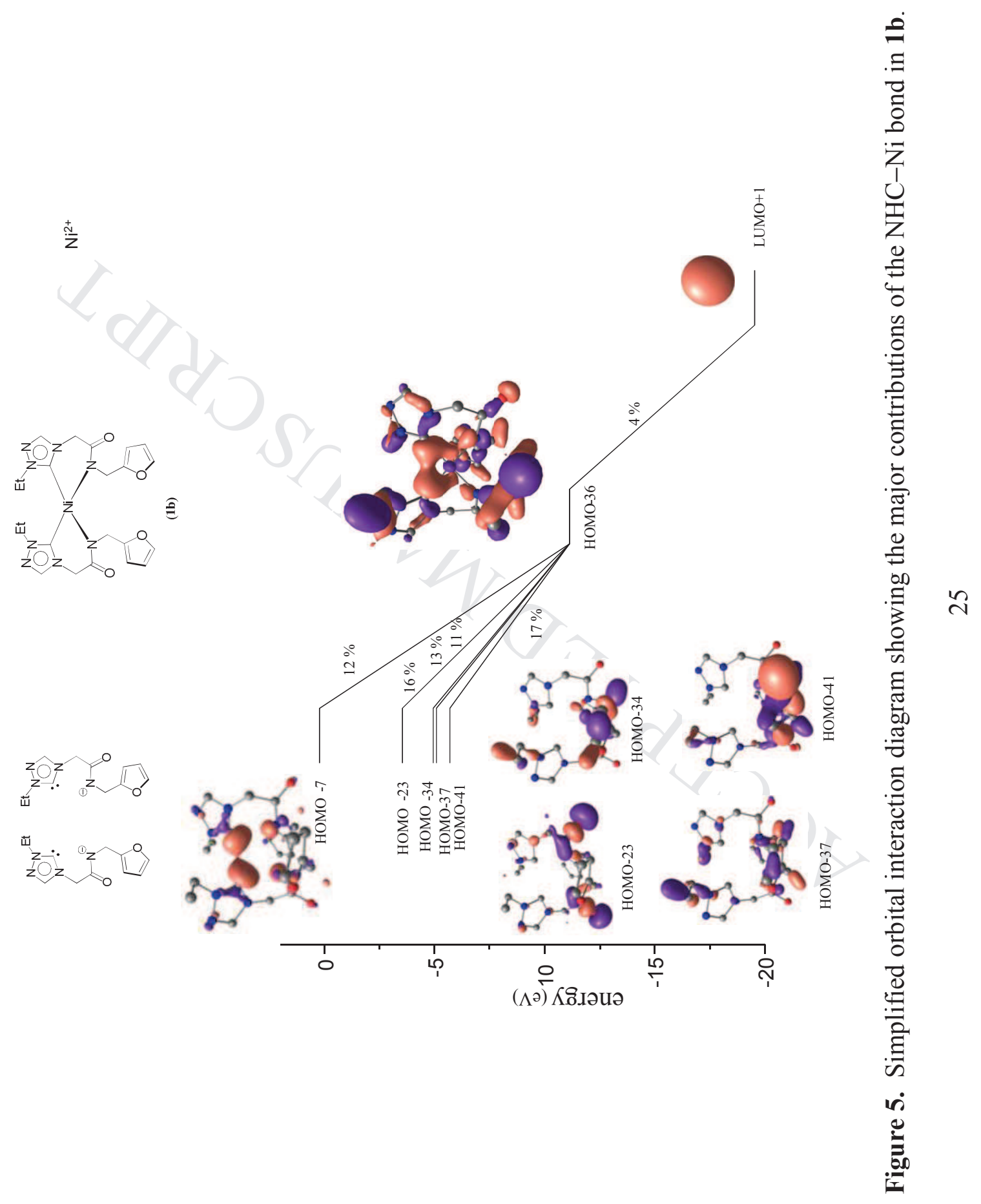



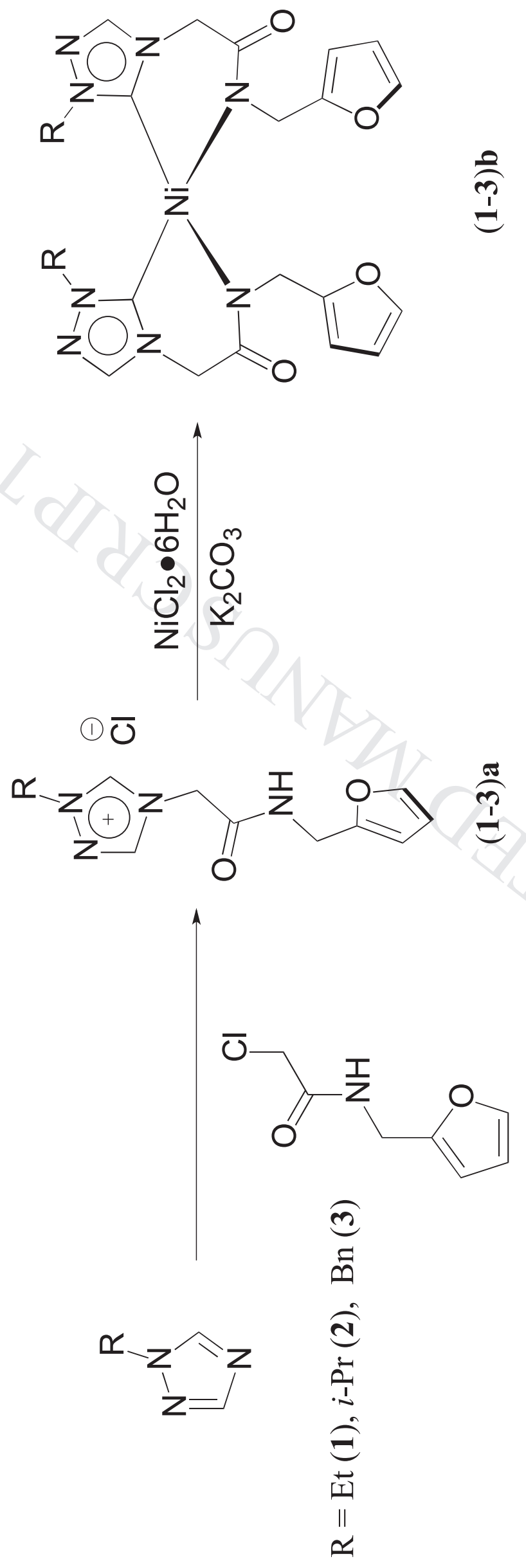

宫 
Table 1. X-ray crystallographic data for $\mathbf{1 b}, \mathbf{2 b}$ and $\mathbf{3 b}$.

\begin{tabular}{|c|c|c|c|}
\hline Compound & $1 \mathrm{~b}$ & $2 \mathbf{b}$ & $\mathbf{3 b}$ \\
\hline Lattice & Monoclinic & Orthorhombic & Monoclinic \\
\hline Formula & $\mathrm{C}_{45} \mathrm{H}_{53} \mathrm{Cl}_{3} \mathrm{~N}_{16} \mathrm{Ni}_{2} \mathrm{O}_{8.5}$ & $\mathrm{C}_{24} \mathrm{H}_{30} \mathrm{~N}_{8} \mathrm{NiO}_{4}$ & $\mathrm{C}_{32} \mathrm{H}_{30} \mathrm{~N}_{8} \mathrm{NiO}_{4}$ \\
\hline Formula weight & 1177.80 & 553.27 & 649.35 \\
\hline Space group & $P 2_{1} / \mathrm{n}$ & $P 2{ }_{1}{ }_{1} 2_{1}$ & $P 2_{1} / \mathrm{c}$ \\
\hline $\mathrm{a} / \AA$ & $18.8306(3)$ & $10.5000(2)$ & $18.590(5)$ \\
\hline $\mathrm{b} / \AA$ & $9.25340(10)$ & $13.9266(4)$ & $9.104(2)$ \\
\hline $\mathrm{c} / \AA$ & $30.7706(4)$ & $17.9794(4)$ & $18.316(5)$ \\
\hline$\alpha /^{\circ}$ & 90 & 90 & 90 \\
\hline$\beta /{ }^{\circ}$ & $94.472(2)$ & 90 & $109.49(4)$ \\
\hline$\gamma /{ }^{\circ}$ & 90 & 90 & 90 \\
\hline $\mathrm{V} / \AA^{3}$ & $5345.36(12)$ & $2629.11(11)$ & 2922.2(13) \\
\hline $\mathrm{Z}$ & 4 & 4 & 4 \\
\hline Temperature (K) & 293(2) & $293(2)$ & $100(2)$ \\
\hline Radiation $(\lambda, \AA)$ & 0.71073 & 0.71073 & 0.71073 \\
\hline$\rho$ (calcd. $), \mathrm{g} \mathrm{cm}^{-3}$ & 1.464 & 1.398 & 1.476 \\
\hline$\theta$ max, deg. & 25.00 & 25.00 & 25.00 \\
\hline No. of data & 9372 & 4620 & 7789 \\
\hline No. of parameters & 677 & 338 & 406 \\
\hline $\mathrm{R}_{1}[\mathrm{I}>2 \operatorname{sigma}(\mathrm{I})]$ & 0.0419 & 0.0277 & 0.0816 \\
\hline$w \mathrm{R}_{2}[\mathrm{I}>2 \operatorname{sigma}(\mathrm{I})]$ & 0.1149 & 0.0689 & 0.204 \\
\hline GOF & 1.038 & 1.034 & 1.109 \\
\hline
\end{tabular}


Table 2. Selected results for catalytic borylation reactions of arylbromide by bis(pinacolato)diboron catalyzed by (1-3)b.

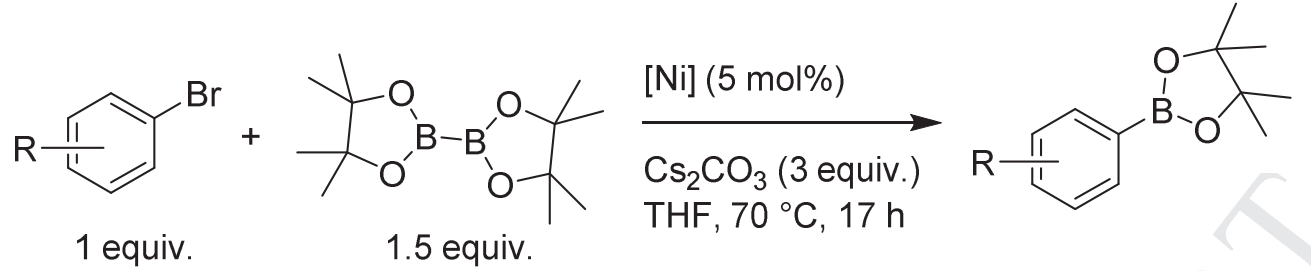

\begin{tabular}{|c|c|c|c|c|}
\hline Entry & {$[\mathrm{Ni}](5 \mathrm{~mol} \%)$} & $\mathrm{Ar}-\mathrm{Br}$ & Conversion $(\%)^{(b)}$ & Yield $(\%)^{(\mathrm{c})}$ \\
\hline 1 & $1 b$ & & 90 & 13 \\
\hline 2 & $2 b$ & & 75 & 10 \\
\hline 3 & $3 b$ & & 61 & 8 \\
\hline 4 & $1 b$ & & 88 & 12 \\
\hline 5 & $1 b$ & & 91 & 14 \\
\hline 6 & $1 b$ & & 73 & 20 \\
\hline 7 & $1 b$ & & 71 & 18 \\
\hline 8 & $1 \mathrm{~b}$ & & 83 & 10 \\
\hline 9 & $1 b$ & & 70 & 21 \\
\hline
\end{tabular}

(a) $\mathrm{Ar}-\mathrm{Br}\left(50 \mathrm{mg}, 0.25 \mathrm{mmol}, 1\right.$ equiv.), $\mathrm{Cs}_{2} \mathrm{CO}_{3}$ (261 mg, $0.80 \mathrm{mmol}, 3$ equiv.), bis(pinacolato)diboron (102 mg, $0.40 \mathrm{mmol}, 1.5$ equiv.), THF ( $1 \mathrm{~mL})$, Ni complex (7 $\mathrm{mg}, 1.3 \mathrm{mmol}, 5 \mathrm{~mol} \%$ ), $70{ }^{\circ} \mathrm{C}$ for $20 \mathrm{~h}$. (b) Conversion of the arylbromide determinated by ${ }^{1} \mathrm{H}$-NMR using 1,3,5-trimethoxybenzene ( $22 \mathrm{mg}, 0.13 \mathrm{mmol}, 0.5$ equiv.) as an internal standard. (c) NMR yield determined with1,3,5trimethoxybenzene as an internal standard. 


\section{Graphics for Table of Contents}

Nickel Complexes of 1,2,4-Triazole Derived Amido-functionalized N-heterocyclic Carbene Ligands: Synthesis, Theoretical Studies and Catalytic Application

Anuj Kumar, Linus Paulin Bheeter, Manoj Kumar Gangwar, Jean-Baptiste Sortais, Christophe Darcel* and Prasenjit Ghosh*

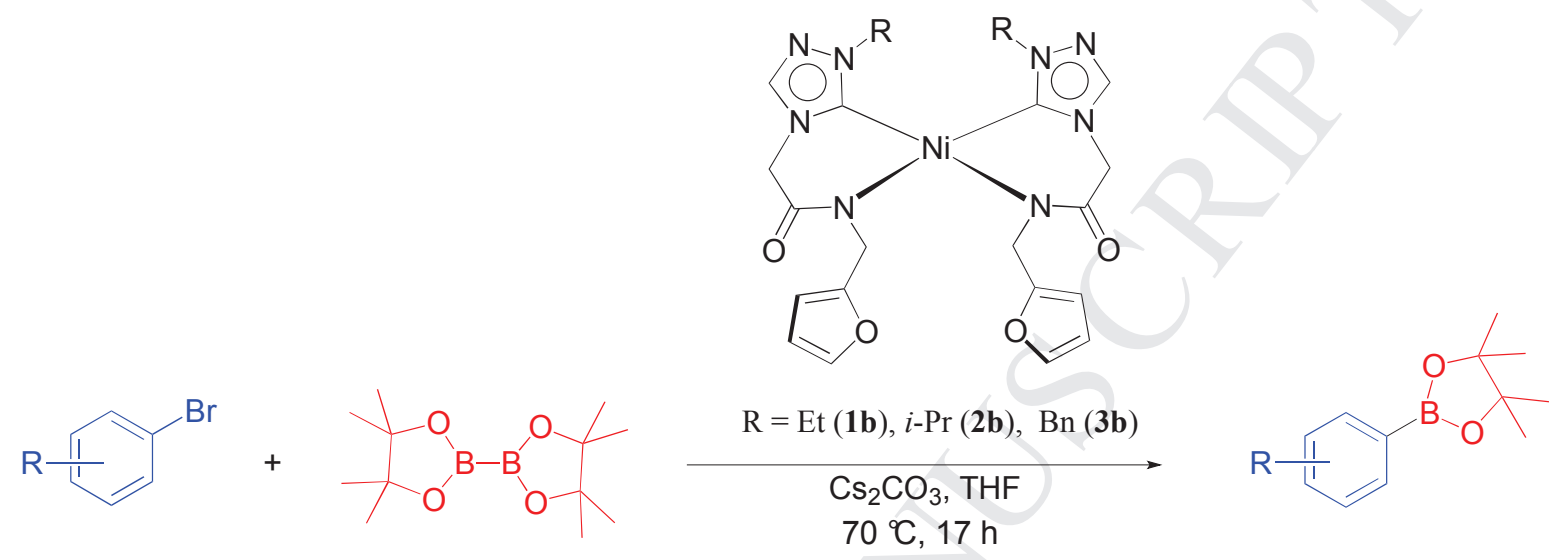

A series of nickel complexes of 1,2,4-triazole derived amido-functionalized N-heterocyclic carbene ligands were prepared, characterized and explored for their activity in the borylation reaction of arylbromides. 


\section{References}

[ 1 ]. (a). A.P. Prakasham, P. Ghosh, Inorg. Chim. Acta (2014) http://dx.doi.org/10.1016/j.ica.2014.11.005

(b). S.Z. Tasker, E.A. Standley, T.F. Jamison, Nature 509 (2014) 299-309.

(c). S.Wang, W.H. Sun, C. Redshaw, J. Organomet. Chem. 751 (2014) 717-741.

(d). R. Gao, W.H. Sun, C. Redshaw, Catal. Sci. Technol. 3 (2013) 1172-1179.

(e). A. Correa, J. Cornella, R. Martin, Angew. Chem. Int. Ed. 52 (2013) 1878-1880.

(f). J. Yamaguchi, K. Muto, K. Itami, Eur. J. Org. Chem. (2013) 19-30.

[2]. (a). R. Chinchilla, C. Nájera, Chem. Soc. Rev. 114 (2014) 1783-1826.

(b). C. Valente, M. Pompeo, M. Sayah, M.G. Organ, Org. Proc. Res. Dev. 18 (2014) 180-190.

(c). Q.A. Chen, Z.S. Ye, Y. Duan, Y.G. Zhou, Chem. Soc. Rev. 42 (2013) 497-511.

(d). S.I. Gorelsky, Coord. Chem. Rev. 257 (2013)153-164.

(e). T. Vlaar, E. Ruijter, B.U.W. Maes, R.V.A. Orru, Angew. Chem. Int. Ed. 52 (2013) 7084-7097.

(f). K. Muñiz, C. Martínez, J. Org. Chem. 78 (2013) 2168-2174.

[3]. G.A. Foulds, Coord. Chem. Rev. 146 (1995) 1-90.

[4]. A. Arévalo, J.J. García, Eur. J. Inorg. Chem. (2010) 4063-4074..

[5]. (a). A. Kumar, P. Ghosh, Eur. J. Inorg. Chem. (2012) 3955-3969.

(b). A. John, P. Ghosh, Dalton Trans. 39 (2010) 7183-7206.

[6]. (a). C.C. J. Loh, D. Enders, Chem. Eur. J. 18 (2012) 10212-10225.

(b). T. Kçsterke, J. Kçsters, E-Ulrich Würthwein, C. Mück-Lichtenfeld, C. S. Brinke, F. Lahoz, F. E. Hahn, Chem. Eur. J. 18 (2012) 14594-14598. 
(c). T. Kösterke, T. Pape, F. E. Hahn, Chem. Commun. 47 (2011) 10773-10775.

(d). G.C. Fortman, S.P. Nolan, Chem. Soc. Rev. 40 (2011) 5151-5169.

(e). C. Radloff, F. E. Hahn, T. Pape, R. Fröhlich, Dalton Trans. (2009) 7215-7222.

(f). F. E. Hahn, C. Radloff, T. Pape, A. Hepp, Organometallics 27 (2008) 6408-6410.

(g). F. Boeda, S.P. Nolan, Annu. Rep. Prog. Chem. Sect. B 104 (2008) 184-210.

(h). N. Marion, S.P. Nolan, Chem. Soc. Rev. 37 (2008) 1776-1782.

(i). F.E. Hahn, B. Heidrich, A. Hepp, T. Pape, J. Organomet. Chem. 692 (2007) $4630-4638$.

(j). H. V. Huynh, C. Holtgrewe, T. Pape, L. L. Koh, E. Hahn, Organometallics 25 (2006) 245-249 .

(k). R. Singh, S.P. Nolan, Annu. Rep. Prog. Chem. Sect. B 102 (2006) 168-196.

(1). T. Weskamp, V.P.W. Böhm, W.A. Herrmann, J. Organomet. Chem. 600 (2000) $12-22$.

[7]. C. Dash, M.M. Shaikh, R.J. Butcher, P. Ghosh, Dalton Trans. 39 (2010) 25152524.

[8]. (a). S. Kumar, A. Narayanan, M.N. Rao, M.M. Shaikh, P. Ghosh, J. Organomet. Chem. 696 (2012) 4159-4165.

(b). M.K. Samantaray, M.M. Shaikh, P. Ghosh, Organometallics 28 (2009) 22672275.

(c). S. Ray, M.M. Shaikh, P. Ghosh, Eur. J. Inorg. Chem. (2009) 1932-1941.

[9 ]. M. Katari, G. Rajaraman, and P. Ghosh, J. Organomet. Chem. (2014) http://dx.doi.org/10.1016/j.jorganchem.2014.02.012.

[10]. (a). L.P. Bheeter, M. Henrion, L. Brelot, C. Darcel, M.J. Chetcuti, J.-B. Sortais, V. Ritleng, Adv. Synth. Catal. 354 (2012) 2619-2624. 
(b). L.P. Bheeter, M. Henrion, M.J. Chetcuti, C. Darcel, V. Ritleng, J.-B. Sortais, Catal. Sci. Technol. 3 (2013) 3111-3116.

[11]. (a). P. Li, C. Fu, S. Ma, Org. Biomol. Chem. 12 (2014) 3604-3610.

(b). W.K. Chow, O.Y. Yuen, P.Y. Choy, C.M. So, C.P. Lau, W.T. Wong, F.Y. Kwong, RSC Adv. 3 (2013) 12518-12539.

(c). J. Takaya, N. Iwasawa, ACS Catal. 2 (2012) 1993-2006.

(d). M.J. Ingleson, Synlett 23 (2012) 1411-1415.

(e). J.F. Hartwig, Chem. Soc. Rev. 40 (2011) 1992-2002.

[12]. (a). R.J.P. Corriu, J.P. Masse, J. Chem. Soc. Chem. Commun. (1972) 144.

(b). K. Tamao, K. Sumitani, M. Kumada, J. Am. Chem. Soc. 94 (1972) 4374-4376.

[13]. For a representative review, see, F.-S. Han, Chem. Soc. Rev. 42 (2013) 52705298.

For representative recent examples, see,

(a). S.D. Ramgren, L. Hie, Y. Ye, N.K. Garg, Org. Lett. 15 (2013) 3950-3953.

(b). A.M. Oertel, V. Ritleng, M.J. Chetcuti, Organometallics 31 (2012) 2829-2840.

(c). Y.-L. Zhao, Y. Li, S.-M. Li, Y.-G. Zhou, F.-Y. Sun, L.-X. Gao, F.-S. Han, Adv. Synth. Cat. 353 (2011) 1543-1550.

(d). S. Ge, J.F. Hartwig, Angew. Chem. Int. Ed. 51 (2012) 12837-12841.

(e). V. Ritleng, A.M. Oertel, M.J. Chetcuti, Dalton Trans. 39 (2010) 8153-8160.

(f). K. Inamoto, J.-i. Kuroda, E. Kwon, K. Hiroya, T. Doi, J. Organomet. Chem. 694 (2009) 389-396.

(g). J.-i. Kuroda, K. Inamoto, K. Hiroya, T. Doi, Eur. J. Org. Chem. (2009) 22512261.

(h). Y. Zhou, Z. Xi, W. Chen, D. Wang, Organometallics 27 (2008) 5911-5920. 
(i). Z. Xi, X. Zhang, W. Chen, S. Fu, D. Wang, Organometallics 26 (2007) 66366642.

(j). C.-Y. Liao, K.-T. Chan, Y.-C. Chang, C.-Y. Chen, C.-Y. Tu, C.-H. Hu, H.M. Lee, Organometallics 26 (2007) 5826-5833.

(k). C.-C. Lee, W.-C. Ke, K.-T. Chan, C.-L. Lai, C.-H. Hu, H.M. Lee, Chem. Eur. J.

13 (2007) 582-591.

(1). K. Inamoto, J.-i. Kuroda, T. Sakamoto, K. Hiroya, Synthesis (2007) 2853-2861.

(m). P.L. Chiu, C.-L. Lai, C.-F. Chang, C.-H. Hu, H.M. Lee, Organometallics 24 (2005) 6169-6178.

(n). D.S. McGuinness, K.J. Cavell, B.W. Skelton, A.H. White, Organometallics 18 (1999) 1596-1605.

[14]. For selected recent examples, see,

(a). P. Leowanawat, A.-M. Resmerita, C. Moldoveanu, C. Liu, N. Zhang, D.A.

Wilson, L.M. Hoang, B.M. Rosen, V. Percec, J. Org. Chem. 75 (2010) 7822-7828.

(b). T. Yamamoto, T. Morita, J. Takagi, T. Yamakawa, Org. Lett. 13 (2011) 57665769.

(c). J. Yi, J.-H. Liu, J. Liang, J.-J. Dai, C.-T. Yang, Y. Fu, L. Liu, Adv. Synth. Catal. 354 (2012) 1685-1691.

(d). M. Murata, Y. Sogabe, T. Namikoshi, S. Watanabe, Heterocycles 86 (2012) 133138.

(e). G.A. Molander, L.N. Cavalcanti, C. Garcia-Garcia, J. Org. Chem. 78 (2013) $6427-6439$.

(f). A.S. Dudnik, G.C. Fu, J. Am. Chem. Soc. 134 (2012) 10693-10697. 
[15]. For example, the literature searchable by Scifinder Scholar provided 55 number of references for the transition metal complexes of any amido-functionalized imidazole based N-heterocyclic carbene (NHC) ligands while the similar search for the transition metal complexes of any amido-functionalized 1,2,4 triazole based Nheterocyclic carbene (NHC) ligands provided only 1 reference. See, SciFinder Scholar. https://scifinder.cas.org. Accessed August 30 (2014).

[16]. Analogous search in the Cambridge Structural database for the structurally characterized transition metal complexes likewise yielded 31 references for the amido-functionalized imidazole based N-heterocyclic carbene (NHC) complexes and only 1 reference for the transition metal the amido-functionalized 1,2,4 triazole based N-heterocyclic carbene (NHC) complexes. See, Cambridge Structural database Version 5.35, November 2013.

[17]. Cambridge Structural database Version 5.35, November (2013).

[18]. P.G. Bulger, I.F. Cottrell, C.J. Cowden, A.J. Davies, U-H. Dolling, Tetrahedron Lett. 41 (2000) 1297-1301.

[19]. F. Dallacker, K. Minn, Chemiker-Zeitung 110 (1986) 101-108.

[20]. B.A. Astleford, G.L. Goe, J.G. Keay, E.F.V. Scriven, J. Org.Chem, 54 (1989) $731-732$.

[21]. M. Bayrakcı, Ş. Yiğiter, Tetrahedron 69 (2013) 3218-3224.

[22]. Gaussian 09, Revision A.1, M.J. Frisch, G.W. Trucks, H.B. Schlegel, G.E. Scuseria, M.A. Robb, J.R. Cheeseman, G. Scalmani, V. Barone, B. Mennucci, G.A. Petersson, H. Nakatsuji, M. Caricato, X. Li, H.P. Hratchian, A.F. Izmaylov, J. Bloino, G. Zheng, J.L. Sonnenberg, M. Hada, M. Ehara, K. Toyota, R. Fukuda, J. Hasegawa, M. Ishida, T. Nakajima, Y. Honda, O. Kitao, H. Nakai, T. Vreven, J.A. Montgomery, 
J.E. Peralta, F. Ogliaro, M. Bearpark, J.J. Heyd, E. Brothers, K.N. Kudin, V.N.

Staroverov, R. Kobayashi, J. Normand, K. Raghavachari, A. Rendell, J.C. Burant, S.S. Iyengar, J. Tomasi, M. Cossi, N. Rega, J.M. Millam, M. Klene, J.E. Knox, J.B. Cross, V. Bakken, C. Adamo, J. Jaramillo, R. Gomperts, R.E. Stratmann, O. Yazyev, A.J. Austin, R. Cammi, C. Pomelli, J.W. Ochterski, R.L. Martin, K. Morokuma, V.G. Zakrzewski, G. A. Voth, P. Salvador, J.J. Dannenberg, S. Dapprich, A.D. Daniels, O. Farkas, J. B. Foresman, J.V. Ortiz, J. Cioslowski, D.J. Fox, Gaussian, Inc., Wallingford CT, (2009).

[23]. (a). A.D. Becke, Phys. Rev. A 38 (1998) 3098-3100.

(b). C. Lee, W. Yang, R.G. Parr, Phys. Rev. B 37 (1998) 785-789.

[24 ]. (a). W.J. Herre, R. Dditcrfield, J.A. Pople, J. Chem. Phys. 56 (1972) $2257-2261$.

(b). G.A. Petersson, A. Bennett, T.G. Tensfeldt, M.A. AI-Laham, W.A. Shirley, J. Chem. Phys. 89 (1988) 2193-2218.

(c). G. A. Petersson, M. A. AI-Laham, J. Chem. Phys. 94 (1991) 6081-6090.

[25]. (a). O. Das, N.N. Adarsh, A. Paul, T.K. Paine, Inorg. Chem. 49 (2010) 541551.

(b). O. Gutierrez, D.J. Tantillo, Organometallics 29 (2010) 3541-3545.

(c). M. Ishida, Y. Naruta, F. Tani, Dalton Trans. 39 (2010) 2651-2659.

(d). E.B. Kadossov, K.J. Gaskell, M.A. Langell, J. Comput. Chem. 28 (2007) $1240-1251$.

(e). D. C. Graham, K. J. Cavell, B. F. Yates, Dalton Trans. (2007) 4650-4658.

(f). Q.Z. Han, Y.H. Zhao, H. Wen, Data Sci. J. 6 (2007) S837-S846.

[26]. A.E. Reed, L.A. Curtiss, F. Weinhold, Chem. Rev. 88 (1988) 899-926. 
[27]. S. Dapprich, G. Frenking, J. Phys. Chem. 99 (1995) 9352-9362.

[28]. (a). D. Nemcsok, K. Wichmann, G. Frenking, Organometallics 23 (2004) $3640-3646$.

(b). S.F. Vyboishchikov, G. Frenking, Chem. Eur. J. 4 (1998) 1439-1448.

(c). C. Boehme, G. Frenking, Organometallics 17 (1998) 5801-5809.

(d). G. Frenking, U. Pidun, J. Chem. Soc. Dalton Trans. (1997) 1653-1662.

[29]. S.I. Gorelsky, AOMix: Program for Molecular Orbital Analysis, version 6.87d; University of Ottawa: Ottawa, Canada, 2014.; http://www.sg-chem.net/ (accessed June 18, 2014).

[30]. (a). S.I. Gorelsky, J. Chem. Theory Comput., 8 (2012) 908-914.

(b). D.R. Denomme, S.M. Dumbris, I.F.D. Hyatt, K.A. Abboud, I. Ghiviriga, L. McElwee-White, Organometallics 29 (2010) 5252-5256.

(c). N. Dimakis, M. Cowan, G. Hanson, E. S. Smotkin, J. Phys. Chem. C 113 (2009) 18730-18739.

(d). S.I. Gorelsky, E.I. Solomon, Theor. Chem. Acc. 119 (2008) 57-65.

(e). S.I. Gorelsky, A.B.P. Lever, J. Organomet. Chem. 635 (2001) 187-196.

[31]. (a). S.I. Gorelsky, S. Ghosh, E.I. Solomon, J. Am. Chem. Soc. 128 (2006) 278-290.

(b). S.I. Gorelsky, L. Basumallick, J. Vura-Weis, R. Sarangi, K.O. Hodgson, B. Hedman, K. Fujisawa, E.I. Solomon, Inorg. Chem. 44 (2005) 4947-4960. 
Figure 1. The nickel complexes of 1,2,4-triazole derived amido-functionalized Nheterocyclic carbene Ligands. 
Highlights

- Ni complexes of the 1,2,4-triazole based NHC ligands were synthesized.

- The Ni-NHC interaction was probed by DFT studies

- The Ni-NHC complexes exhibited moderate activity in borylation of aryl bromides. 


\section{Supporting Information}

\section{Nickel Complexes of 1,2,4-Triazole Derived Amido-functionalized N- heterocyclic Carbene Ligands: Synthesis, Theoretical Studies and Catalytic Application}

Anuj Kumar, ${ }^{\dagger}$ Linus Paulin Bheeter, ${ }^{\star}$ Manoj Kumar Gangwar, ${ }^{\dagger}$ Jean-Baptiste Sortais, ${ }^{\star}$ Christophe Darcel ${ }^{*}$, and Prasenjit Ghosh ${ }^{*, \dagger}$

${ }^{\dagger}$ Department of Chemistry

Indian Institute of Technology Bombay, Powai, Mumbai 400076.

tUniversité de Rennes 1, UMR CNRS-UR1 6226 Institut des Sciences chimiques de Rennes, Team "Organometallics: Matrials and Catalysis" - Centre for Catalysis and Green Chemistry

Campus de Beaulieu, 35042 - Rennes, France

Email: pghosh@chem.iitb.ac.in, christophe.darcel@univ-rennes1.fr,

Fax: +9122 $25723480,+33223236939$ 


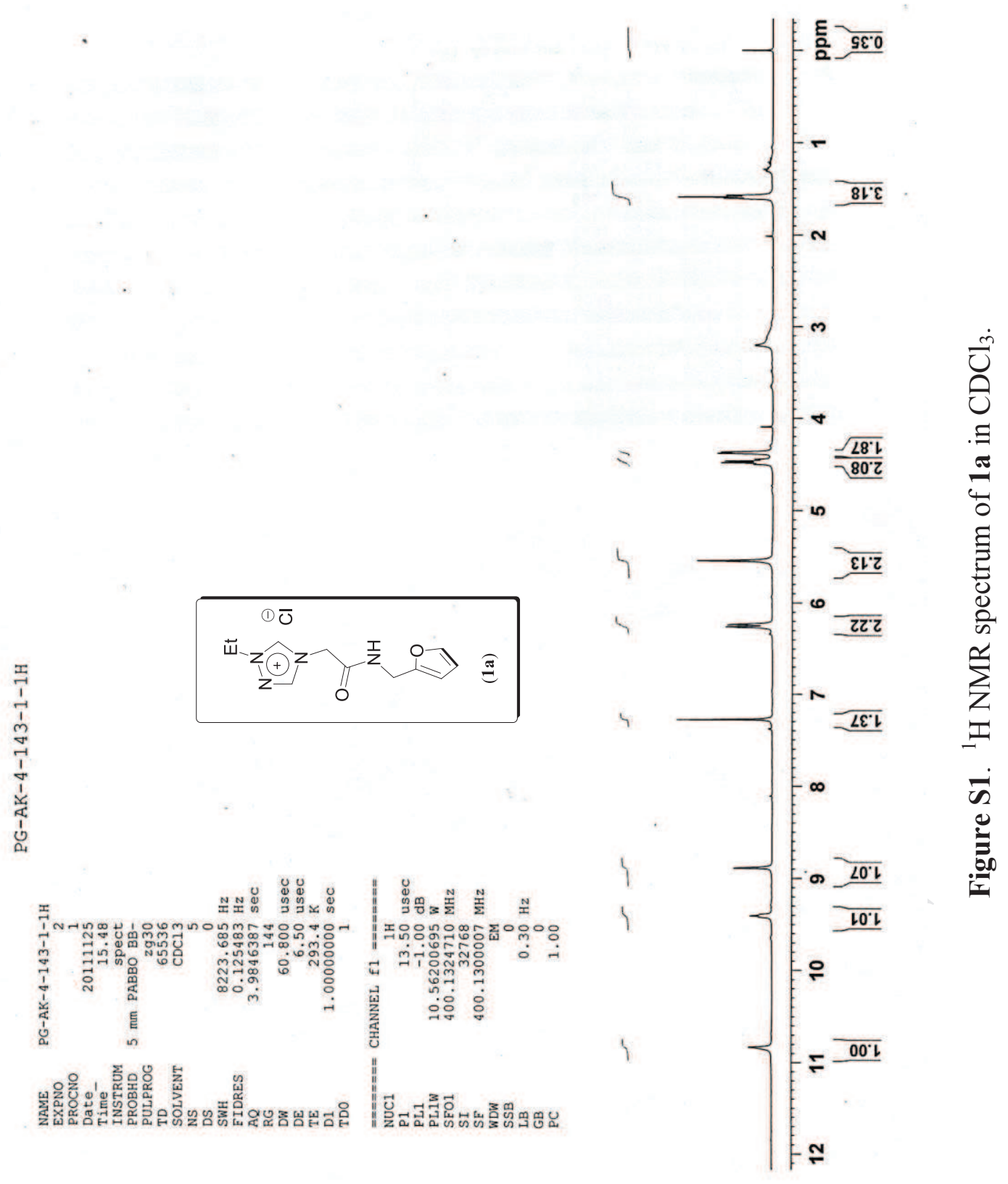




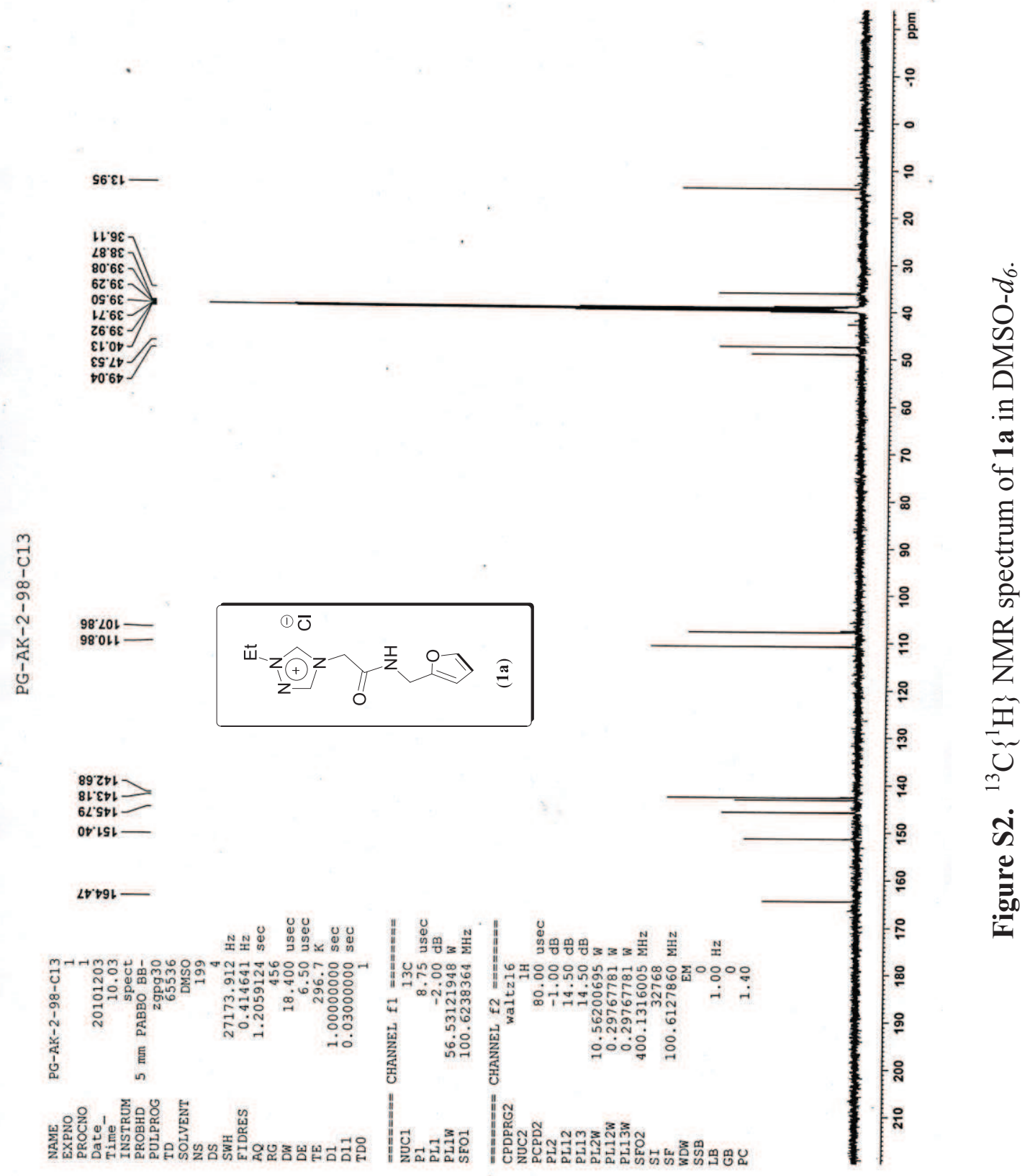




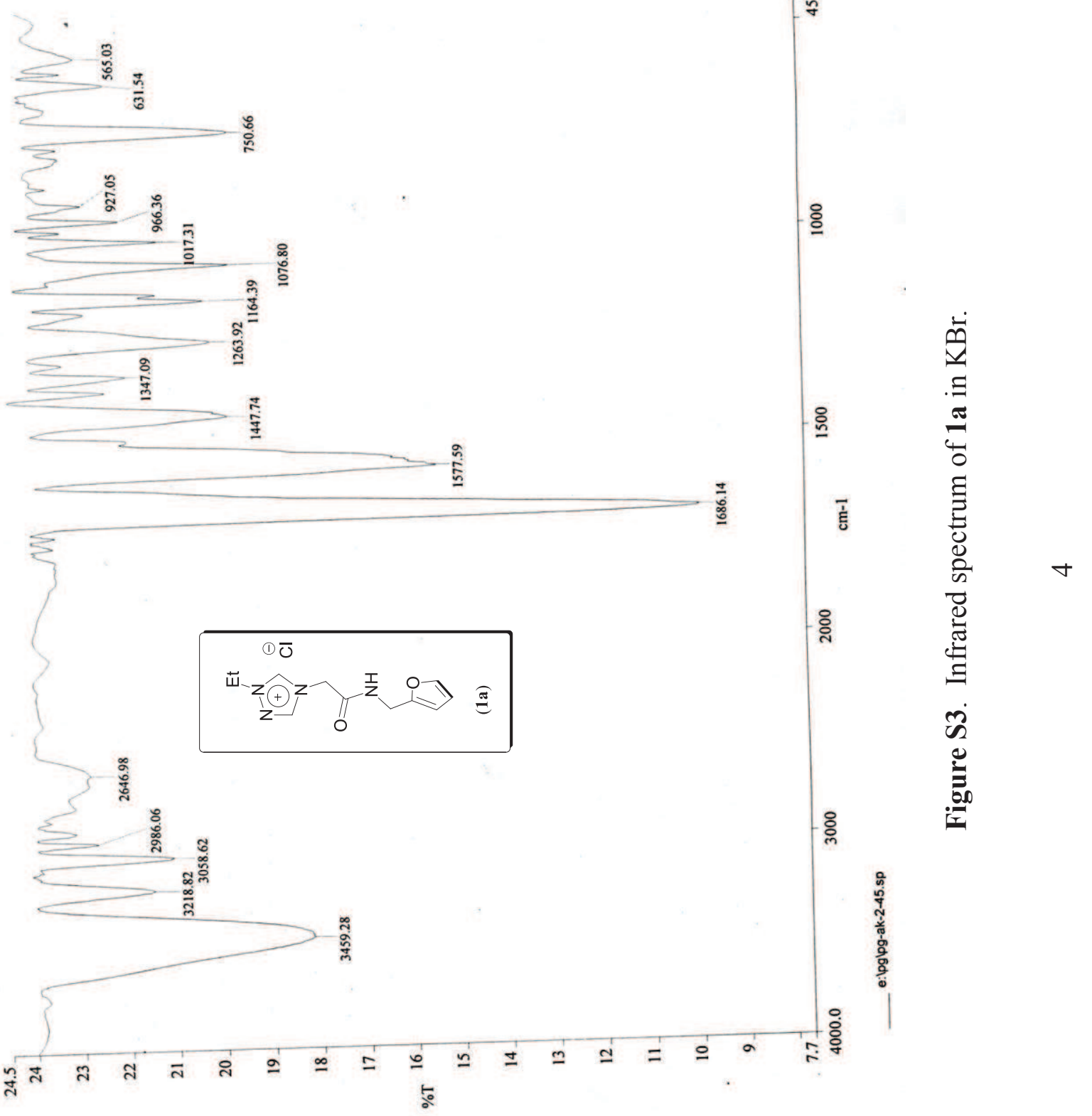




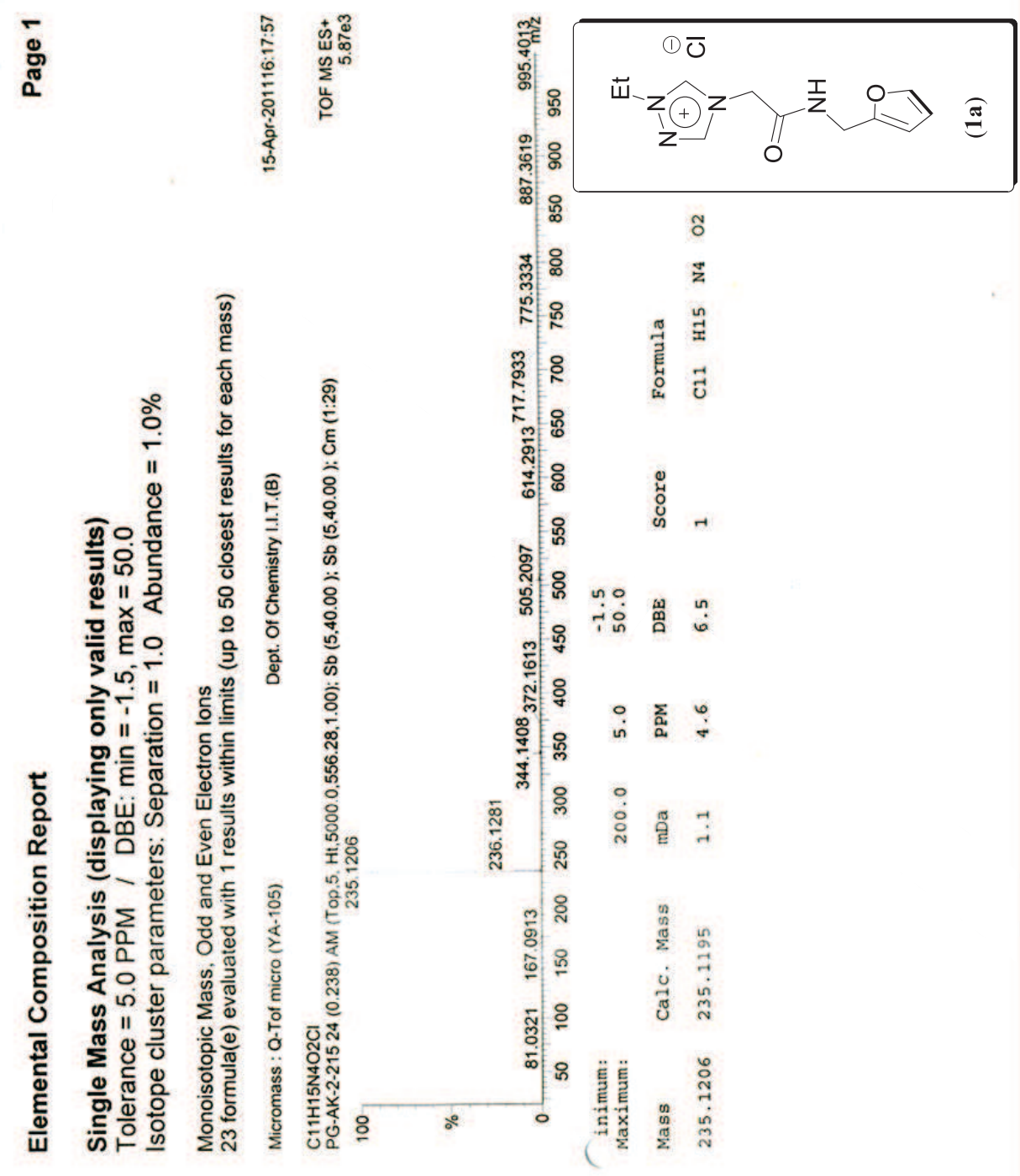

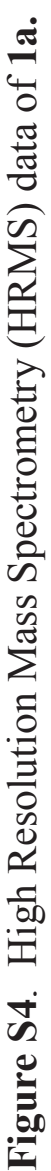




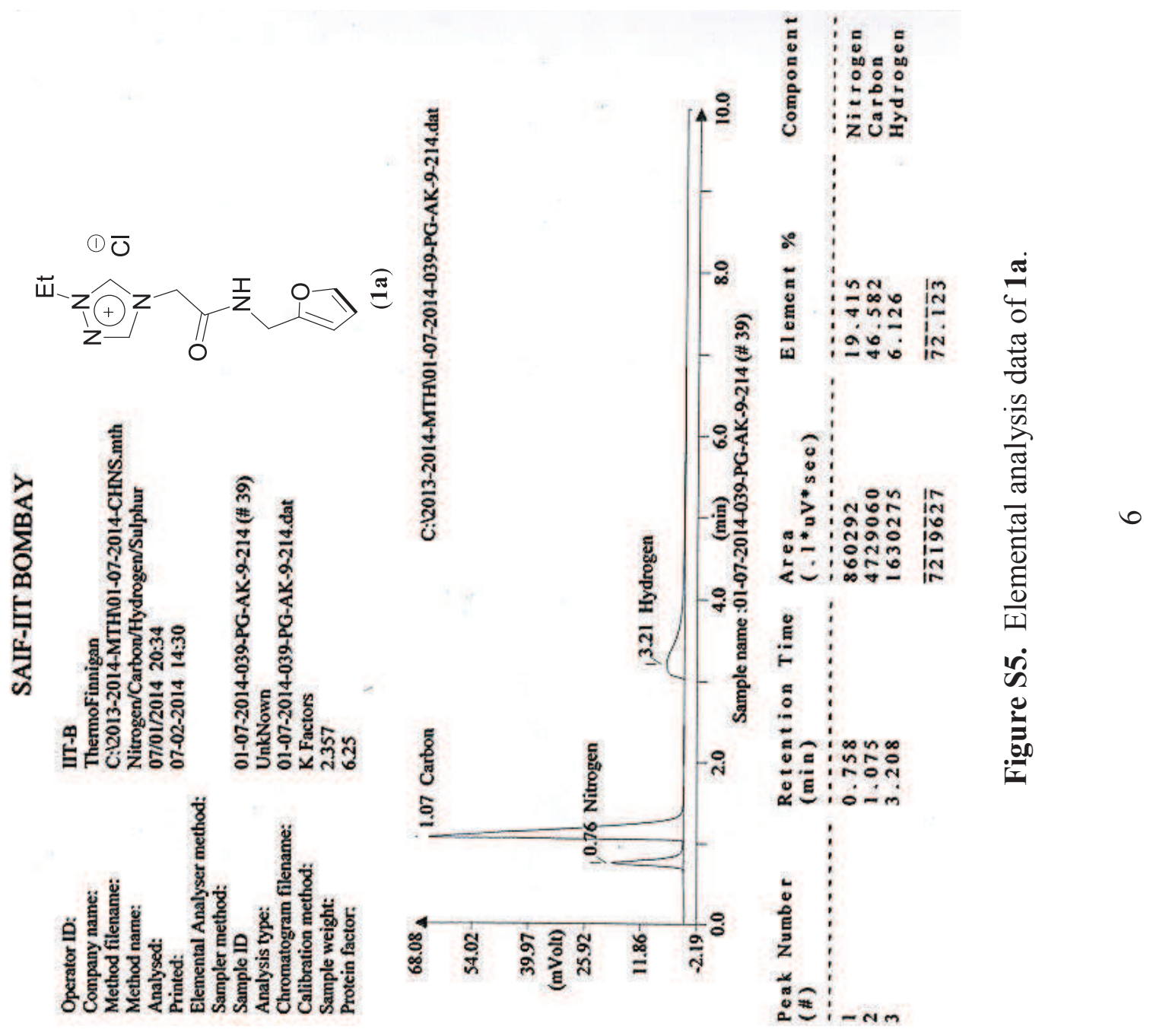




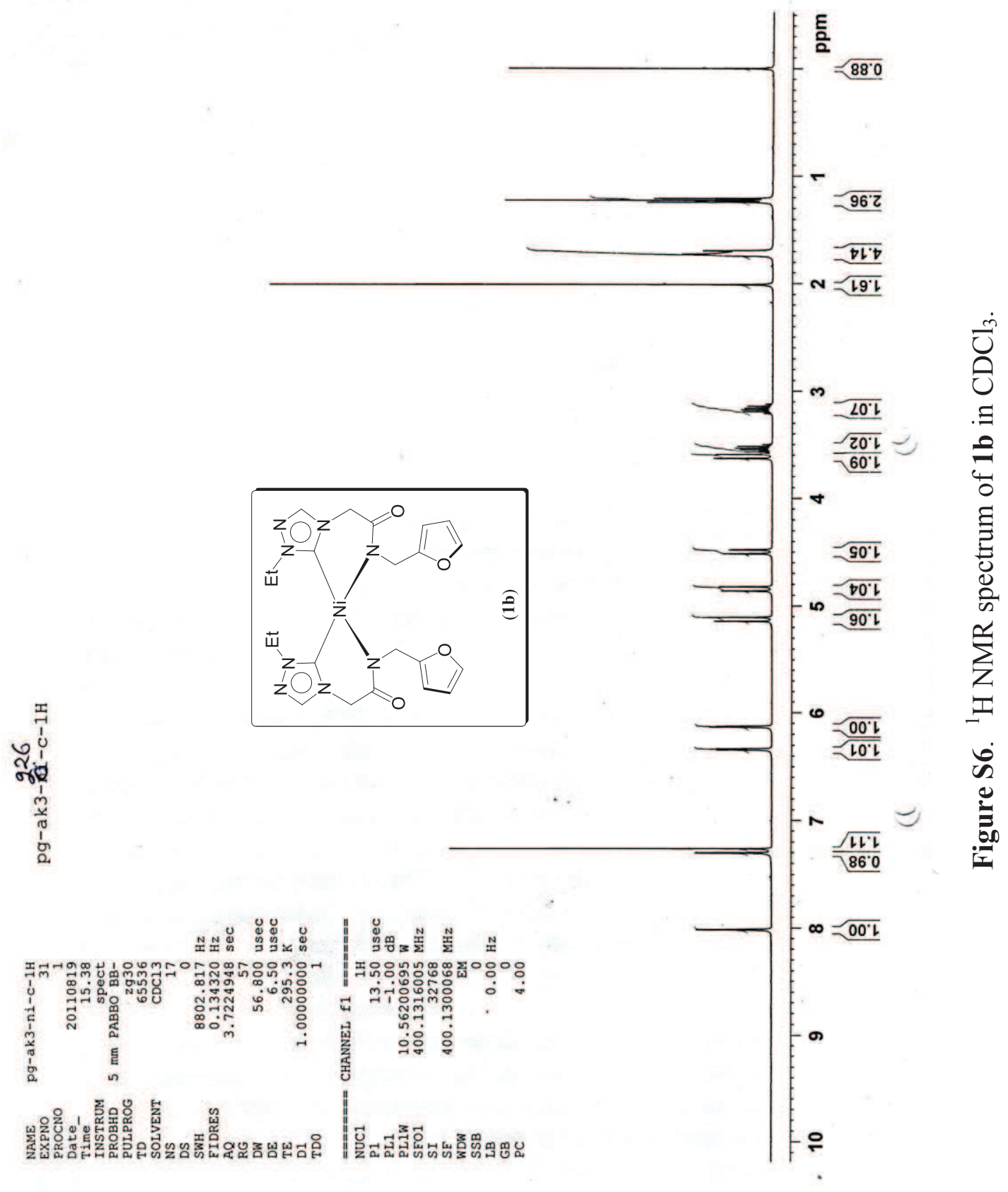




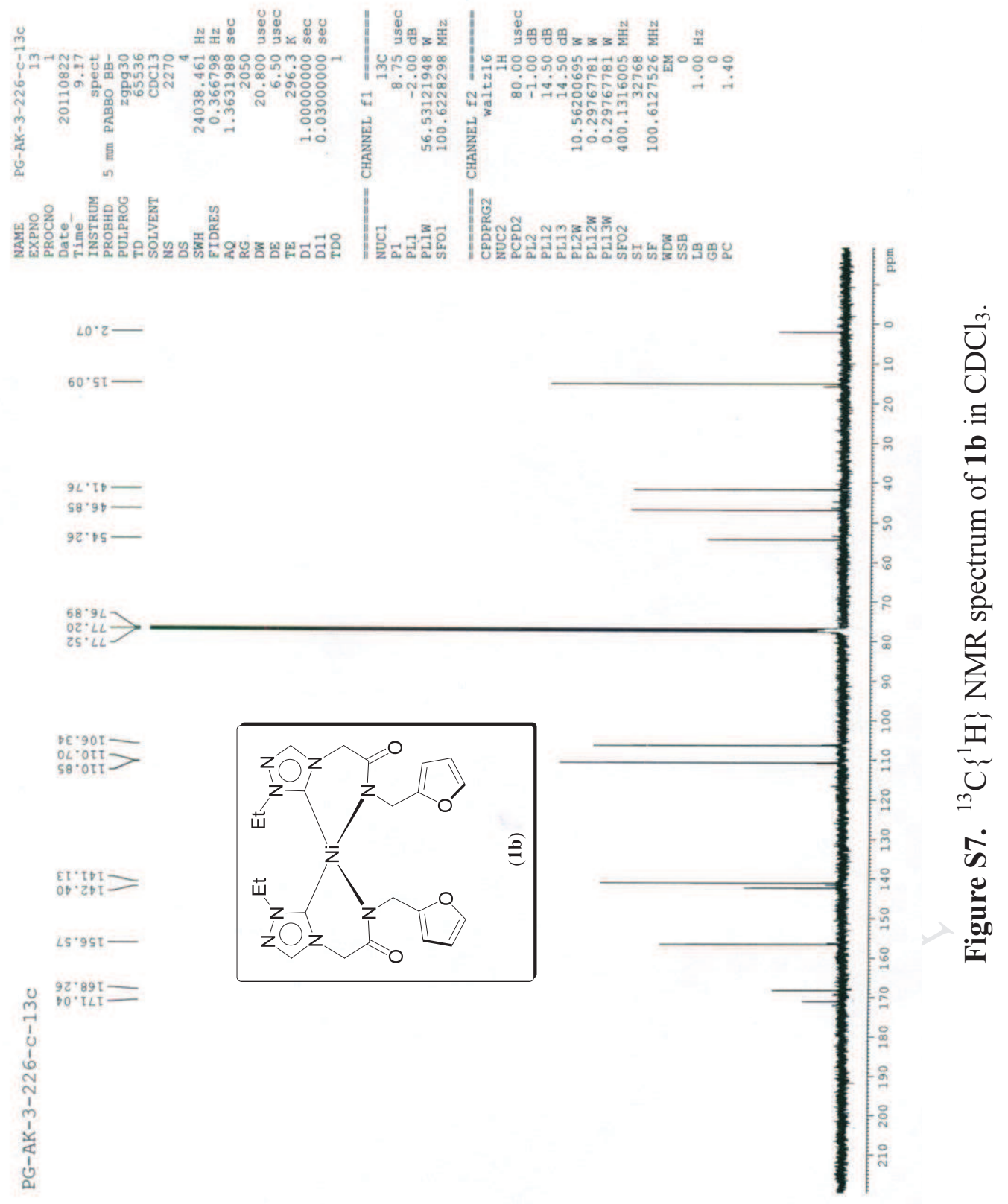




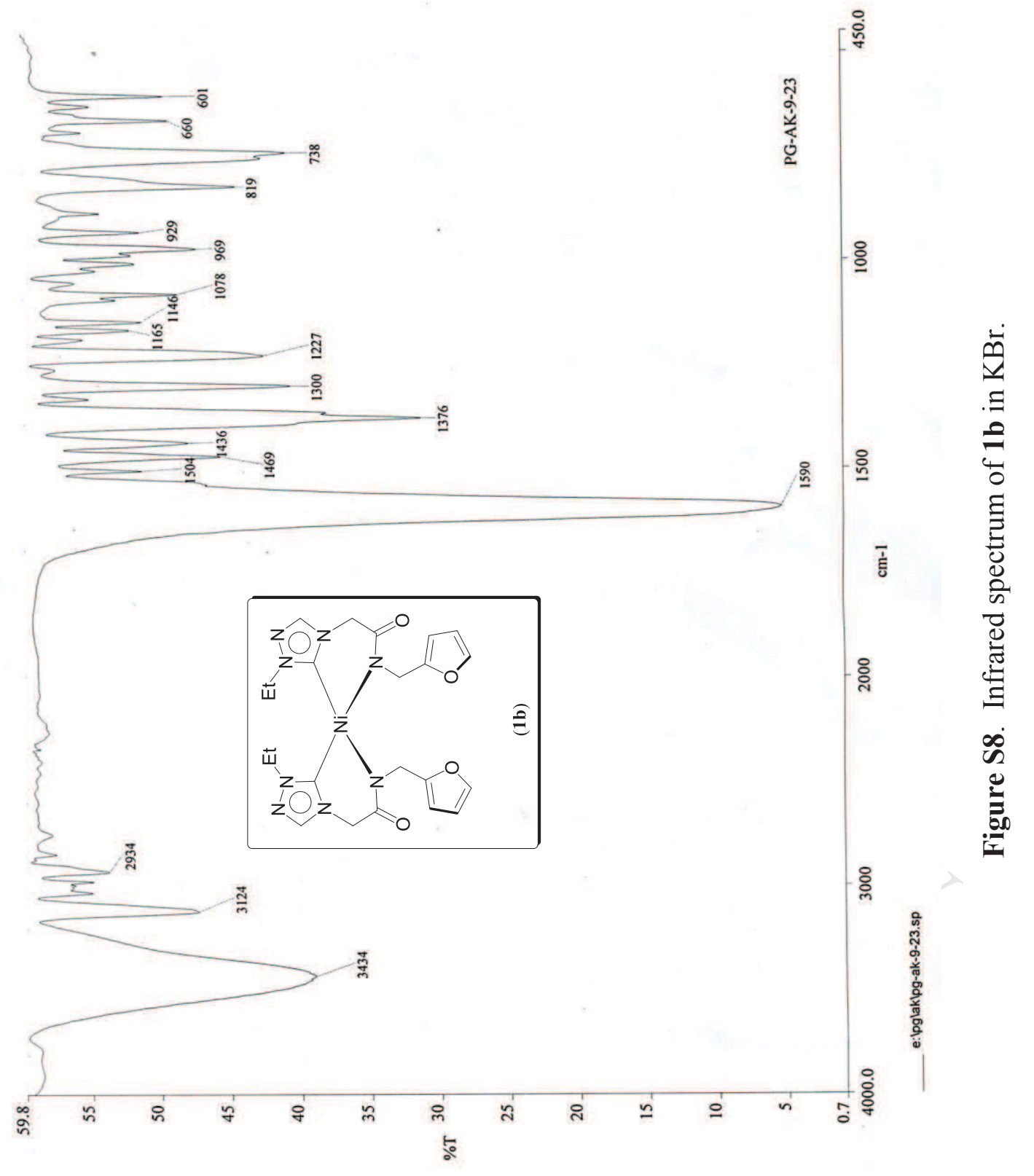




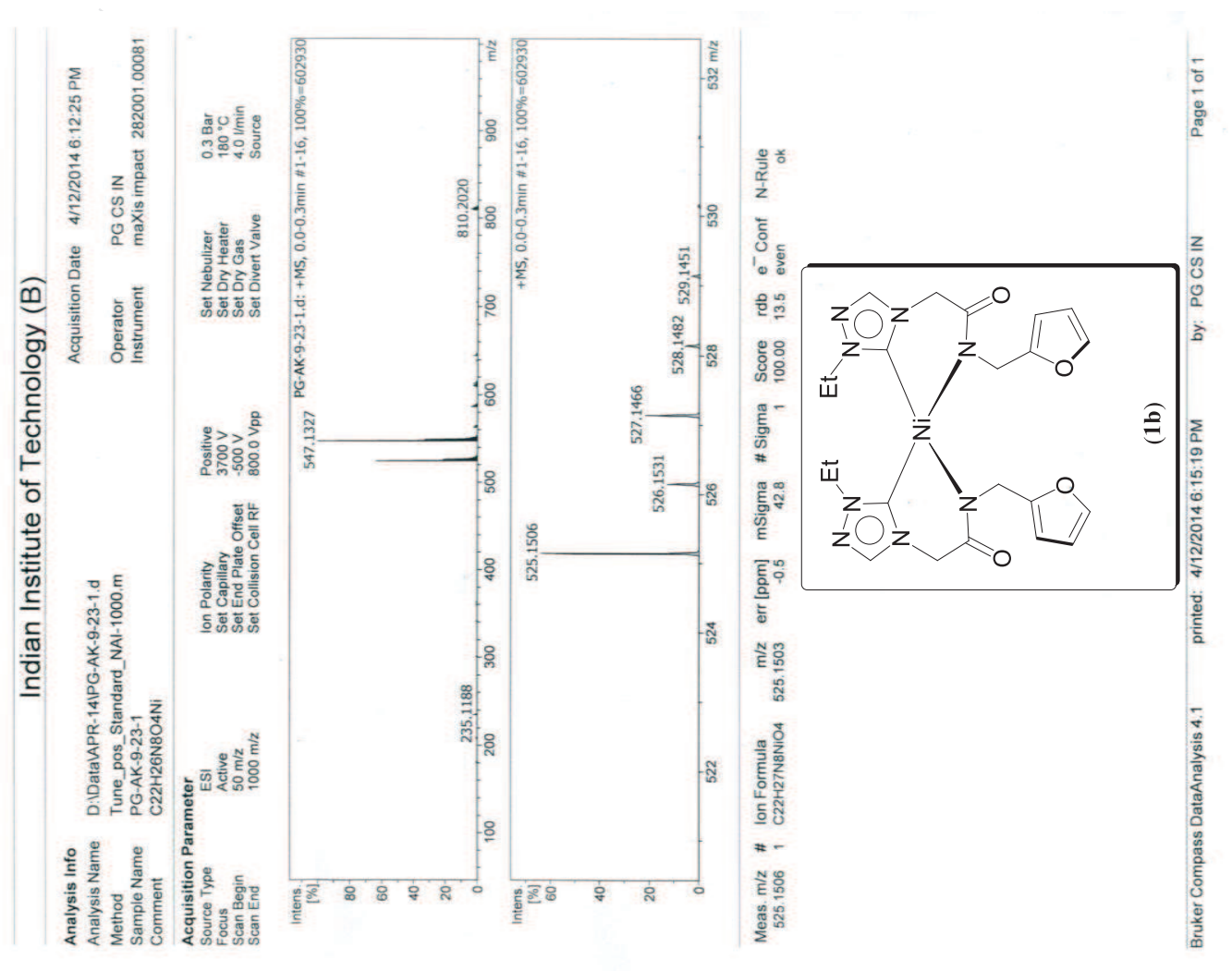

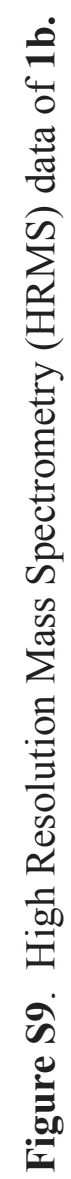




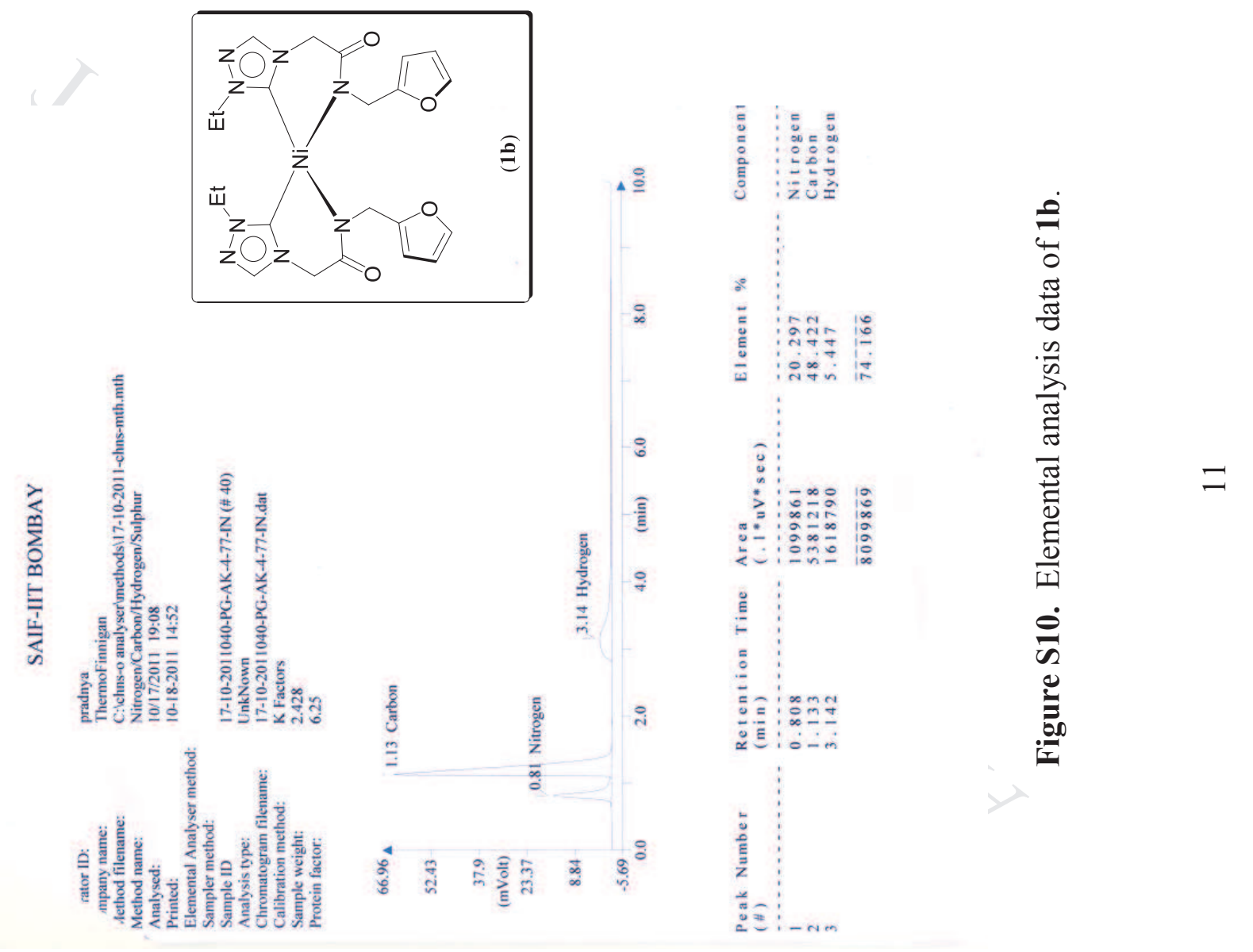




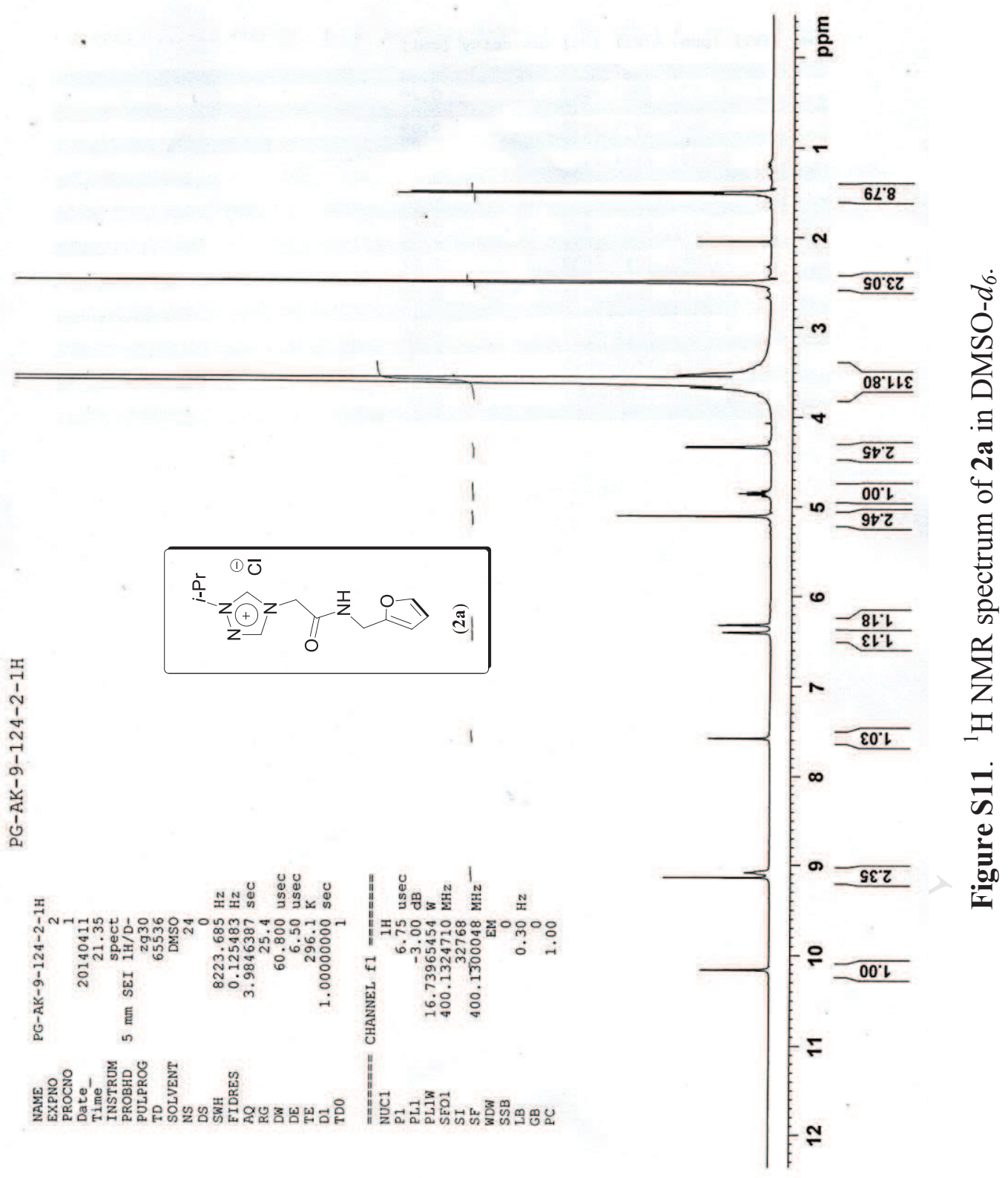




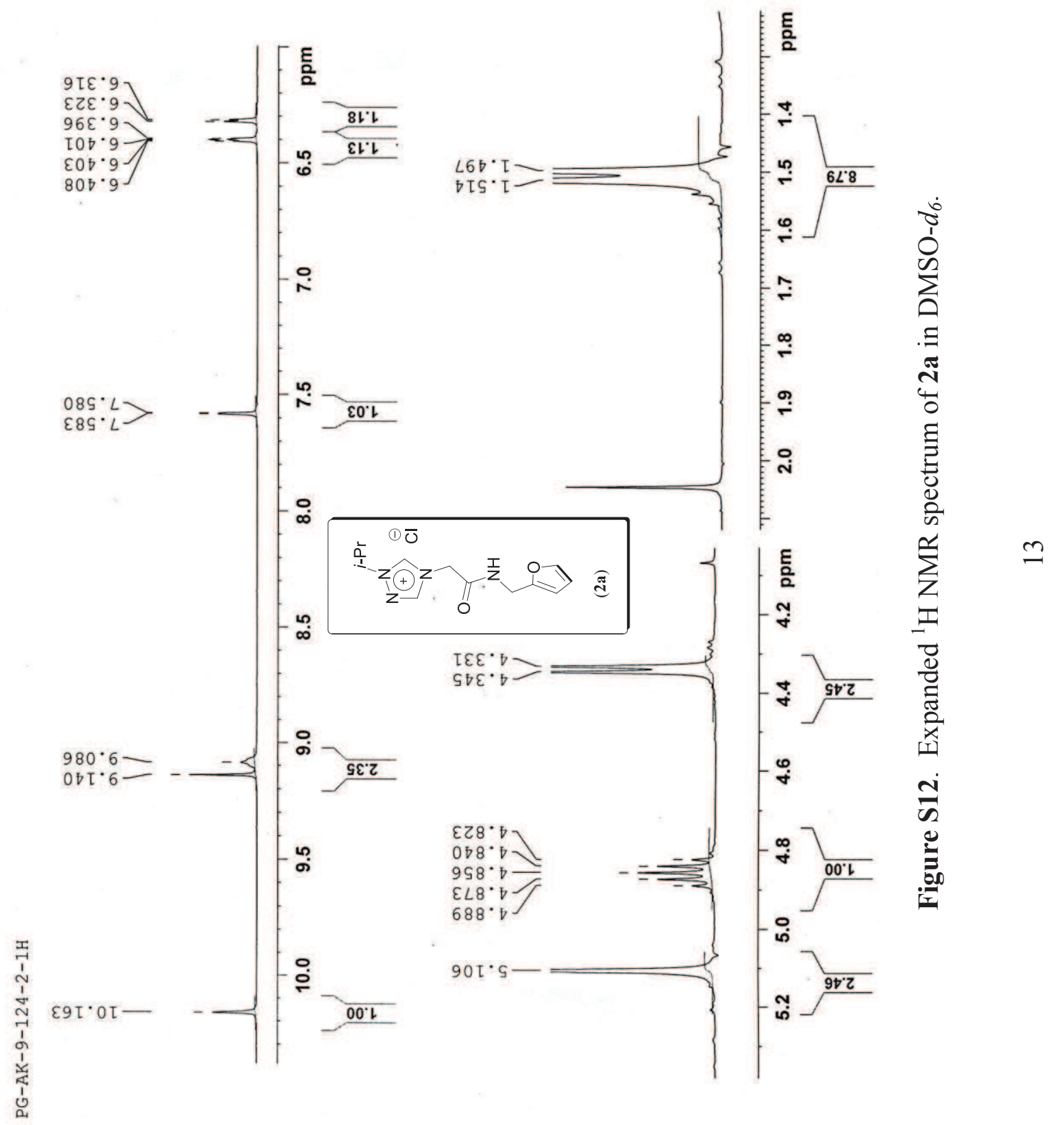




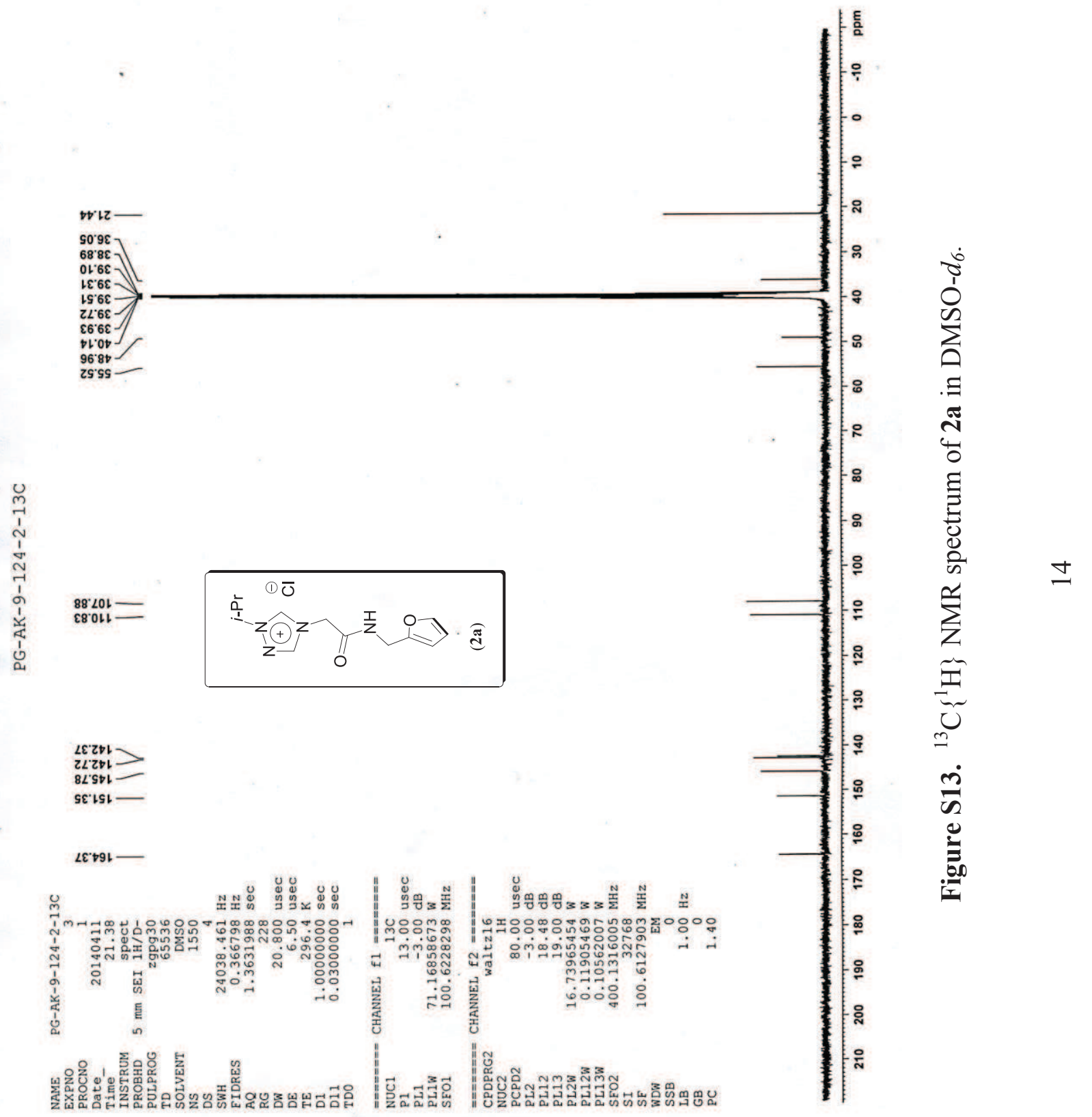




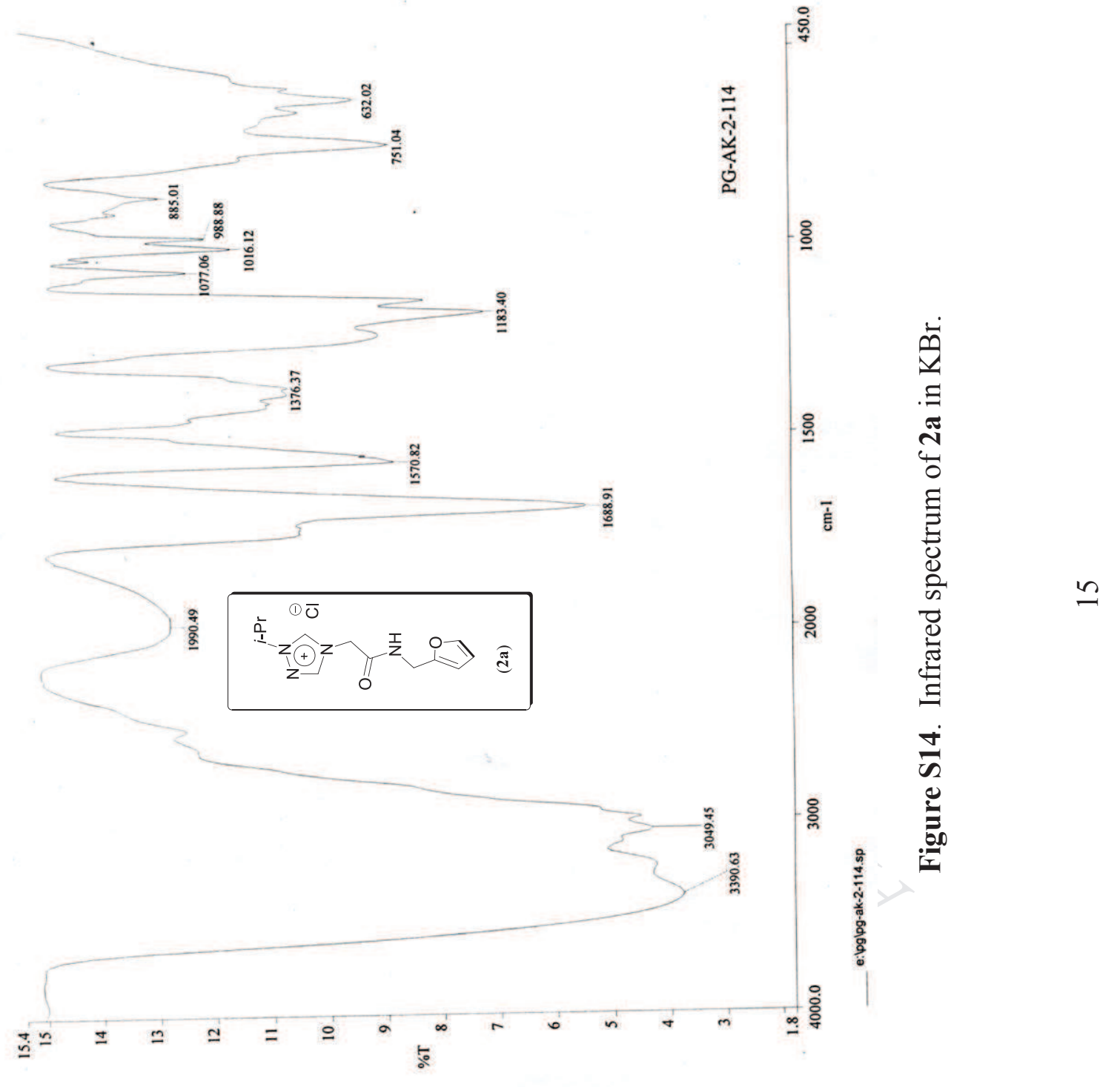




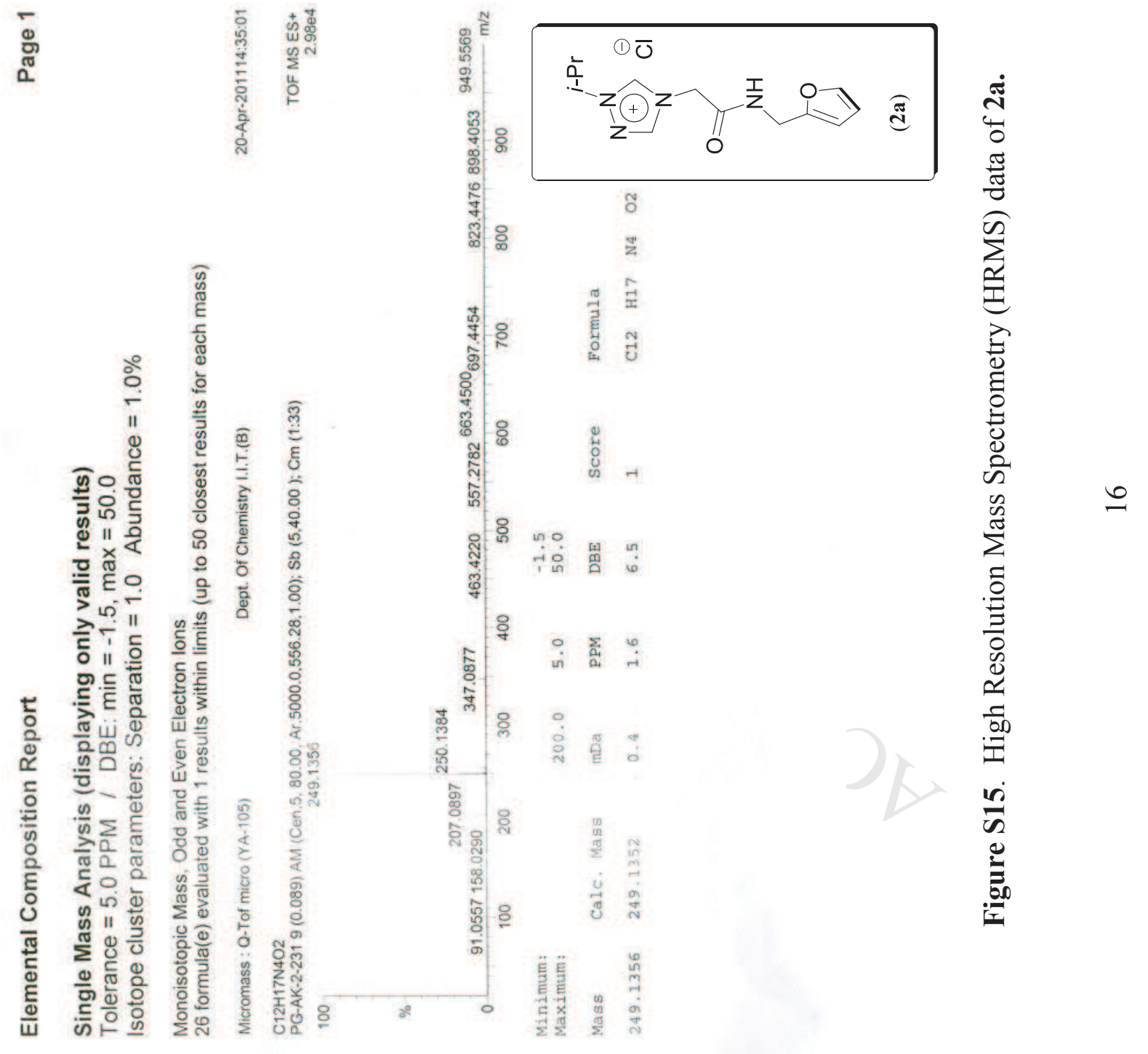




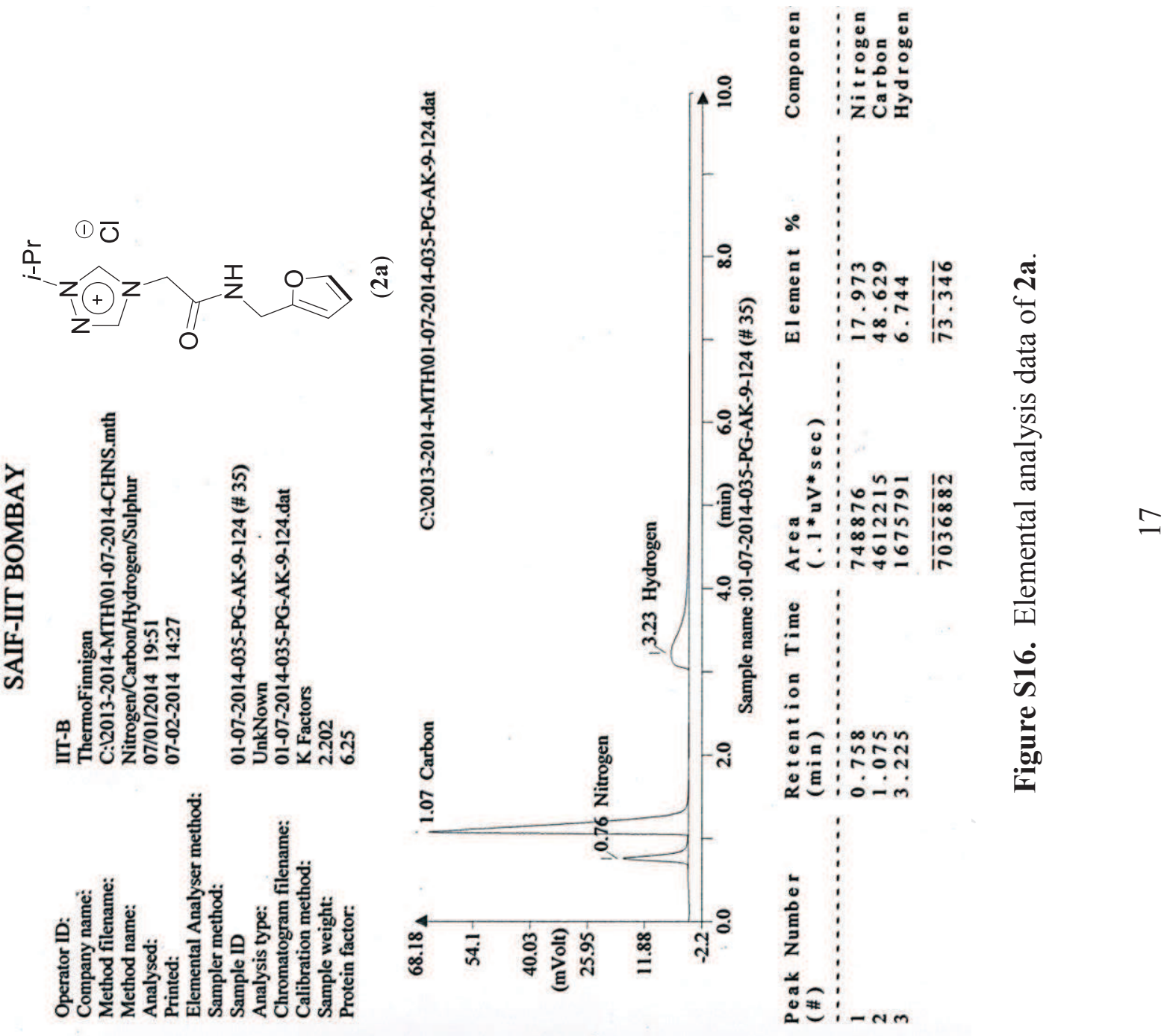




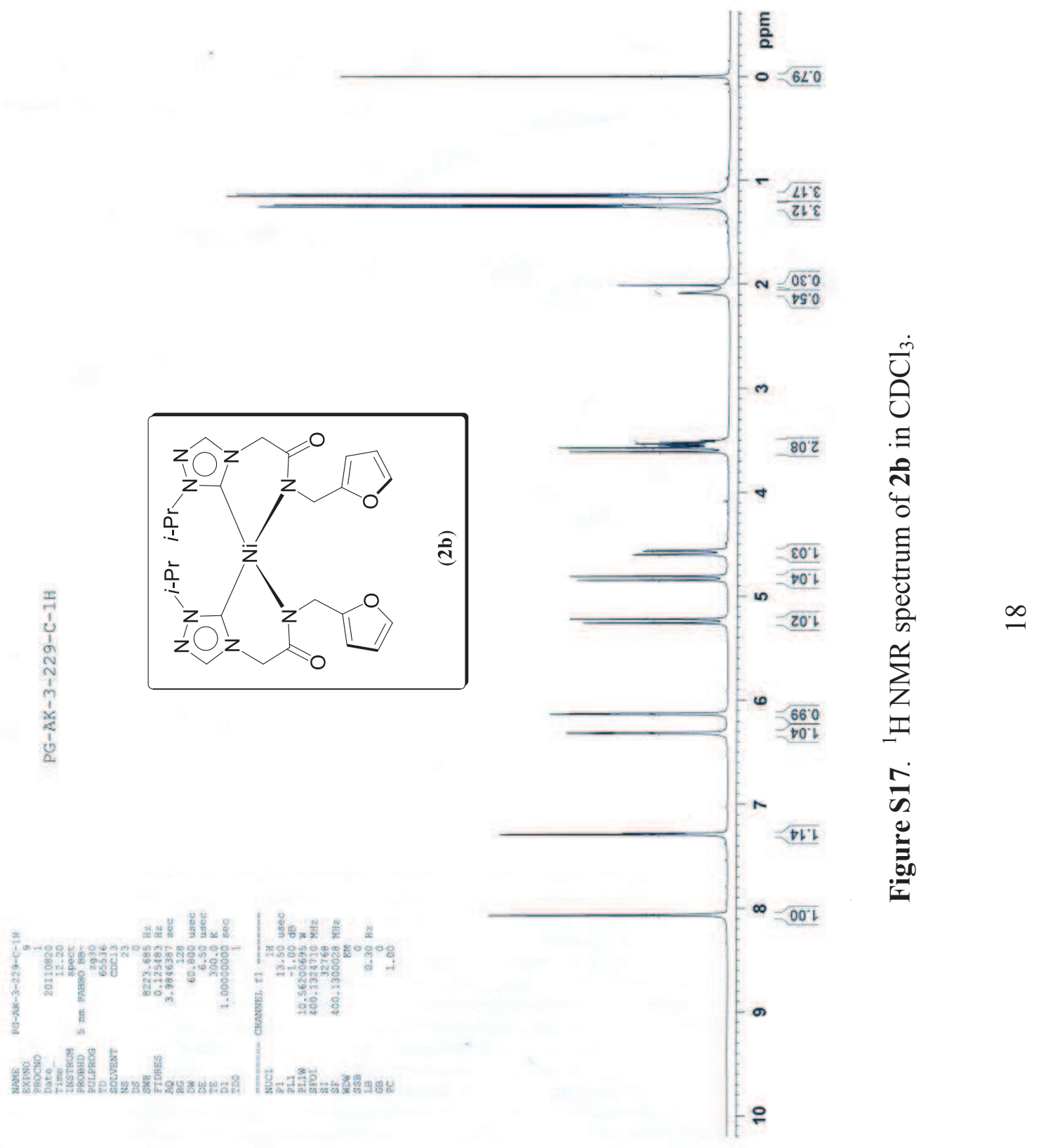




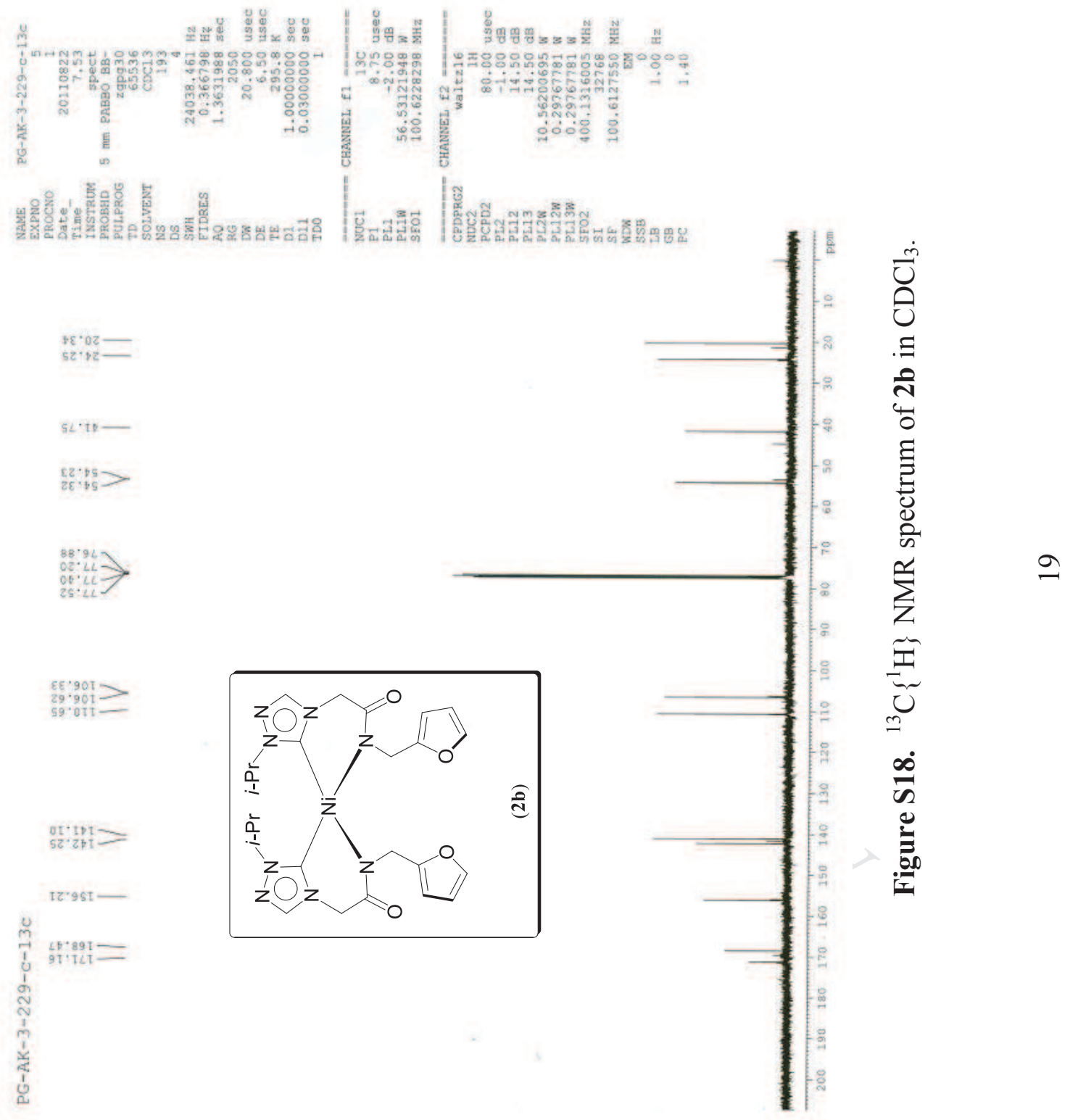




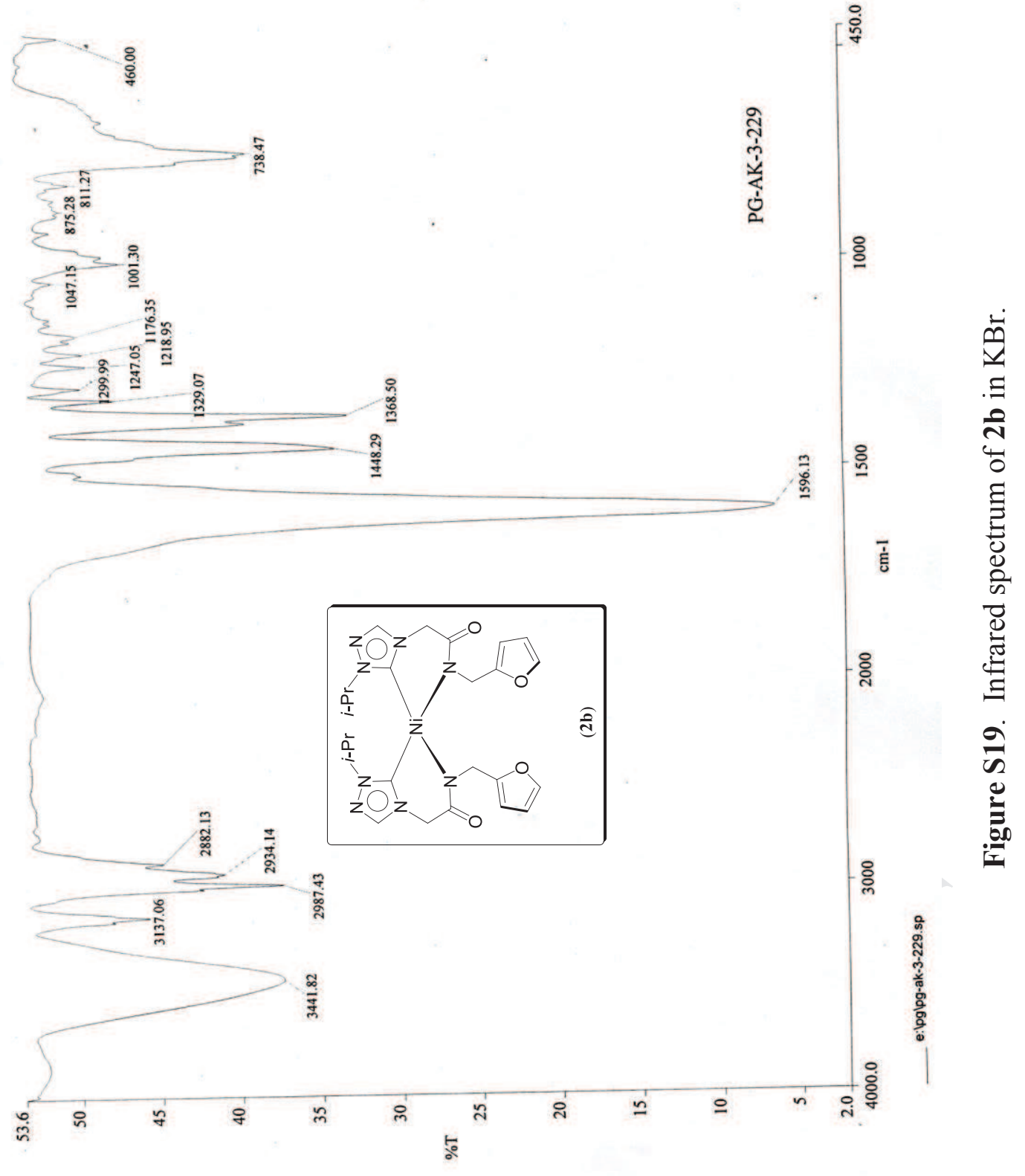




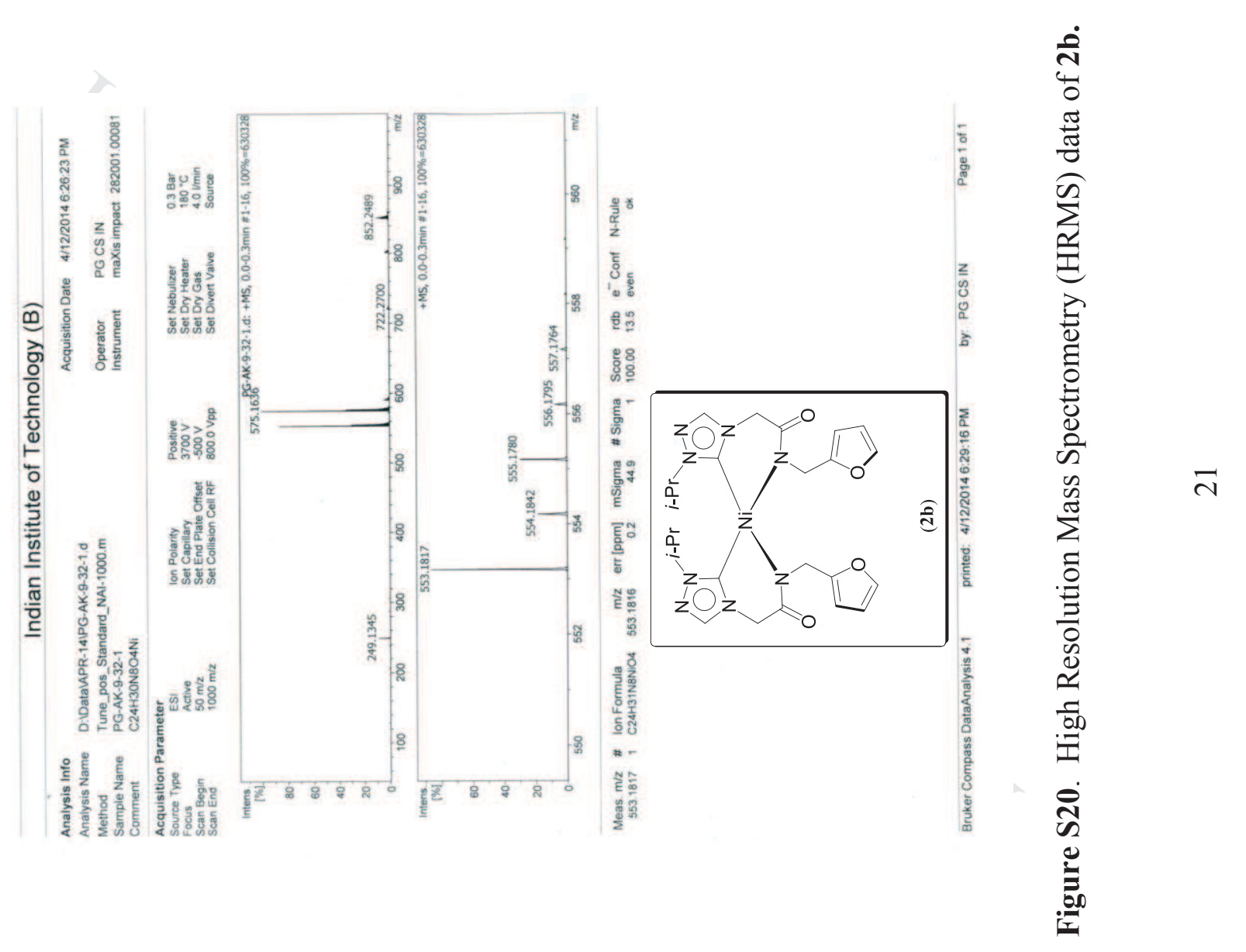



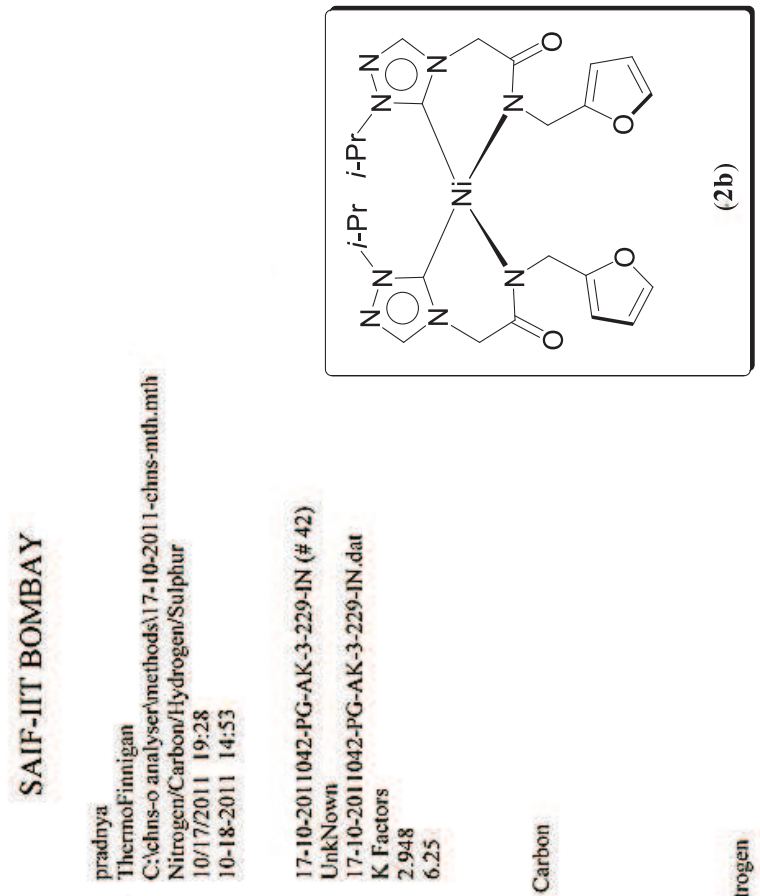

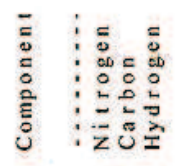
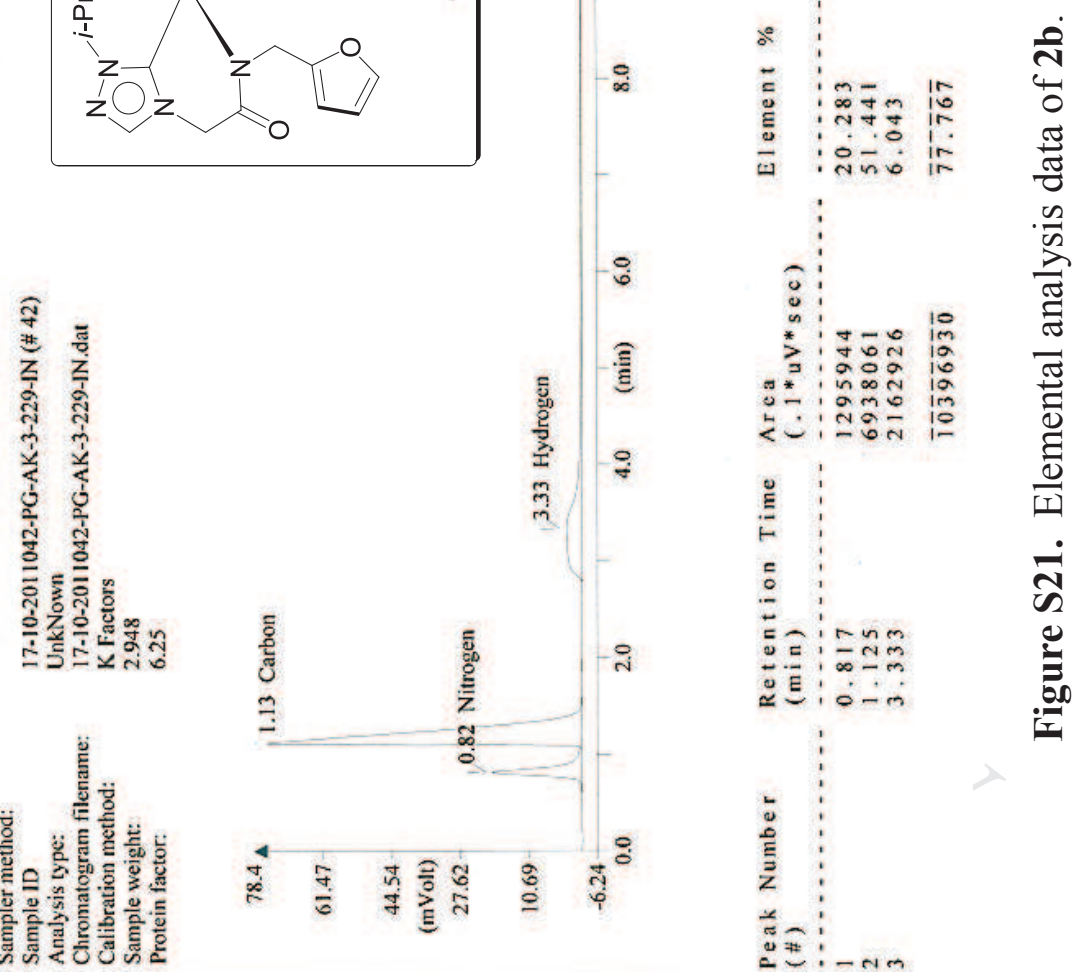


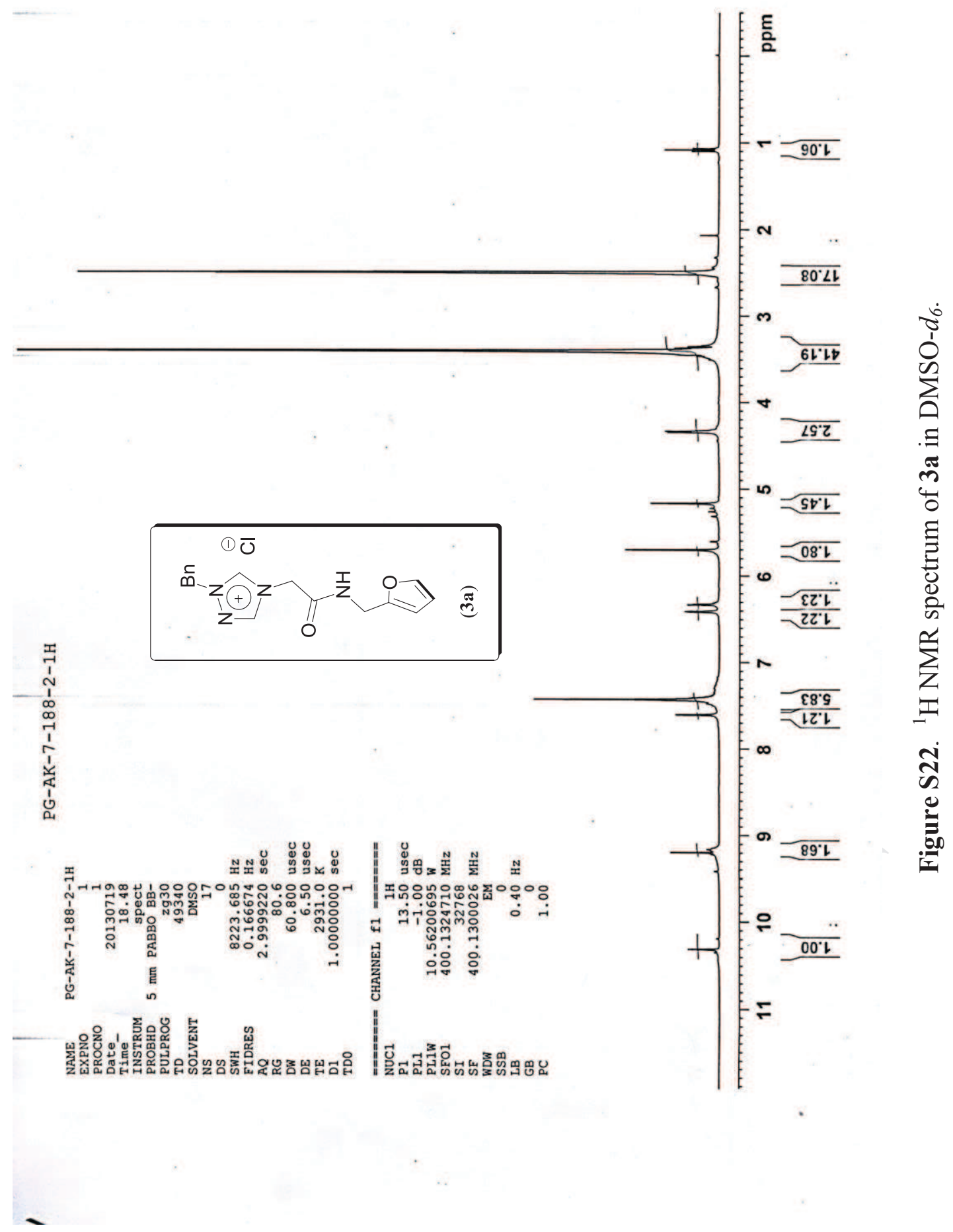




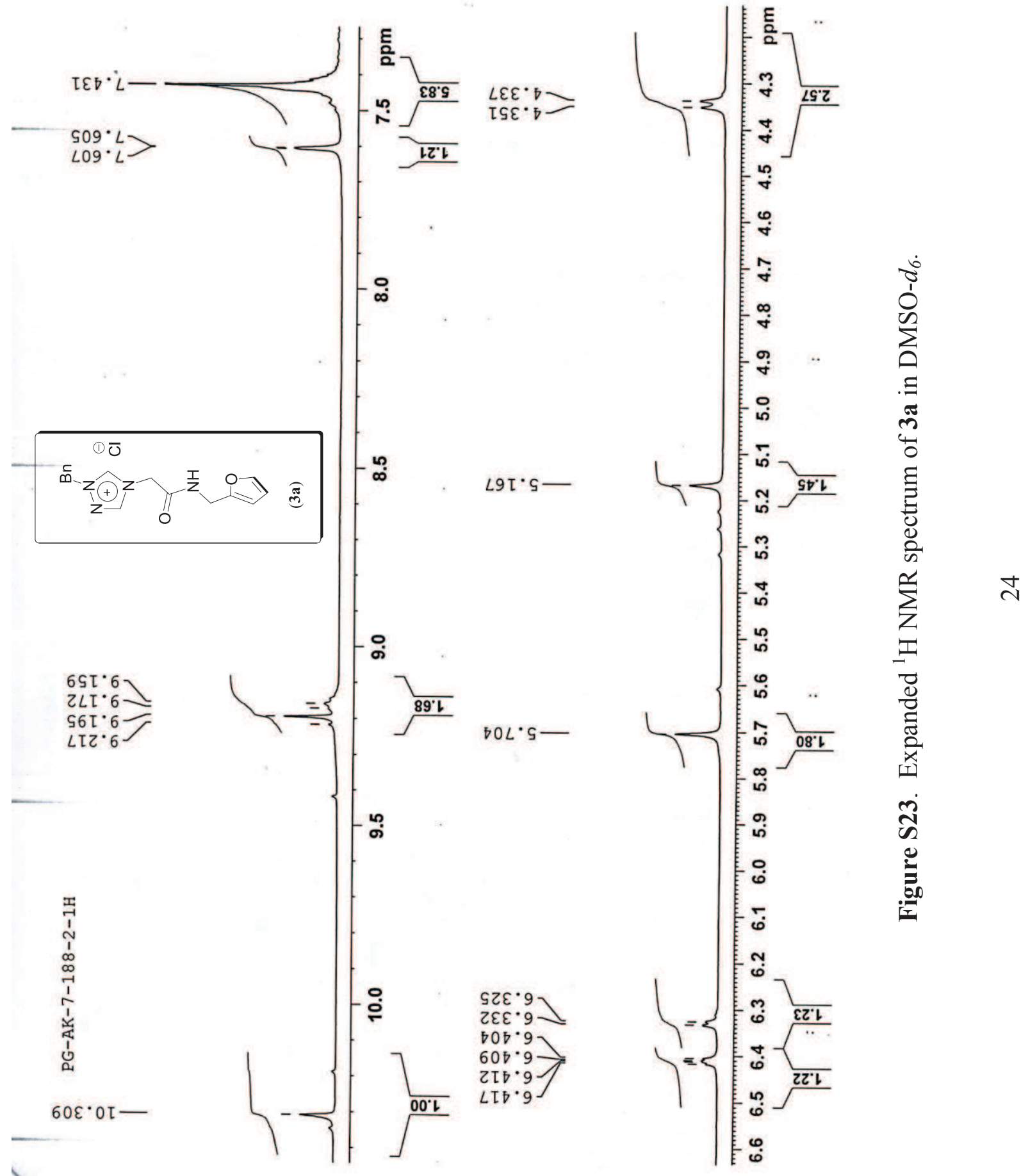




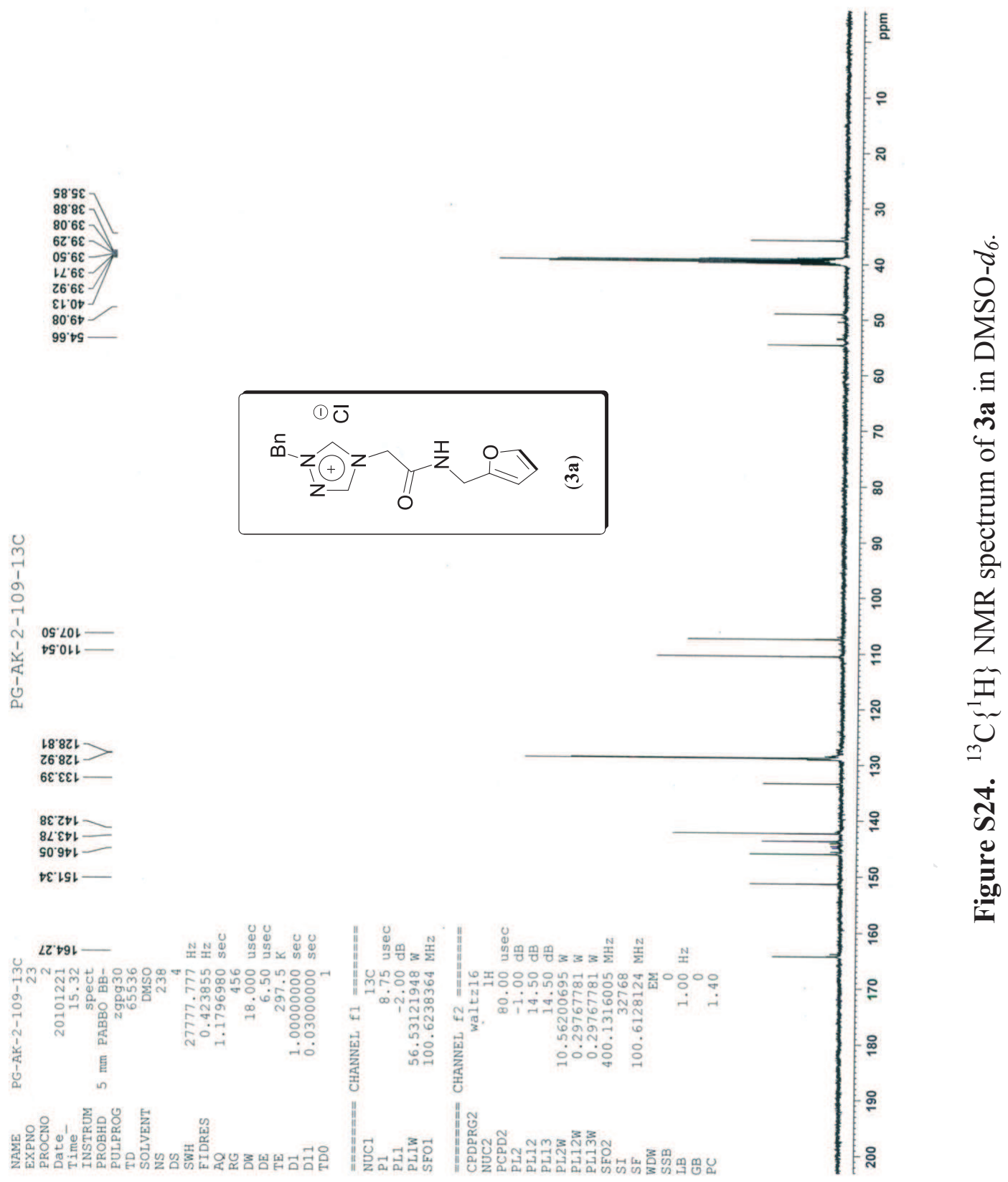




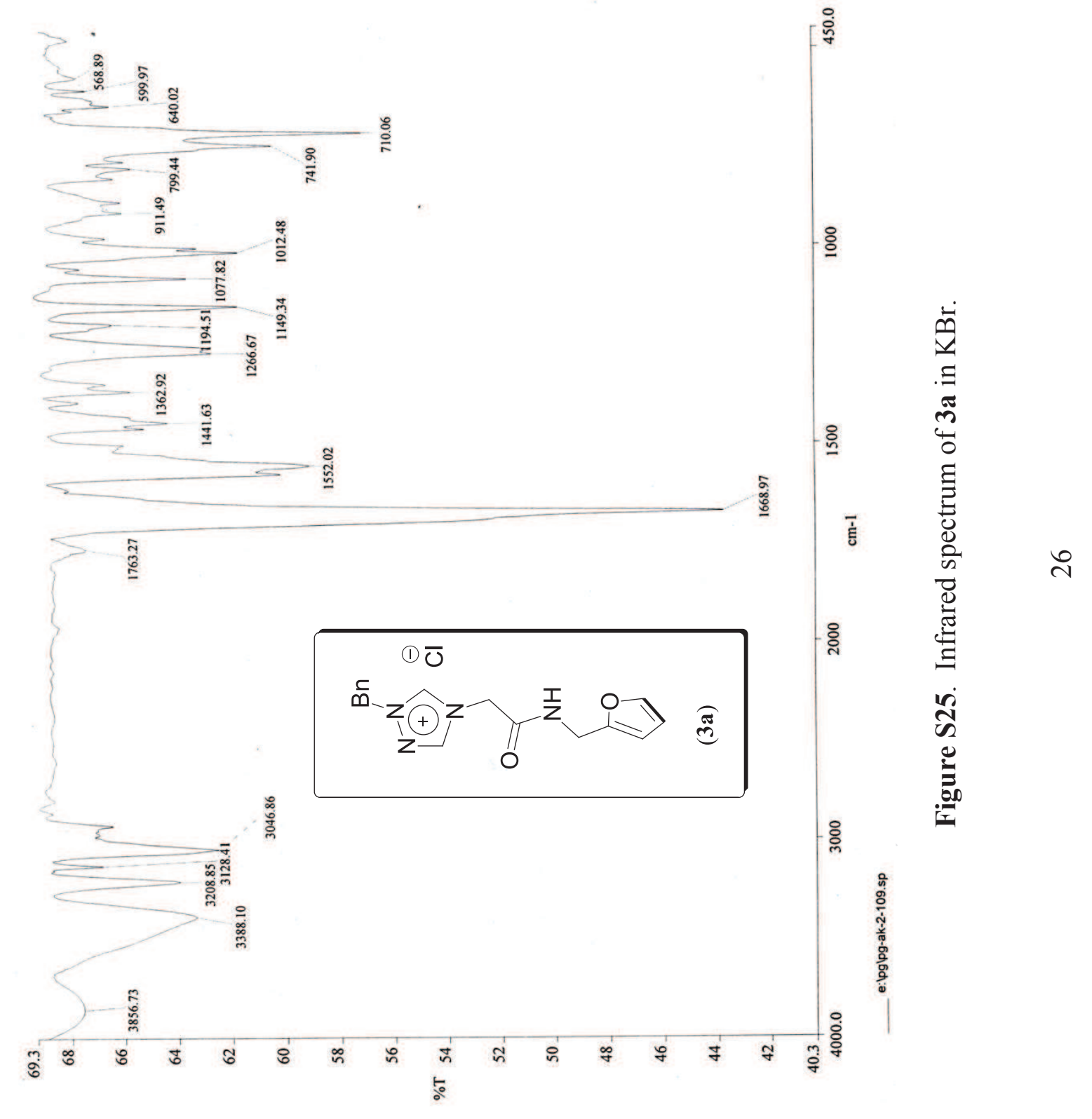




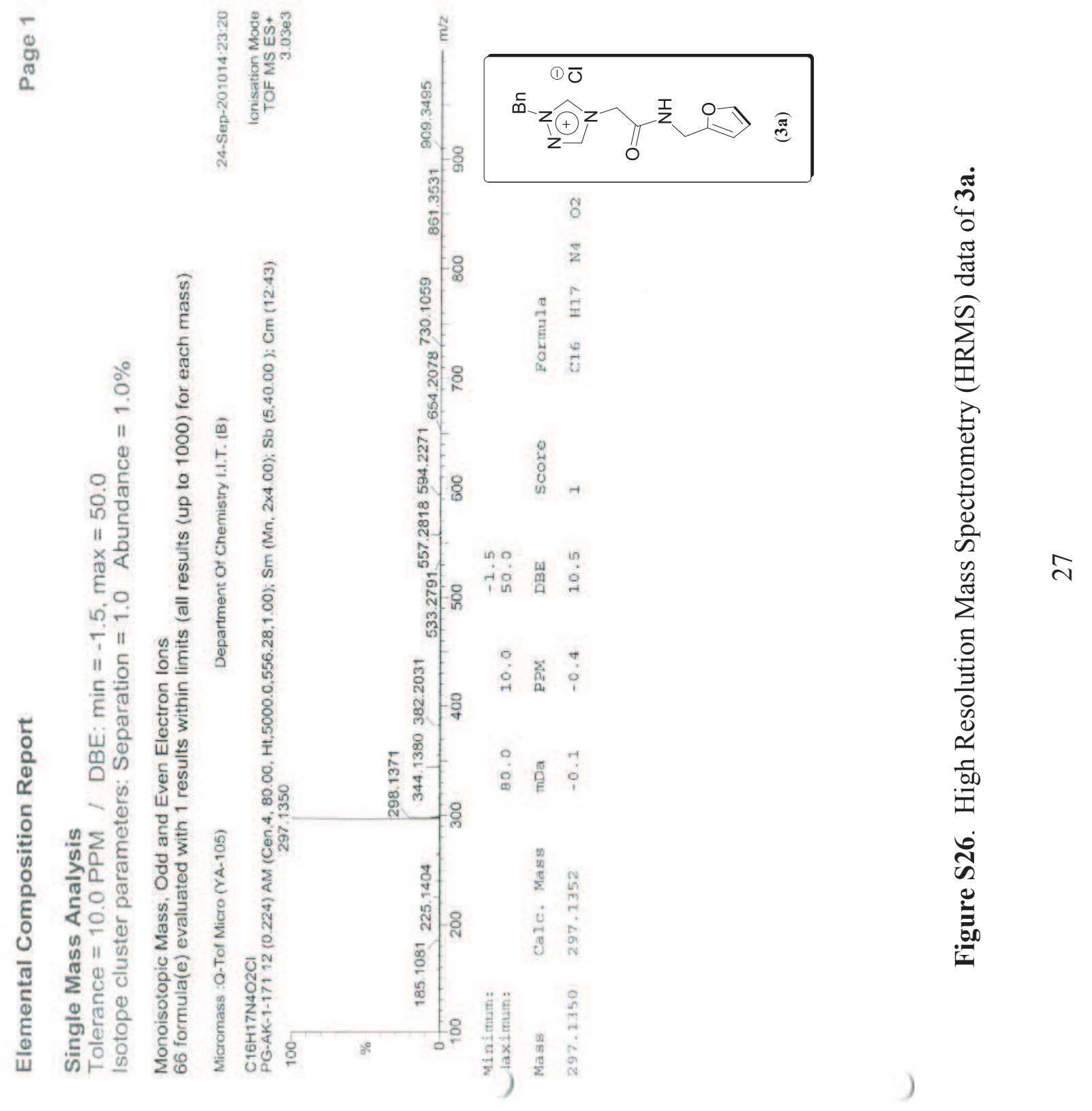




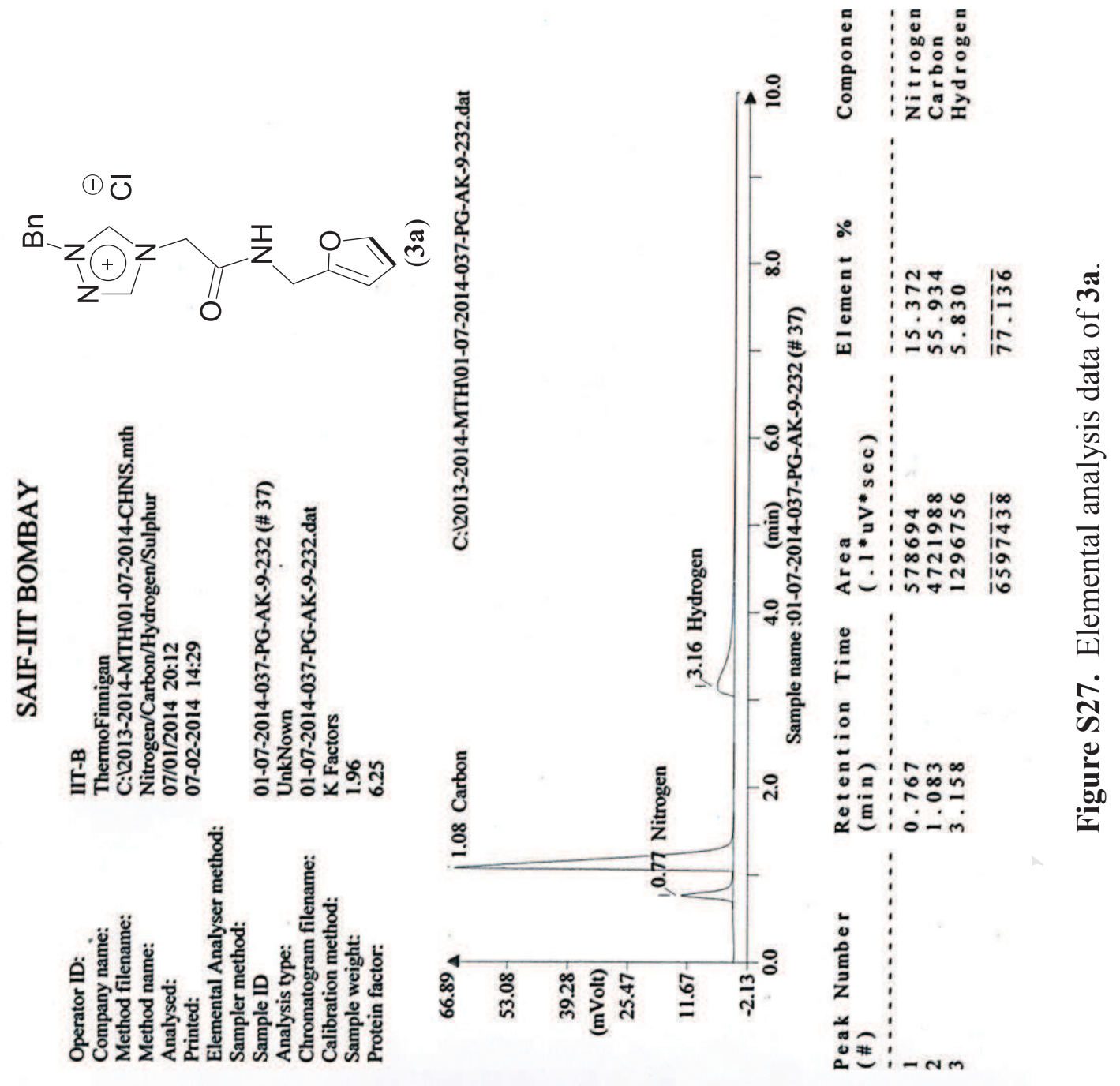




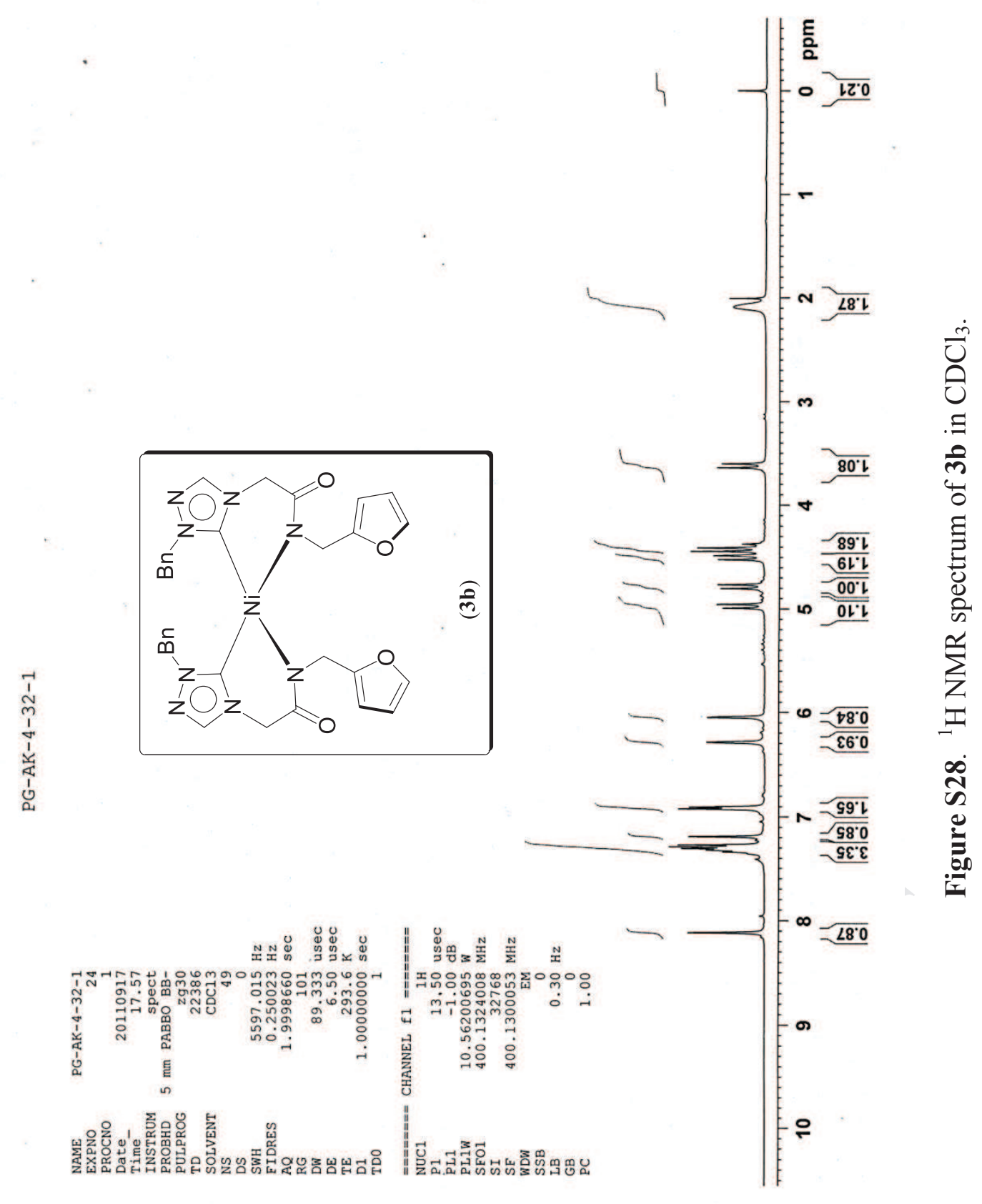




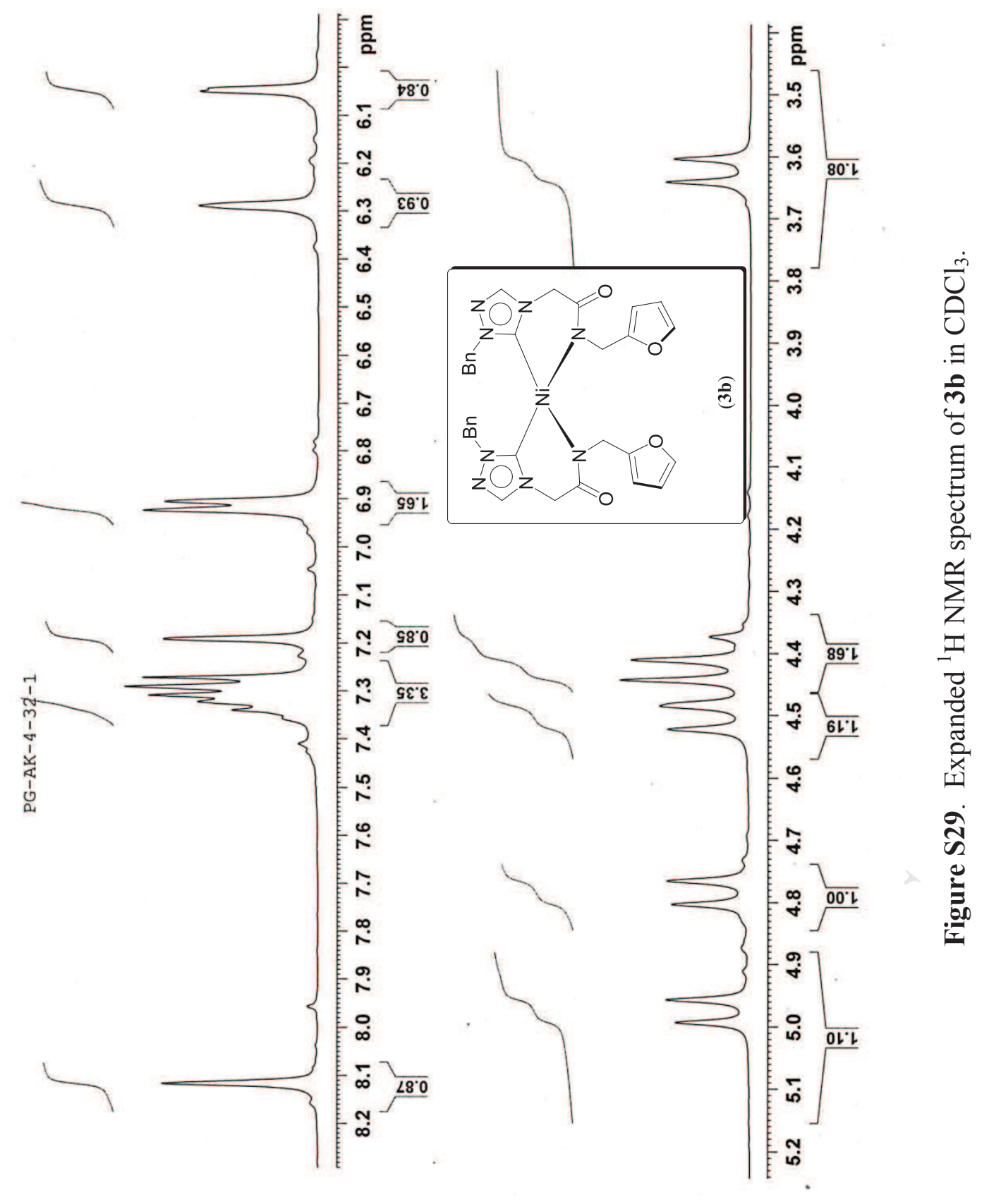




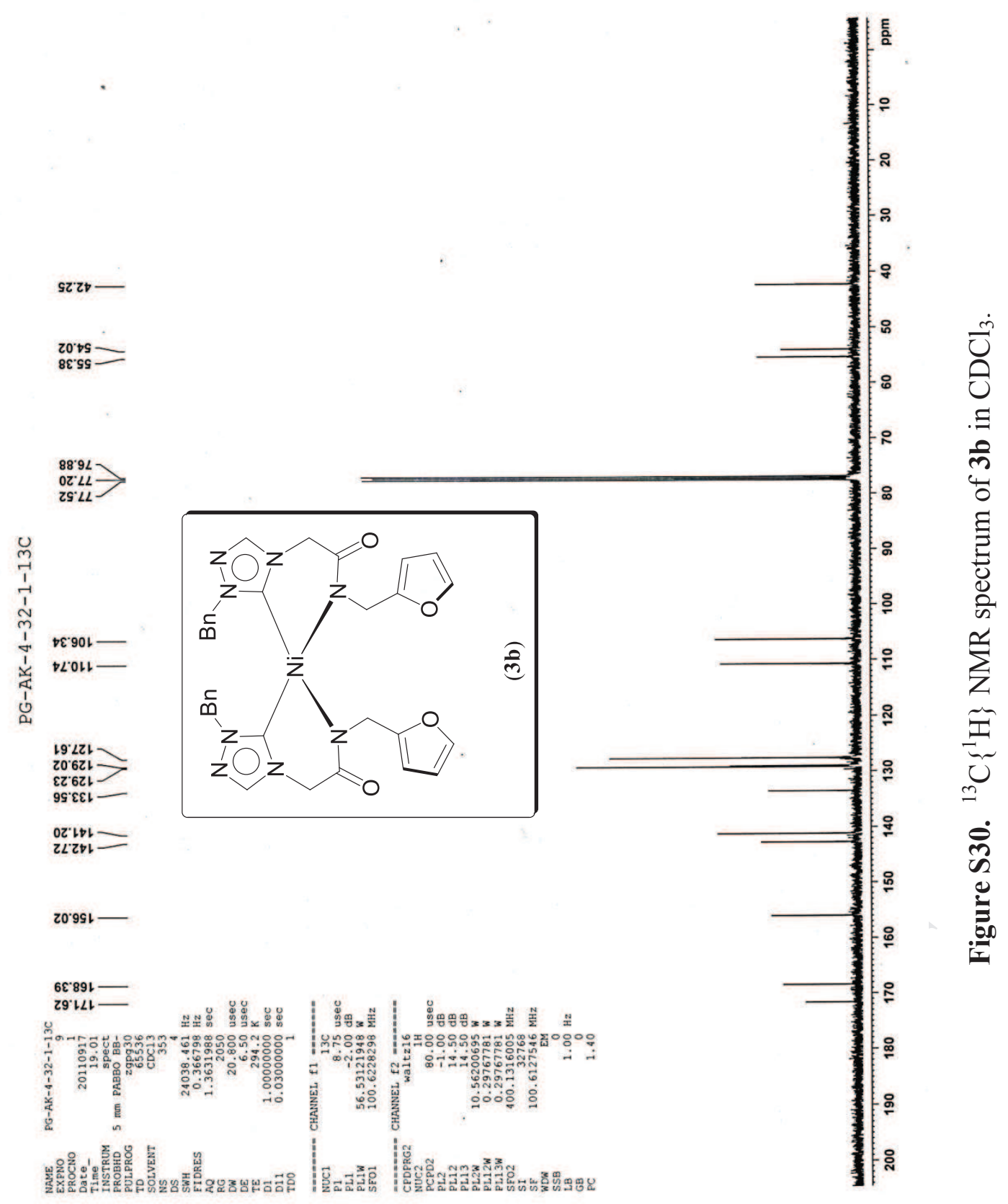




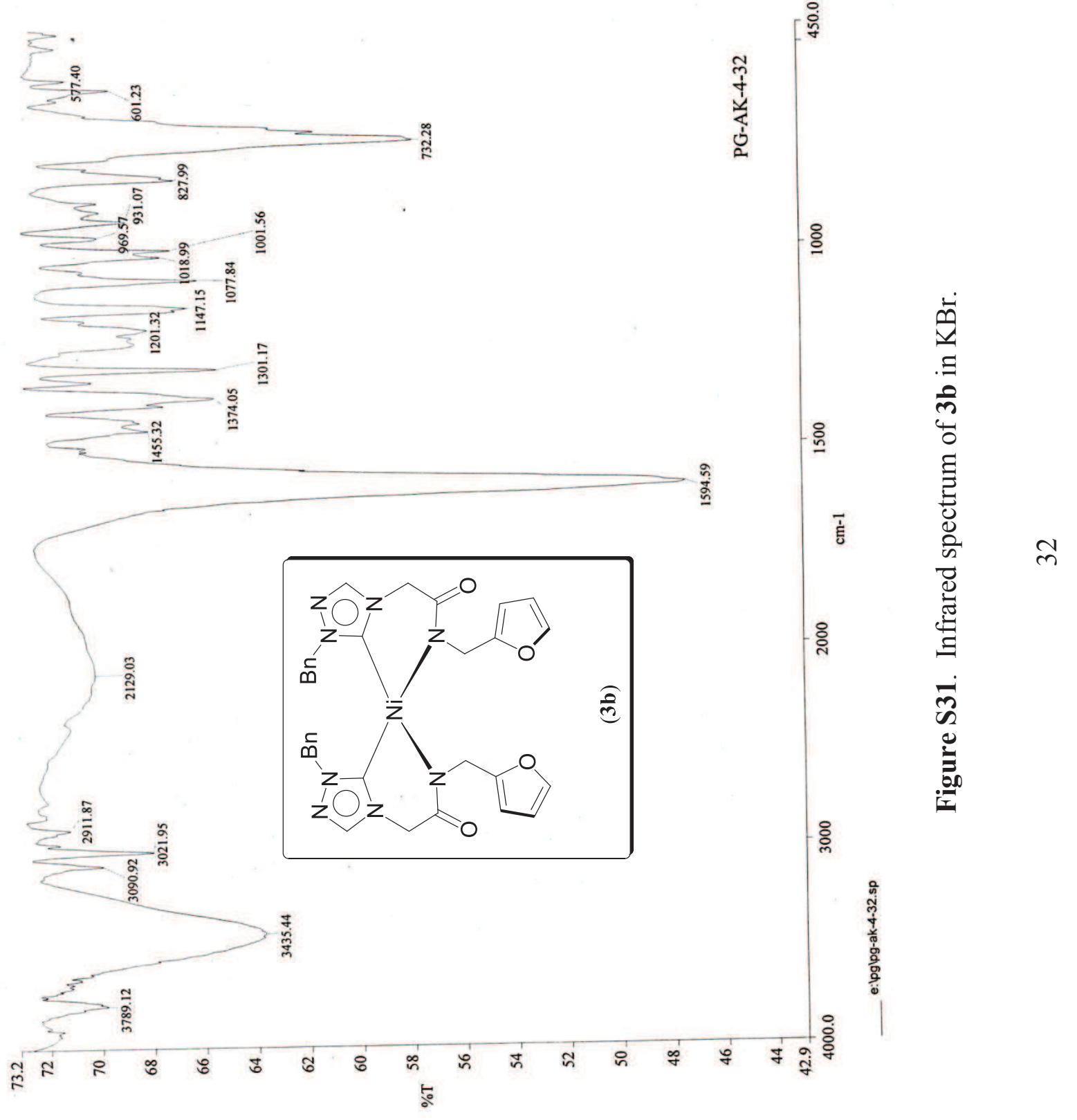




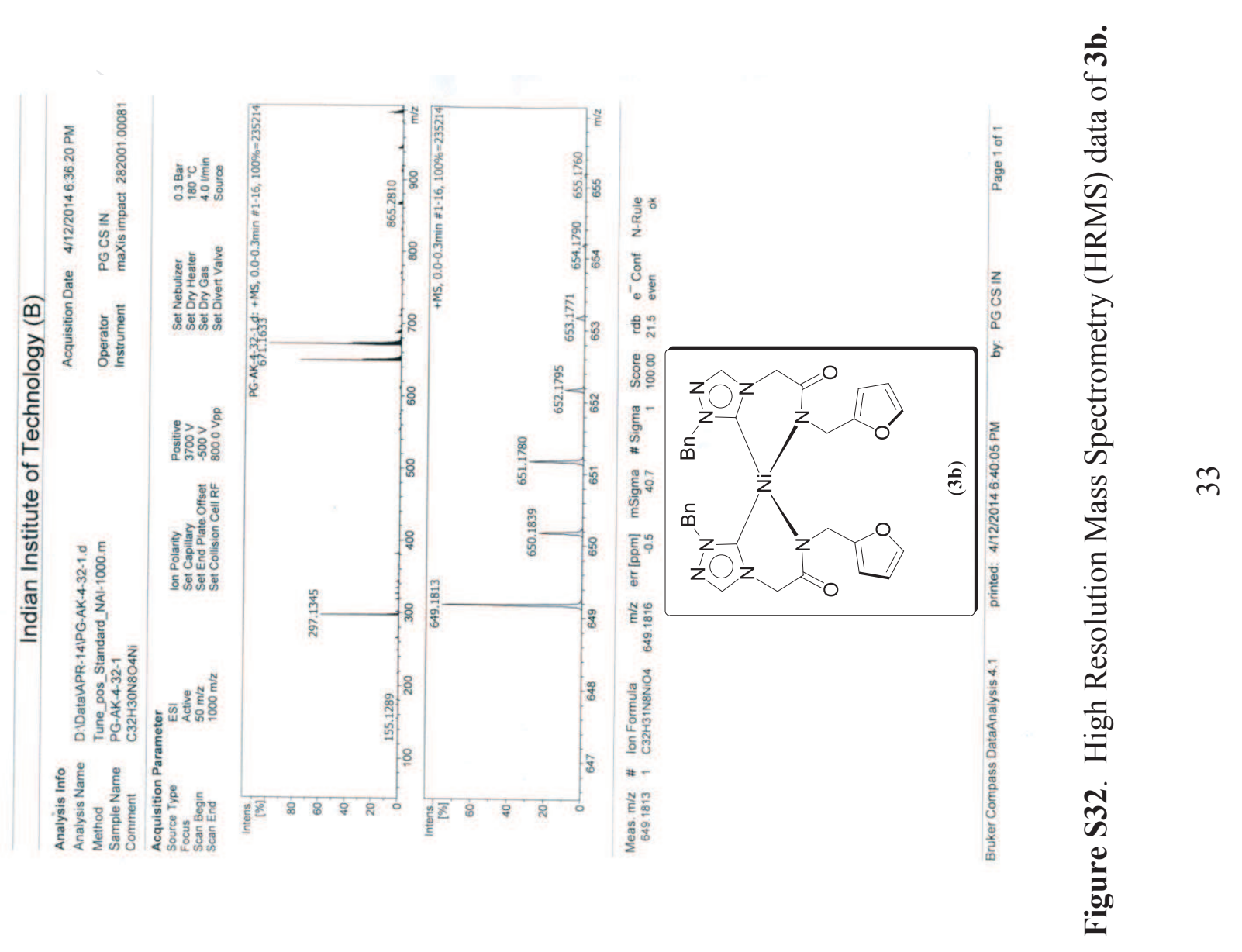




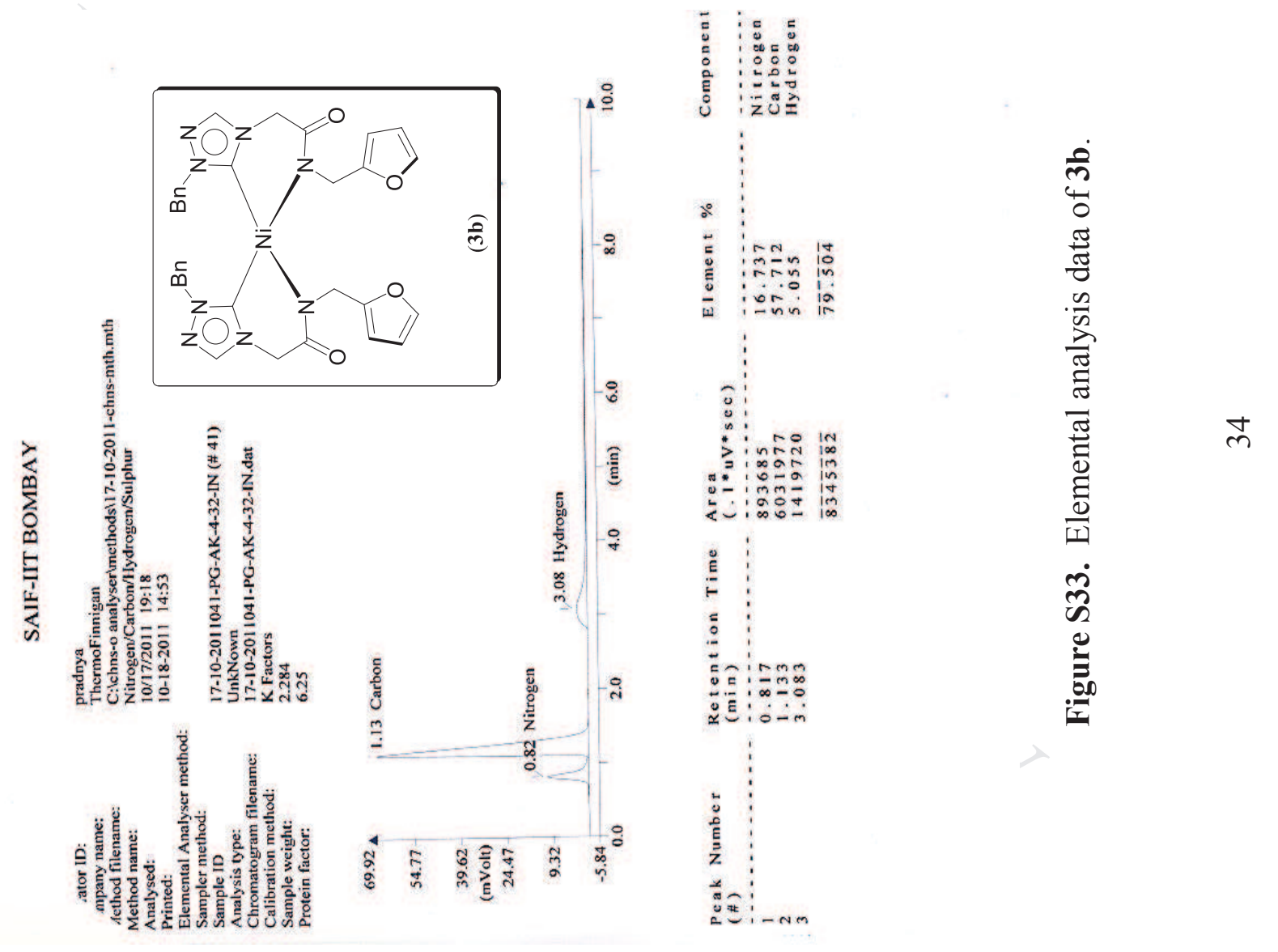




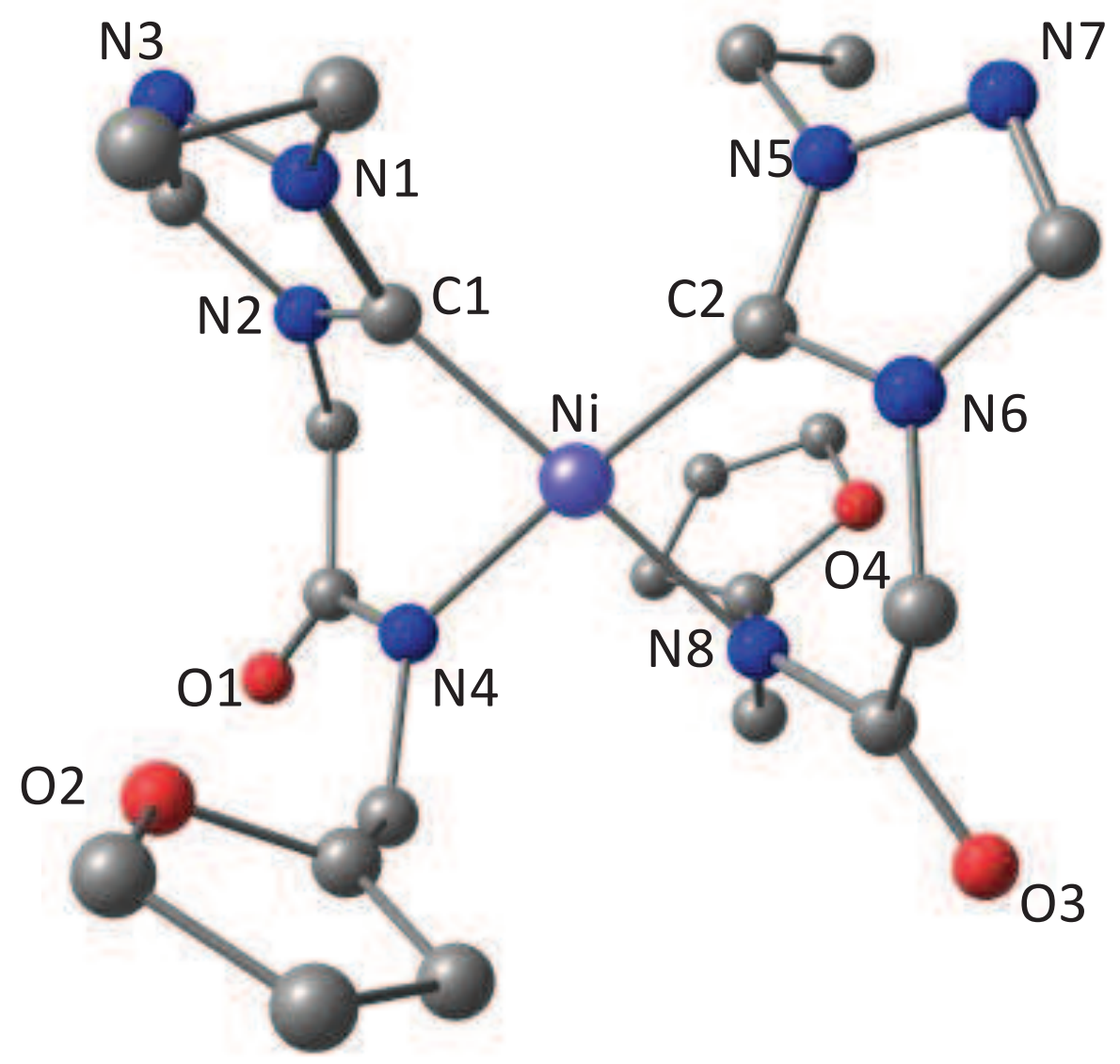

Figure S34. Computed structure of 1b. Selected bond lengths (Á), bond angles $\left(^{\circ}\right)$; $\mathrm{Ni}-\mathrm{C} 1$ 1.907, Ni-C2 1.908, Ni-N4 1.941, Ni-N8 1.941, C1-N1 1.346, C1-N2 1.364, C2-N5 1.346, C2-N6 1.364, C1-Ni-C2 97.1, N4-Ni-N8 92.3, C1-Ni-N8 175.8, C2-Ni-N4 175.6. 


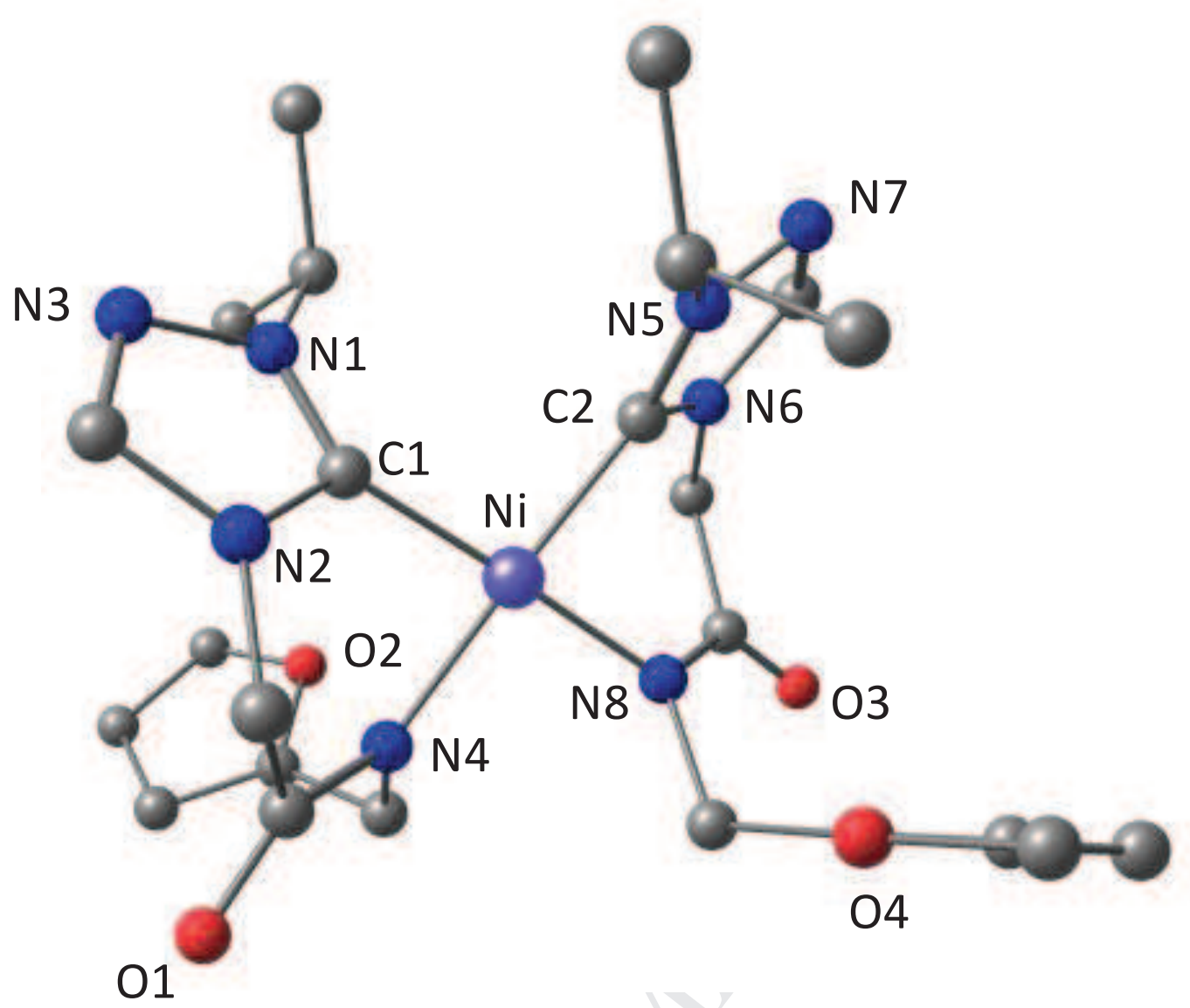

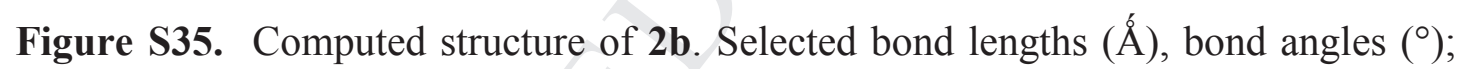
$\mathrm{Ni}-\mathrm{C} 1$ 1.908, Ni-C2 1.909, Ni-N4 1.942, Ni-N8 1.942, C1-N1 1.346, C1-N2 1.364, C2-N5 1.347, C2-N6 1.364, C1-Ni-C2 96.3, N4-Ni-N8 92.2, C1-Ni-N8 177.9, C2-Ni-N4 177.9. 


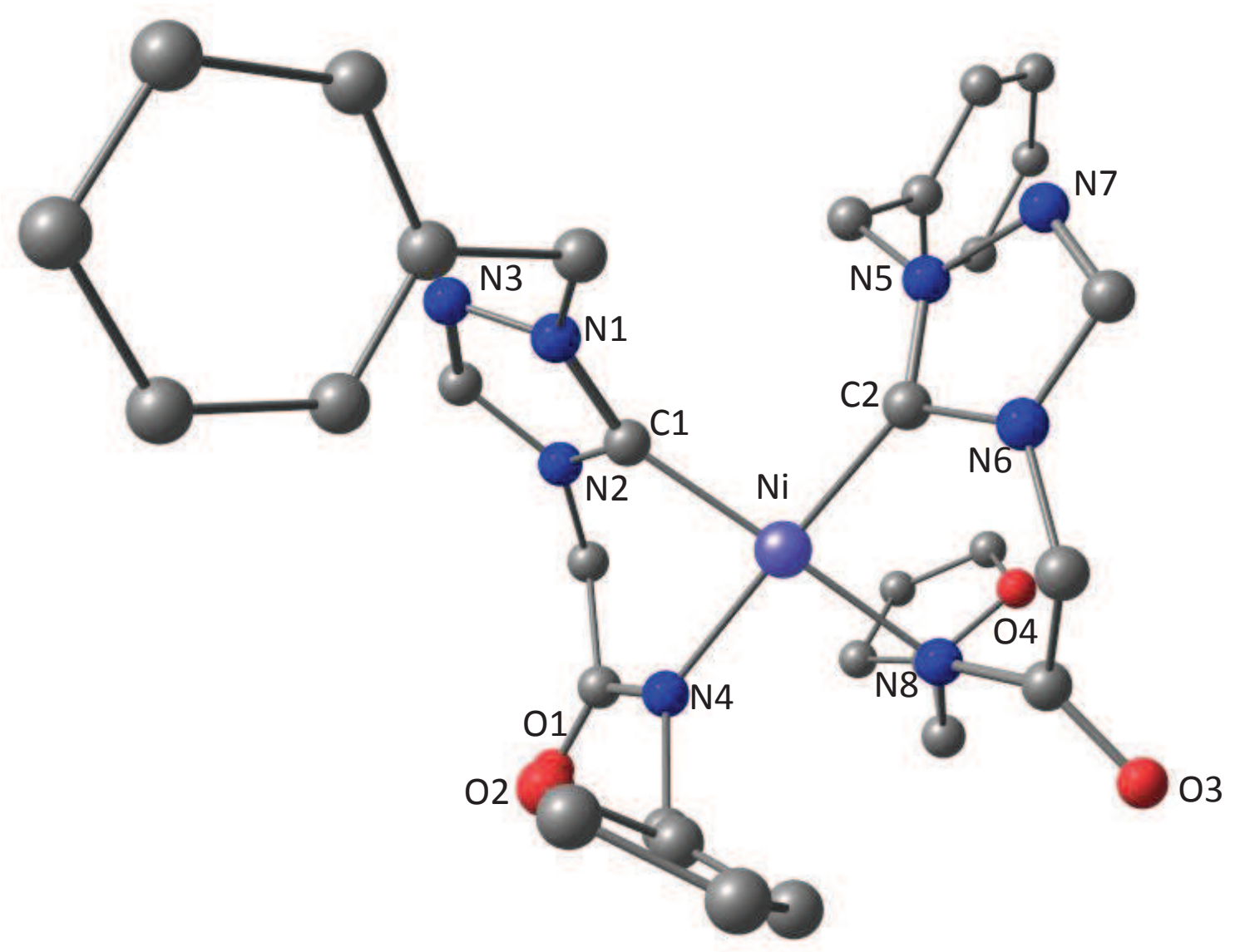

Figure S36. Computed structure of 3b. Selected bond lengths (Á), bond angles $\left(^{\circ}\right)$; $\mathrm{Ni}-\mathrm{C} 1$ 1.910, Ni-C2 1.911, Ni-N4 1.943, Ni-N8 1.941, C1-N1 1.347, C1-N2 1.363, C2-N5 1.348, C2-N6 1.363, C1-Ni-C2 96.6, N4-Ni-N8 92.5, C1-Ni-N8 174.2, $\mathrm{C} 2-\mathrm{Ni}-\mathrm{N} 4175.7$. 


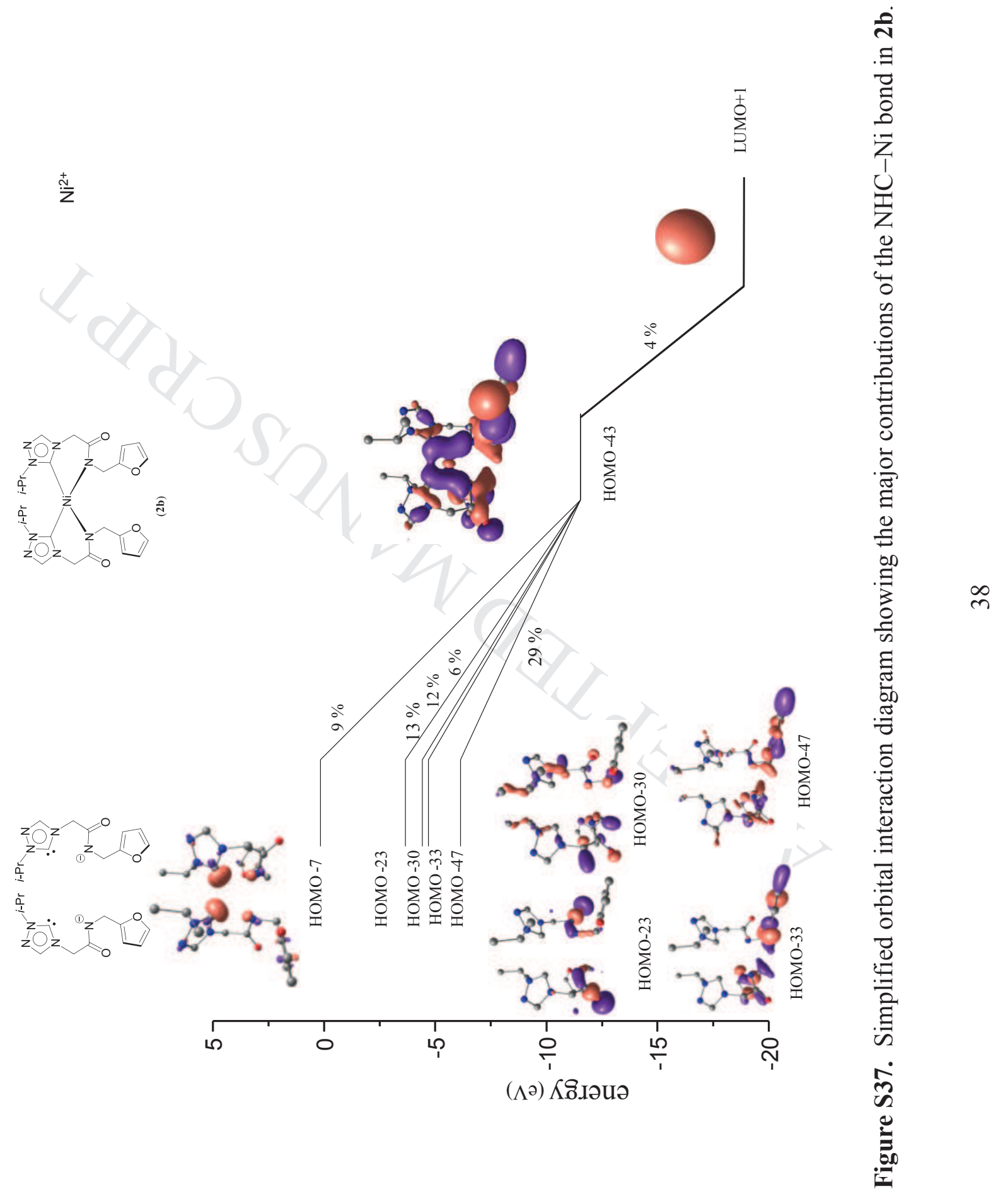




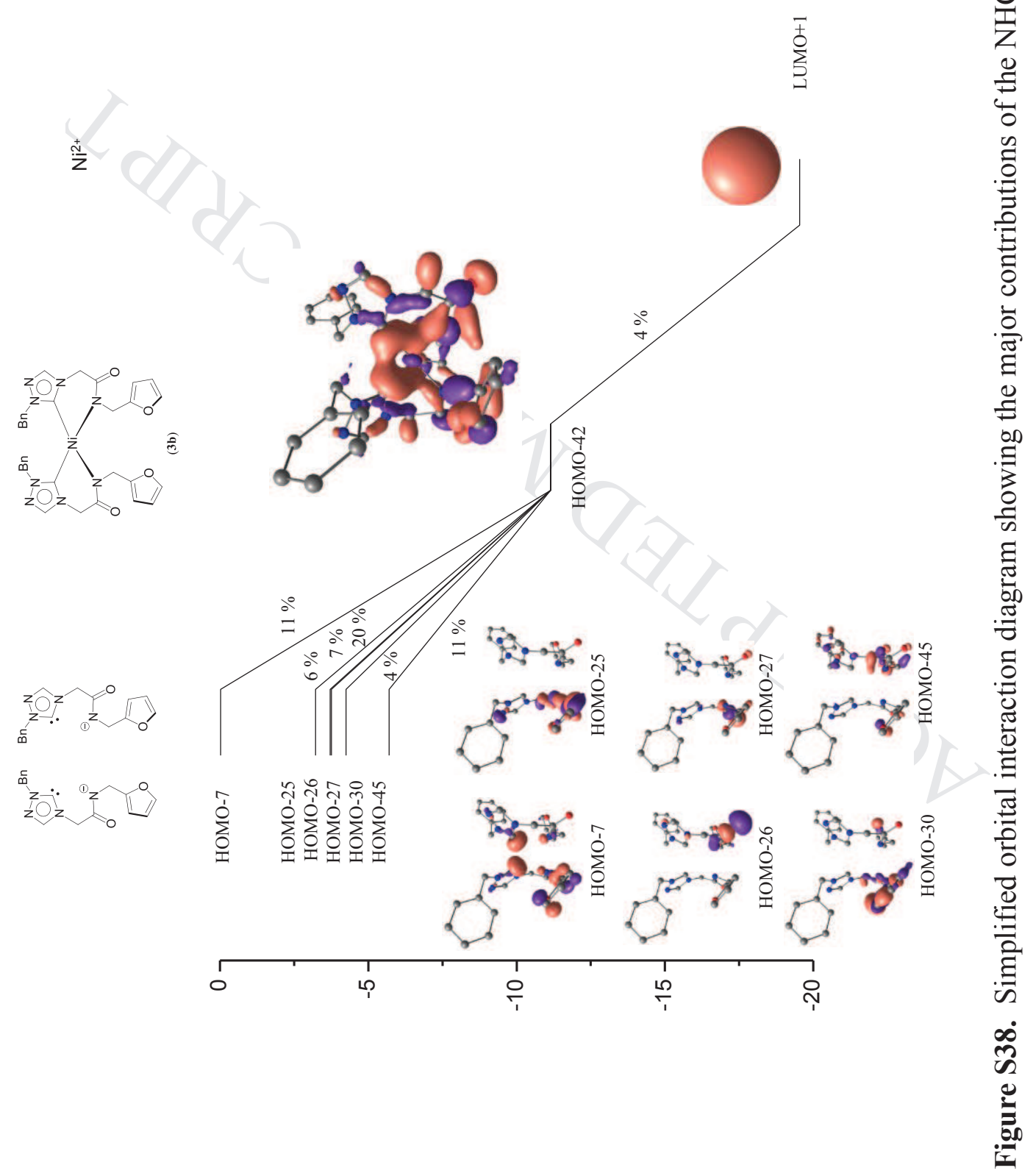


Table S1. Natural and Mulliken charge data for 1b, its NHC ligand fragment and Ni.

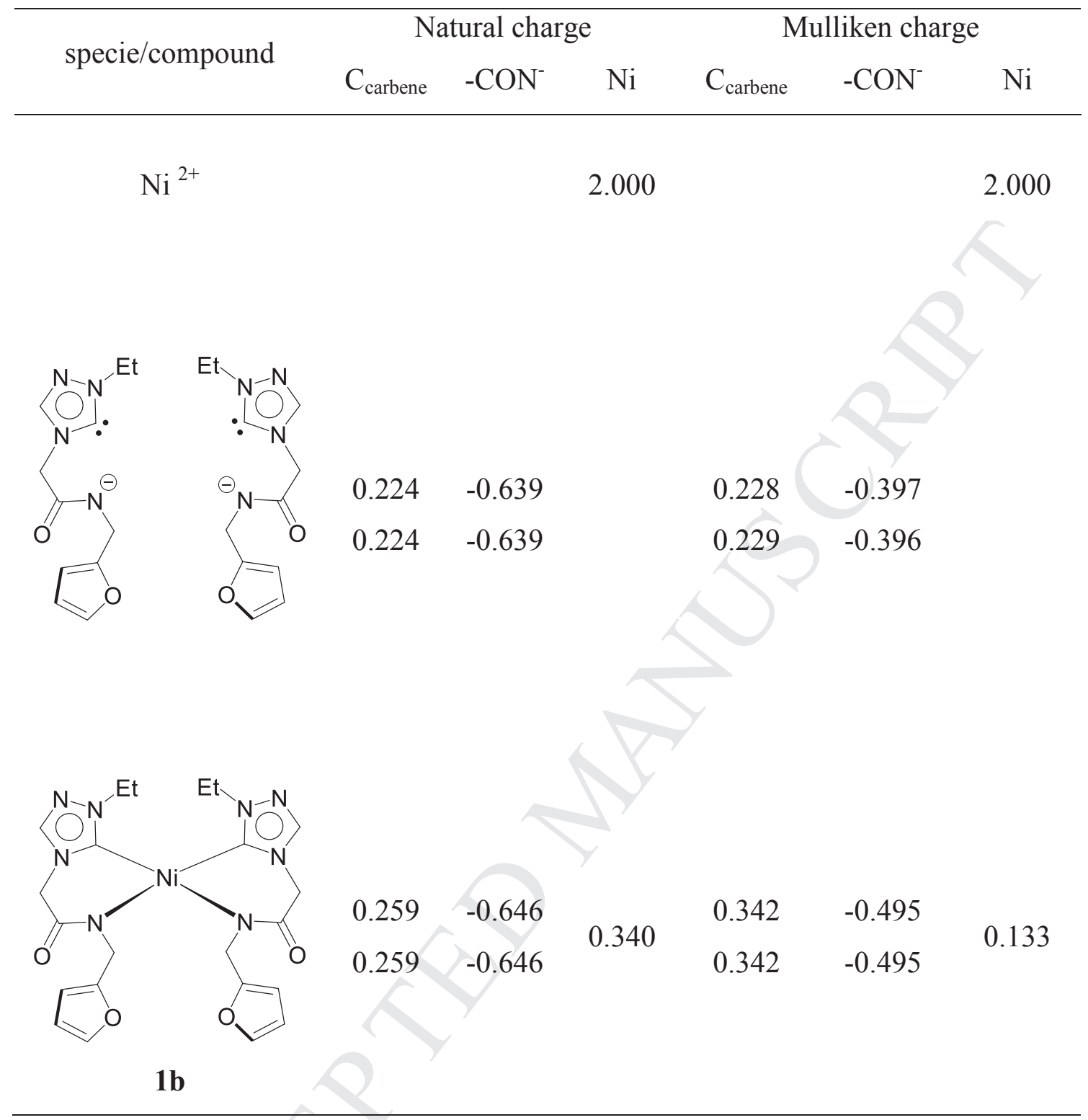


Table S2. Natural and Mulliken charge data for $\mathbf{2 b}$, its NHC ligand fragment and Ni.

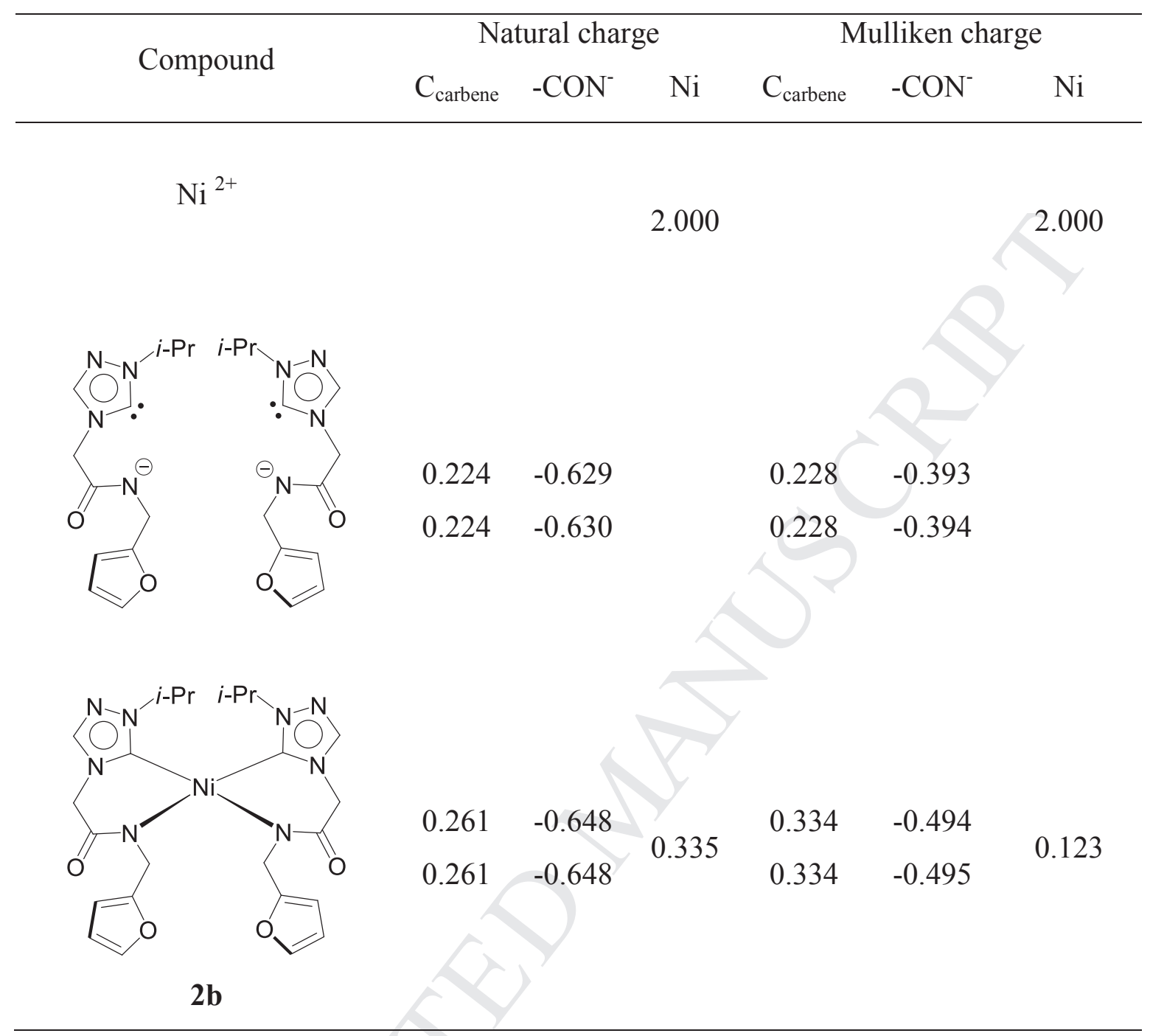


Table S3. Natural and Mulliken charge data for $\mathbf{3 b}$, its NHC ligand fragment and Ni.

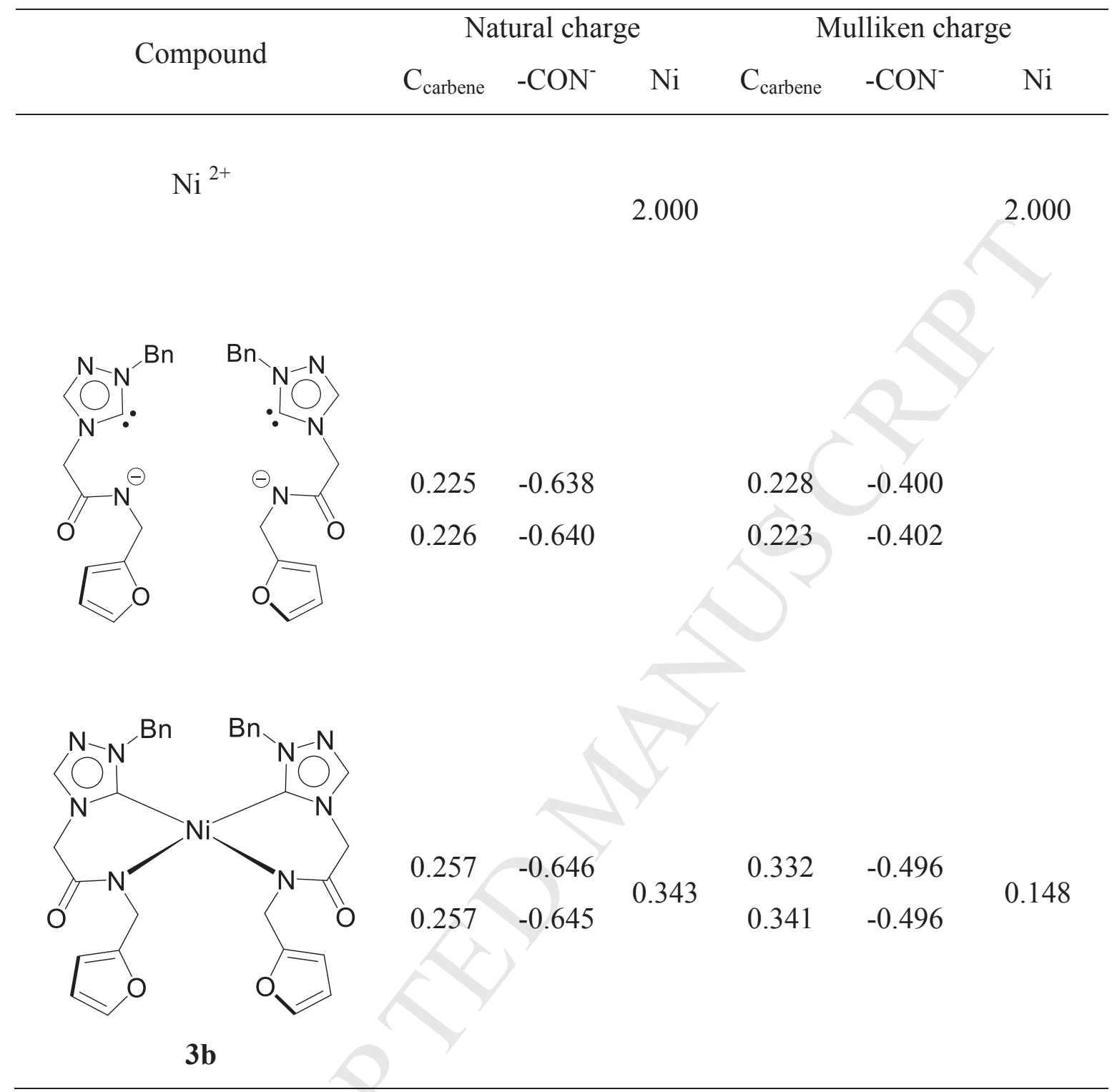


Table S4. Electronic configuration of $\mathrm{Ni}^{2+}, \mathbf{1 b}, \mathbf{2 b}$ and $\mathbf{3 b}$.

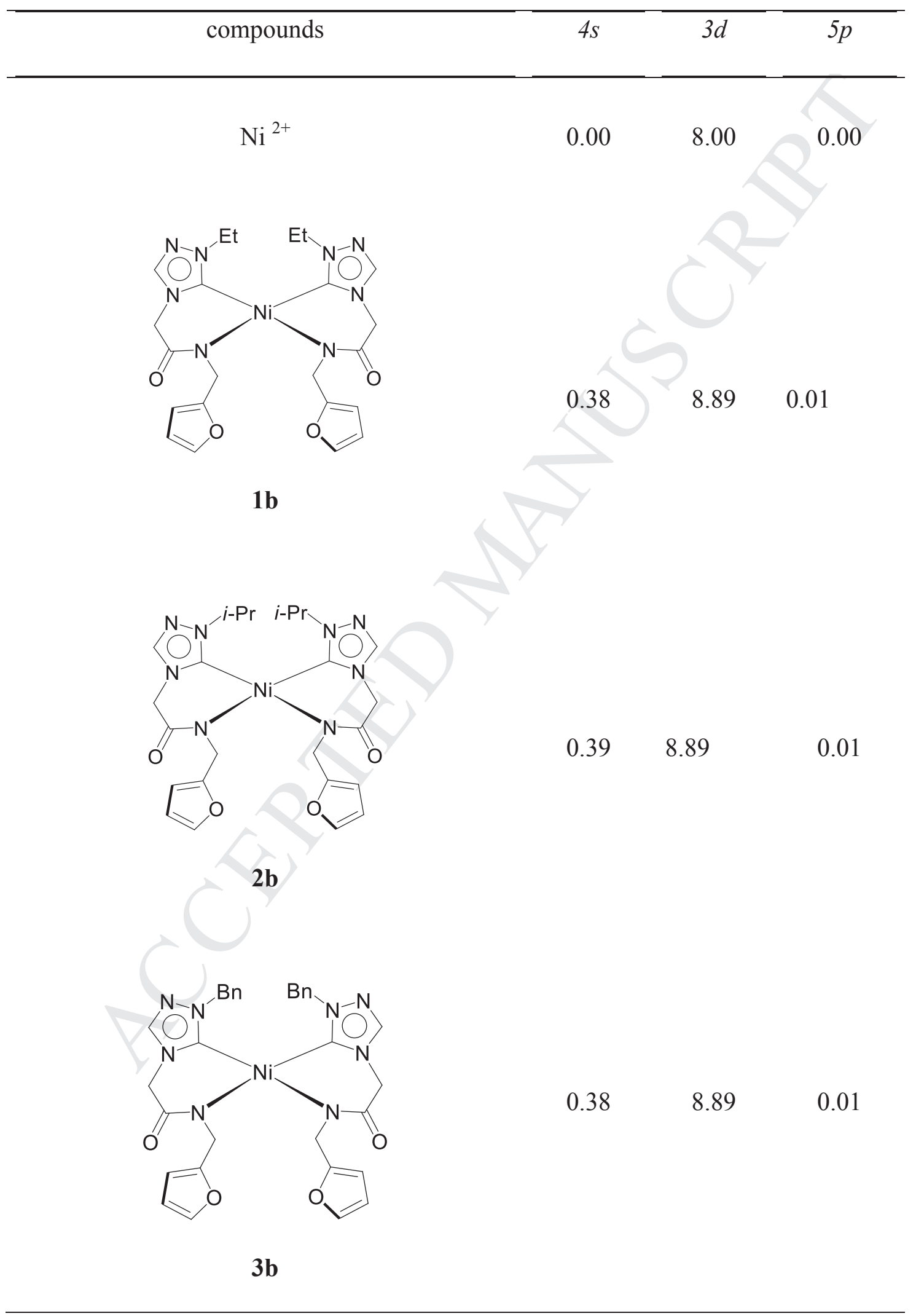


Table S5. Hybrid Orbitals of (1-3)b.

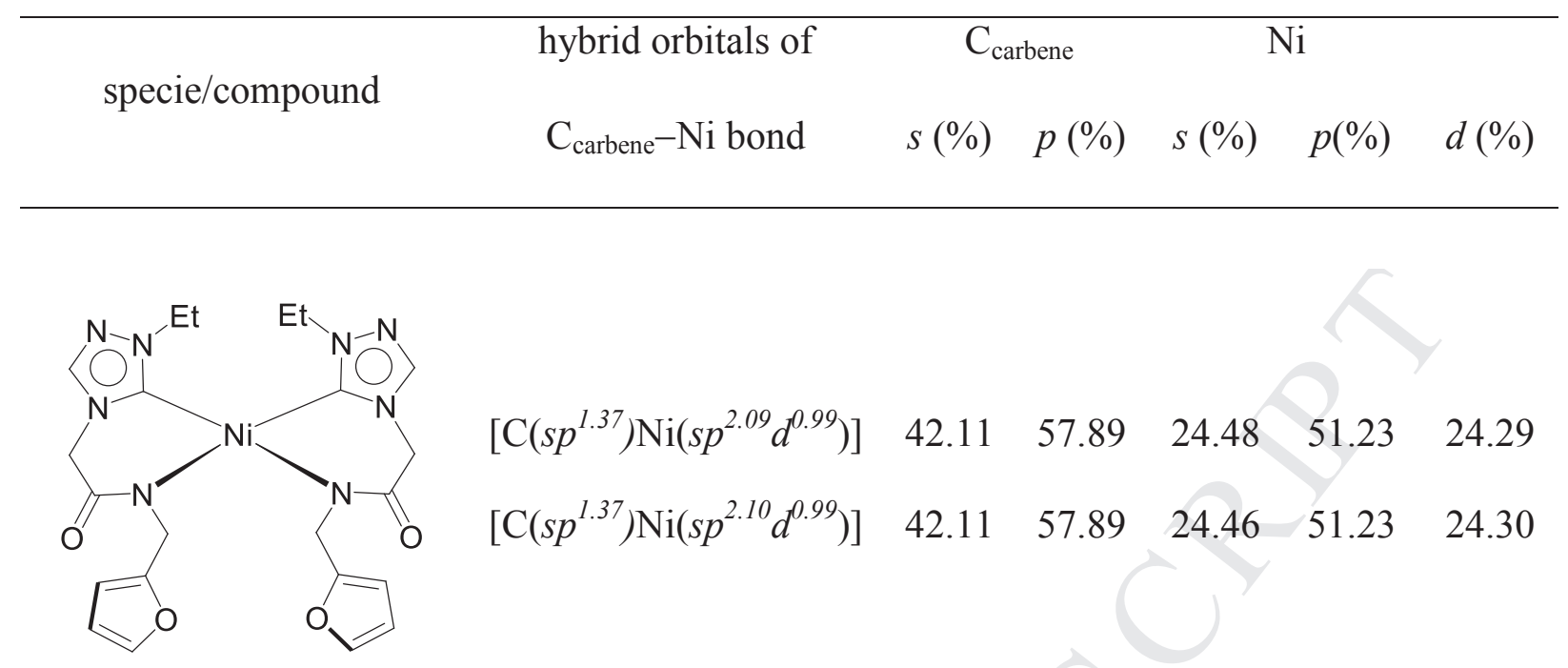

$1 b$<smiles></smiles>

2b

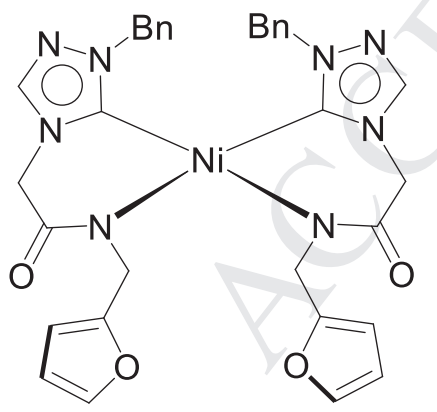

$$
\left[\mathrm{C}\left(s p^{1.37}\right) \mathrm{Ni}\left(s p^{1.90} d^{0.85}\right)\right]
$$

42.1 $\left[\mathrm{C}\left(s p^{1.38}\right) \mathrm{Ni}\left(s p^{1.92} d^{0.85}\right)\right]$ 42.02
$58.18 \quad 24.38 \quad 51.17$

24.44 $\left[\mathrm{C}\left(s p^{1.39}\right) \mathrm{Ni}\left(s p^{2.10} d^{1.00}\right)\right] \quad 41.81 \quad 58.18 \quad 24.38 \quad 51.15 \quad 24.47$

$3 b$ 


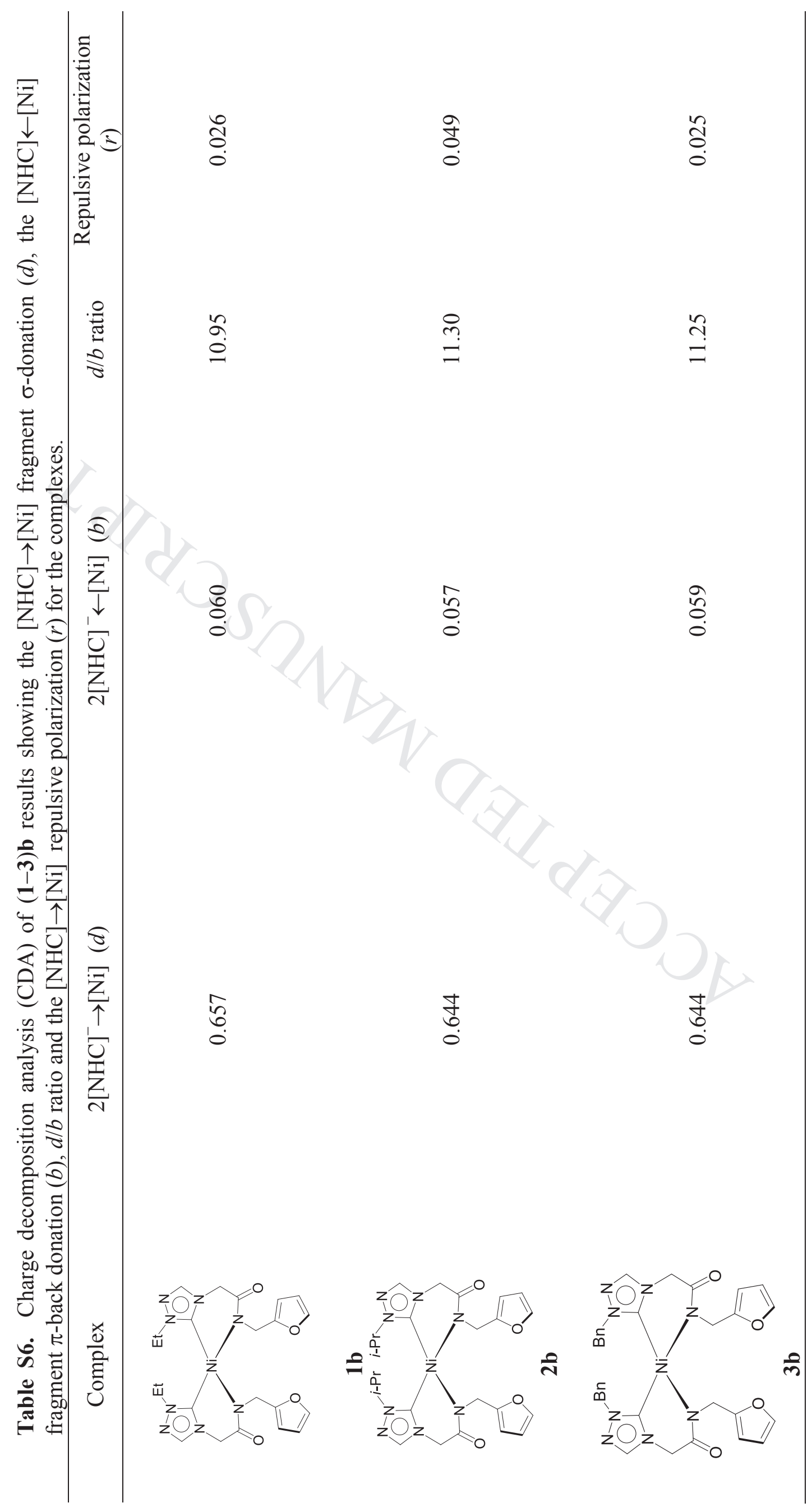


Table S7. B3LYP/6-31G*, LANL2DZ level optimized coordinates of $\mathbf{1 b .}$

Ground state electronic energy $=-1762.1629142$ Hartree/Particle.

\begin{tabular}{|c|c|c|c|}
\hline $\mathrm{Ni}$ & 6.615628000 & 8.324206000 & 3.135501000 \\
\hline $\mathrm{O}$ & 5.610616000 & 4.889824000 & 5.114982000 \\
\hline $\mathrm{O}$ & 9.468045000 & 6.888036000 & 5.381643000 \\
\hline $\mathrm{O}$ & 8.581309000 & 7.276072000 & -0.290246000 \\
\hline $\mathrm{O}$ & 4.212487000 & 7.484412000 & 0.225537000 \\
\hline $\mathrm{N}$ & 6.446842000 & 10.039648000 & 5.643320000 \\
\hline $\mathrm{N}$ & 5.787852000 & 10.033404000 & 6.851905000 \\
\hline $\mathrm{N}$ & 5.170397000 & 8.344542000 & 5.570067000 \\
\hline $\mathrm{N}$ & 6.738727000 & 6.611913000 & 4.041305000 \\
\hline $\mathrm{N}$ & 5.842891000 & 11.069519000 & 2.070132000 \\
\hline $\mathrm{N}$ & 6.314119000 & 11.989456000 & 1.160757000 \\
\hline $\mathrm{N}$ & 7.658860000 & 10.247028000 & 1.339675000 \\
\hline $\mathrm{N}$ & 7.024254000 & 7.529600000 & 1.412721000 \\
\hline $\mathrm{C}$ & 6.103945000 & 9.016931000 & 4.837542000 \\
\hline $\mathrm{C}$ & 5.019848000 & 8.981672000 & 6.772098000 \\
\hline $\mathrm{H}$ & 4.345282000 & 8.652686000 & 7.548973000 \\
\hline $\mathrm{C}$ & 7.474901000 & 11.058785000 & 5.425471000 \\
\hline $\mathrm{H}$ & 7.012417000 & 12.032319000 & 5.615924000 \\
\hline $\mathrm{H}$ & 7.746161000 & 11.002471000 & 4.370756000 \\
\hline $\mathrm{C}$ & 8.693629000 & 10.847355000 & 6.322131000 \\
\hline $\mathrm{H}$ & 8.403783000 & 10.866780000 & 7.376624000 \\
\hline $\mathrm{H}$ & 9.420199000 & 11.648226000 & 6.147256000 \\
\hline $\mathrm{H}$ & 9.173703000 & 9.888199000 & 6.104547000 \\
\hline $\mathrm{C}$ & 4.615809000 & 7.054802000 & 5.147858000 \\
\hline $\mathrm{H}$ & 3.930342000 & 7.212477000 & 4.309109000 \\
\hline $\mathrm{H}$ & 4.066990000 & 6.621673000 & 5.984495000 \\
\hline $\mathrm{C}$ & 5.738661000 & 6.066377000 & 4.750664000 \\
\hline $\mathrm{C}$ & 7.918927000 & 5.765688000 & 3.844712000 \\
\hline $\mathrm{H}$ & 7.972749000 & 5.377515000 & 2.821309000 \\
\hline $\mathrm{H}$ & 7.824926000 & 4.900043000 & 4.511609000 \\
\hline $\mathrm{C}$ & 9.206048000 & 6.479071000 & 4.099405000 \\
\hline $\mathrm{C}$ & 10.269038000 & 6.788211000 & 3.297048000 \\
\hline $\mathrm{H}$ & 10.340247000 & 6.578095000 & 2.237928000 \\
\hline $\mathrm{C}$ & 11.246835000 & 7.426961000 & 4.131672000 \\
\hline $\mathrm{H}$ & 12.220348000 & 7.796684000 & 3.839677000 \\
\hline $\mathrm{C}$ & 10.707805000 & 7.460389000 & 5.381615000 \\
\hline $\mathrm{H}$ & 11.059652000 & 7.816063000 & 6.338424000 \\
\hline $\mathrm{C}$ & 6.632149000 & 9.986602000 & 2.198781000 \\
\hline $\mathrm{C}$ & 7.422759000 & 11.450915000 & 0.732643000 \\
\hline $\mathrm{H}$ & 8.077870000 & 11.894517000 & -0.002772000 \\
\hline $\mathrm{C}$ & 4.538709000 & 11.317591000 & 2.687216000 \\
\hline $\mathrm{H}$ & 4.577794000 & 12.312075000 & 3.142598000 \\
\hline $\mathrm{H}$ & 4.426316000 & 10.575627000 & 3.478577000 \\
\hline $\mathrm{C}$ & 3.397994000 & 11.224180000 & 1.675244000 \\
\hline $\mathrm{H}$ & 3.541575000 & 11.945689000 & 0.865882000 \\
\hline
\end{tabular}




$\begin{array}{llll}\mathrm{H} & 2.447701000 & 11.445292000 & 2.173083000 \\ \mathrm{H} & 3.341690000 & 10.218592000 & 1.247435000 \\ \mathrm{C} & 8.695546000 & 9.257829000 & 1.029789000 \\ \mathrm{H} & 9.361674000 & 9.150110000 & 1.891690000 \\ \mathrm{H} & 9.269785000 & 9.611861000 & 0.173197000 \\ \mathrm{C} & 8.069985000 & 7.892469000 & 0.654136000 \\ \mathrm{C} & 6.272214000 & 6.361655000 & 0.946586000 \\ \mathrm{H} & 6.515823000 & 5.463866000 & 1.526507000 \\ \mathrm{H} & 6.572265000 & 6.161935000 & -0.089381000 \\ \mathrm{C} & 4.792083000 & 6.539259000 & 1.033098000 \\ \mathrm{C} & 3.821002000 & 5.890757000 & 1.743768000 \\ \mathrm{H} & 3.988915000 & 5.096266000 & 2.458918000 \\ \mathrm{C} & 2.566009000 & 6.468883000 & 1.354736000 \\ \mathrm{H} & 1.579625000 & 6.195865000 & 1.704323000 \\ \mathrm{C} & 2.864297000 & 7.427284000 & 0.434770000 \\ \mathrm{H} & 2.269412000 & 8.109070000 & -0.154300000\end{array}$

Table S8. B3LYP/6-31G*, LANL2DZ level optimized coordinates of $\mathbf{2 b}$.

Ground state electronic energy $=-1840.7924673$ Hartree/Particle.

$\begin{array}{lrrr}\mathrm{Ni} & 7.191189000 & 6.791227000 & 0.133428000 \\ \mathrm{O} & 9.778939000 & 6.752433000 & 3.335727000 \\ \mathrm{O} & 7.591890000 & 3.170954000 & 1.629734000 \\ \mathrm{O} & 8.722956000 & 5.192387000 & -3.337491000 \\ \mathrm{O} & 9.751144000 & 9.242077000 & -1.571140000 \\ \mathrm{~N} & 4.843618000 & 6.914459000 & 2.045013000 \\ \mathrm{~N} & 6.601885000 & 7.928565000 & 2.676696000 \\ \mathrm{~N} & 4.562559000 & 7.371995000 & 3.312788000 \\ \mathrm{~N} & 8.525208000 & 6.274214000 & 1.446882000 \\ \mathrm{~N} & 5.188960000 & 8.378965000 & -1.493813000 \\ \mathrm{~N} & 5.699992000 & 6.466886000 & -2.269050000 \\ \mathrm{~N} & 4.529519000 & 8.288471000 & -2.699048000 \\ \mathrm{~N} & 8.356768000 & 6.319543000 & -1.346255000 \\ \mathrm{C} & 6.087532000 & 7.221083000 & 1.630515000 \\ \mathrm{C} & 5.658365000 & 7.983714000 & 3.667555000 \\ \mathrm{H} & 5.808442000 & 8.476601000 & 4.616970000 \\ \mathrm{C} & 3.881011000 & 6.004031000 & 1.392032000 \\ \mathrm{H} & 4.229817000 & 5.934938000 & 0.358728000 \\ \mathrm{C} & 3.957017000 & 4.619505000 & 2.046614000 \\ \mathrm{H} & 4.971858000 & 4.214169000 & 1.990519000 \\ \mathrm{H} & 3.277113000 & 3.930625000 & 1.533431000 \\ \mathrm{H} & 3.660601000 & 4.676724000 & 3.098977000 \\ \mathrm{C} & 2.470572000 & 6.595111000 & 1.424505000 \\ \mathrm{H} & 2.121117000 & 6.710644000 & 2.454063000 \\ \mathrm{H} & 1.783905000 & 5.925114000 & 0.897096000 \\ \mathrm{H} & 2.436387000 & 7.573841000 & 0.935684000 \\ \mathrm{C} & 8.025239000 & 8.270401000 & 2.752485000 \\ \mathrm{H} & 8.240893000 & 8.654284000 & 3.749623000\end{array}$




$\begin{array}{cccc}\mathrm{H} & 8.250665000 & 9.041359000 & 2.008868000 \\ \mathrm{C} & 8.876309000 & 6.995774000 & 2.524382000 \\ \mathrm{C} & 9.219404000 & 4.979835000 & 1.332338000 \\ \mathrm{H} & 10.263894000 & 5.104097000 & 1.636727000 \\ \mathrm{H} & 9.189149000 & 4.668662000 & 0.286522000 \\ \mathrm{C} & 8.612061000 & 3.909535000 & 2.182978000 \\ \mathrm{C} & 8.834244000 & 3.490318000 & 3.462579000 \\ \mathrm{H} & 9.569762000 & 3.907952000 & 4.134810000 \\ \mathrm{C} & 7.904716000 & 2.428537000 & 3.719571000 \\ \mathrm{H} & 7.799926000 & 1.852604000 & 4.629040000 \\ \mathrm{C} & 7.179732000 & 2.276244000 & 2.577880000 \\ \mathrm{H} & 6.390546000 & 1.603758000 & 2.276935000 \\ \mathrm{C} & 5.928822000 & 7.291465000 & -1.207079000 \\ \mathrm{C} & 4.866546000 & 7.110004000 & -3.144674000 \\ \mathrm{H} & 4.532061000 & 6.686893000 & -4.080609000 \\ \mathrm{C} & 5.180812000 & 9.670583000 & -0.777599000 \\ \mathrm{H} & 5.603752000 & 9.440020000 & 0.203376000 \\ \mathrm{C} & 3.750729000 & 10.189352000 & -0.616973000 \\ \mathrm{H} & 3.297064000 & 10.387162000 & -1.591967000 \\ \mathrm{H} & 3.764623000 & 11.122222000 & -0.044156000 \\ \mathrm{H} & 3.122771000 & 9.469142000 & -0.083068000 \\ \mathrm{C} & 6.094221000 & 10.666168000 & -1.501992000 \\ \mathrm{H} & 7.112196000 & 10.273647000 & -1.586016000 \\ \mathrm{H} & 6.128402000 & 11.609047000 & -0.945165000 \\ \mathrm{H} & 5.714174000 & 10.872896000 & -2.507725000 \\ \mathrm{C} & 6.488325000 & 5.250290000 & -2.485720000 \\ \mathrm{H} & 6.266877000 & 4.860837000 & -3.479414000 \\ \mathrm{H} & 6.214531000 & 4.504804000 & -1.732412000 \\ \mathrm{C} & 7.997366000 & 5.595049000 & -2.419114000 \\ \mathrm{C} & 9.750132000 & 6.797186000 & -1.375174000 \\ \mathrm{H} & 10.390196000 & 6.013036000 & -1.792857000 \\ \mathrm{H} & 10.062167000 & 7.000509000 & -0.349071000 \\ \mathrm{C} & 9.932431000 & 8.032012000 & -2.200205000 \\ \mathrm{C} & 10.231051000 & 8.243772000 & -3.514890000 \\ \mathrm{H} & 10.407620000 & 7.467196000 & -4.245101000 \\ \mathrm{C} & 10.241840000 & 9.663999000 & -3.715480000 \\ \mathrm{H} & 10.450691000 & 10.196569000 & -4.633387000 \\ \mathrm{C} & 9.947014000 & 10.218446000 & -2.508048000 \\ \mathrm{H} & 9.860388000 & 11.234133000 & -2.152397000\end{array}$

Table S9. B3LYP/6-31G*, LANL2DZ level optimized coordinates of $\mathbf{3 b}$.

Ground state electronic energy $=-2145.6187651$ Hartree/Particle.

$\begin{array}{lllc}\mathrm{Ni} & -0.217899000 & 0.576260000 & 12.884264000 \\ \mathrm{C} & -2.184900000 & 2.082601000 & 15.974939000 \\ \mathrm{H} & -3.050806000 & 2.463308000 & 15.450058000 \\ \mathrm{O} & -1.822302000 & 1.661775000 & 9.367438000 \\ \mathrm{O} & -4.189654000 & 1.562038000 & 12.866267000\end{array}$




\begin{tabular}{|c|c|c|c|}
\hline $\mathrm{O}$ & 1.940354000 & 4.030750000 & 13.147252000 \\
\hline $\mathrm{N}$ & 2.117903000 & -1.150443000 & 13.830172000 \\
\hline $\mathrm{N}$ & 3.492842000 & -1.139701000 & 13.759141000 \\
\hline $\mathrm{N}$ & 2.600529000 & 0.604381000 & 12.737719000 \\
\hline $\mathrm{N}$ & 0.490937000 & 2.263223000 & 13.537248000 \\
\hline $\mathrm{N}$ & -1.920366000 & 1.378145000 & 12.409318000 \\
\hline $\mathrm{N}$ & -2.258878000 & -1.383112000 & 12.783760000 \\
\hline $\mathrm{N}$ & -1.654299000 & -3.118562000 & 11.559953000 \\
\hline $\mathrm{N}$ & -0.667106000 & -2.163631000 & 11.616757000 \\
\hline $\mathrm{C}$ & 1.536834000 & -0.101161000 & 13.216363000 \\
\hline $\mathrm{C}$ & 3.751180000 & -0.050474000 & 13.090721000 \\
\hline $\mathrm{H}$ & 4.743274000 & 0.297162000 & 12.842166000 \\
\hline $\mathrm{C}$ & 1.465657000 & -2.217412000 & 14.593396000 \\
\hline $\mathrm{H}$ & 1.644684000 & -3.166693000 & 14.077921000 \\
\hline $\mathrm{H}$ & 0.395615000 & -2.000128000 & 14.545395000 \\
\hline $\mathrm{C}$ & 1.951370000 & -2.303925000 & 16.027015000 \\
\hline $\mathrm{C}$ & 2.303854000 & -3.545135000 & 16.564720000 \\
\hline $\mathrm{H}$ & 2.261217000 & -4.434947000 & 15.940492000 \\
\hline $\mathrm{C}$ & 2.715994000 & -3.651375000 & 17.895040000 \\
\hline $\mathrm{H}$ & 2.987786000 & -4.622369000 & 18.299892000 \\
\hline $\mathrm{C}$ & 2.790920000 & -2.510752000 & 18.694351000 \\
\hline $\mathrm{H}$ & 3.120140000 & -2.588383000 & 19.726941000 \\
\hline $\mathrm{C}$ & 2.447354000 & -1.265930000 & 18.159110000 \\
\hline $\mathrm{H}$ & 2.512567000 & -0.372255000 & 18.774238000 \\
\hline $\mathrm{C}$ & 2.024338000 & -1.160920000 & 16.834219000 \\
\hline $\mathrm{H}$ & 1.754109000 & -0.189608000 & 16.429140000 \\
\hline $\mathrm{C}$ & -2.170349000 & 1.270010000 & 17.158440000 \\
\hline $\mathrm{H}$ & -3.021652000 & 0.930145000 & 17.732392000 \\
\hline $\mathrm{C}$ & -0.859912000 & 1.018974000 & 17.425873000 \\
\hline $\mathrm{H}$ & -0.347245000 & 0.458742000 & 18.192766000 \\
\hline $\mathrm{O}$ & -0.060229000 & 1.620851000 & 16.495953000 \\
\hline $\mathrm{C}$ & -0.883175000 & 2.268015000 & 15.605128000 \\
\hline $\mathrm{C}$ & -0.248237000 & 3.067504000 & 14.516407000 \\
\hline $\mathrm{H}$ & 0.449021000 & 3.800602000 & 14.941073000 \\
\hline $\mathrm{H}$ & -1.050691000 & 3.648075000 & 14.043417000 \\
\hline $\mathrm{C}$ & 1.575123000 & 2.855164000 & 13.012936000 \\
\hline $\mathrm{C}$ & 2.457308000 & 1.934033000 & 12.135543000 \\
\hline $\mathrm{H}$ & 2.027385000 & 1.834004000 & 11.133671000 \\
\hline $\mathrm{H}$ & 3.445527000 & 2.388245000 & 12.058419000 \\
\hline $\mathrm{C}$ & -0.998642000 & -1.086175000 & 12.356482000 \\
\hline $\mathrm{C}$ & -2.613698000 & -2.604699000 & 12.278610000 \\
\hline $\mathrm{H}$ & -3.568282000 & -3.077153000 & 12.458324000 \\
\hline $\mathrm{C}$ & 0.577069000 & -2.409105000 & 10.871045000 \\
\hline $\mathrm{H}$ & 1.044209000 & -3.299629000 & 11.304153000 \\
\hline $\mathrm{H}$ & 1.217400000 & -1.550058000 & 11.070039000 \\
\hline $\mathrm{C}$ & 0.366781000 & -2.593583000 & 9.383440000 \\
\hline $\mathrm{C}$ & 0.424154000 & -1.493824000 & 8.519359000 \\
\hline $\mathrm{H}$ & 0.601983000 & -0.497023000 & 8.915339000 \\
\hline $\mathrm{C}$ & 0.248152000 & -1.667461000 & 7.145737000 \\
\hline $\mathrm{H}$ & 0.301447000 & -0.807106000 & 6.484283000 \\
\hline
\end{tabular}




$\begin{array}{lrrr}\mathrm{C} & 0.012325000 & -2.941071000 & 6.624497000 \\ \mathrm{H} & -0.121023000 & -3.076160000 & 5.554543000 \\ \mathrm{C} & -0.048140000 & -4.041837000 & 7.481851000 \\ \mathrm{H} & -0.230779000 & -5.035539000 & 7.082134000 \\ \mathrm{C} & 0.130522000 & -3.868328000 & 8.854475000 \\ \mathrm{H} & 0.079793000 & -4.724707000 & 9.521398000 \\ \mathrm{C} & -1.083770000 & 1.772766000 & 8.226447000 \\ \mathrm{H} & -1.415130000 & 1.168497000 & 7.395775000 \\ \mathrm{C} & -0.065613000 & 2.660021000 & 8.402958000 \\ \mathrm{H} & 0.668993000 & 2.954415000 & 7.665734000 \\ \mathrm{C} & -0.185204000 & 3.133237000 & 9.753740000 \\ \mathrm{H} & 0.435684000 & 3.864411000 & 10.254558000 \\ \mathrm{C} & -1.267161000 & 2.499234000 & 10.299193000 \\ \mathrm{C} & -1.950482000 & 2.614405000 & 11.621896000 \\ \mathrm{H} & -1.466073000 & 3.439391000 & 12.153845000 \\ \mathrm{H} & -3.001882000 & 2.891014000 & 11.478791000 \\ \mathrm{C} & -3.106464000 & 0.961062000 & 12.879596000 \\ \mathrm{C} & -3.093446000 & -0.439028000 & 13.533284000 \\ \mathrm{H} & -2.729825000 & -0.371365000 & 14.563579000 \\ \mathrm{H} & -4.117117000 & -0.814297000 & 13.539432000\end{array}$




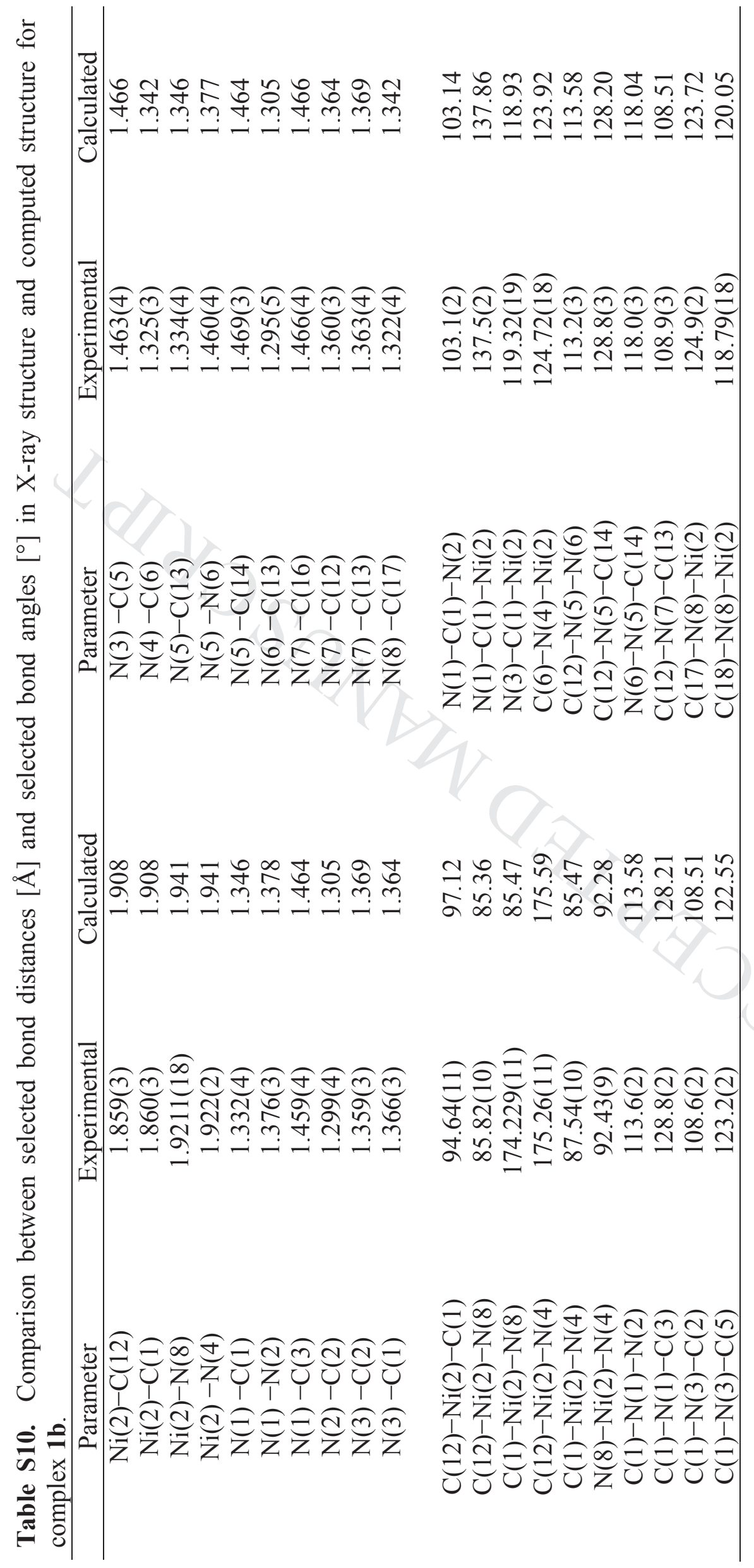




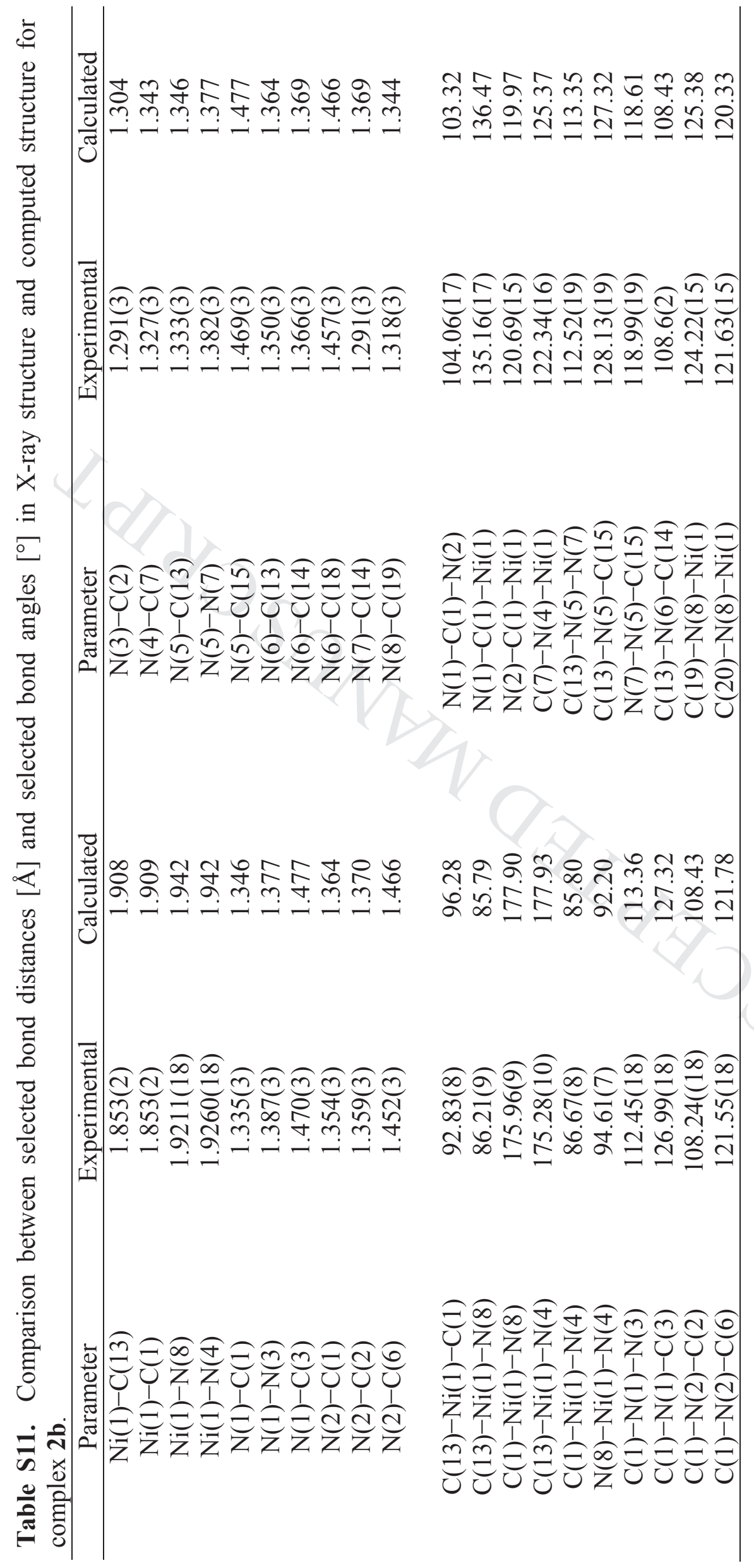




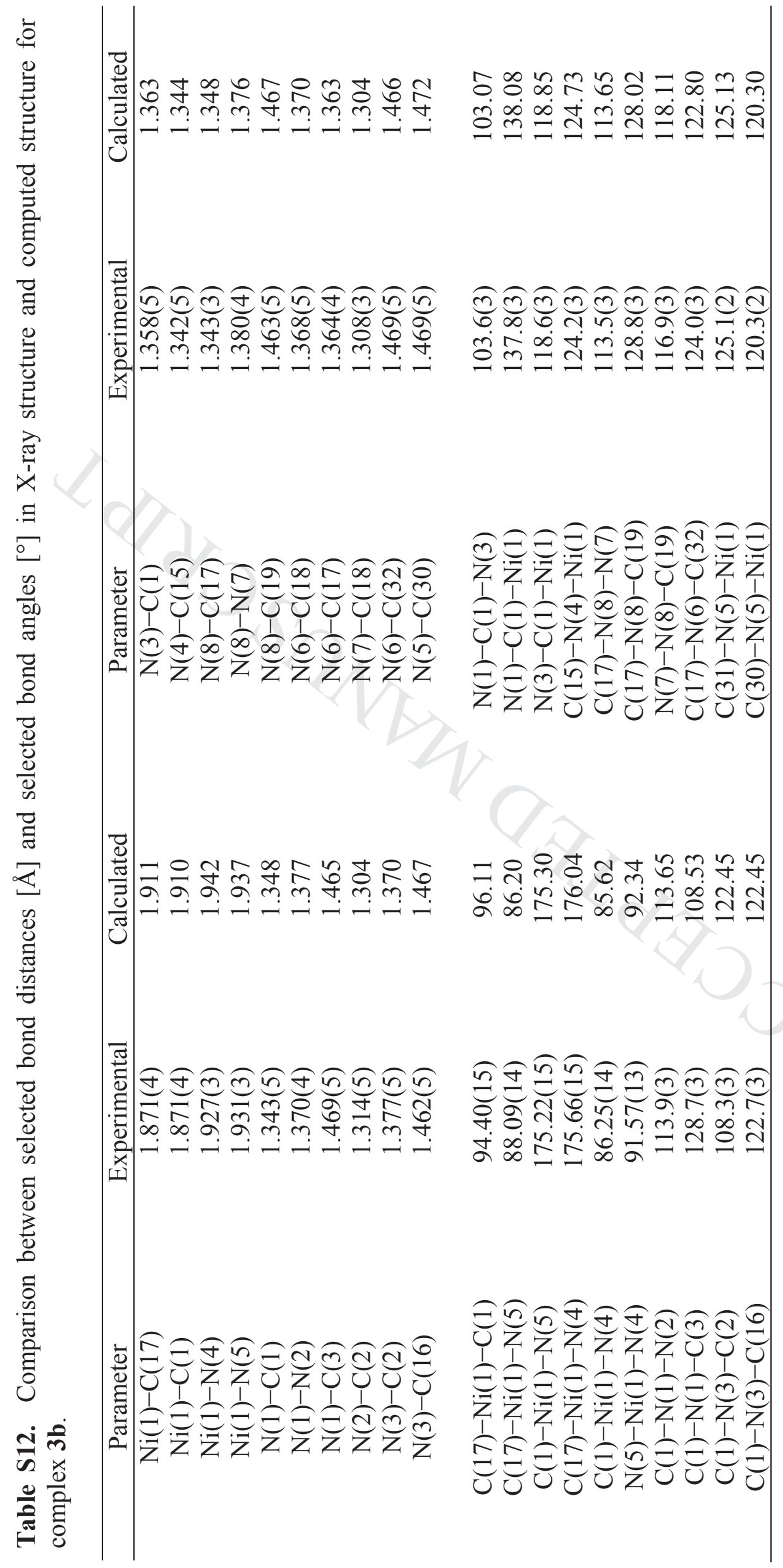

